\title{
Polymeric Nanostructured Materials for Biomedical Applications
}

\author{
Zhaohui Tang ${ }^{\mathrm{a}}$, Chaoliang He ${ }^{\mathrm{a}}$, Huayu Tian ${ }^{\mathrm{a}}$, Jianxun Ding ${ }^{\mathrm{a}}$, \\ Benjamin S. Hsiao ${ }^{\mathrm{b}, *}$, Benjamin $\mathrm{Chu}^{\mathrm{b}}$ and Xuesi Chen ${ }^{\mathrm{a}, *}$ \\ ${ }^{a}$ Key Laboratory of Polymer Ecomaterials, Changchun Institute of Applied Chemistry, Chinese \\ Academy of Sciences, Changchun 130022, China \\ ${ }^{\mathrm{b}}$ Department of Chemistry, Stony Brook University, Stony Brook, NY 11794-3400, United States
}

\begin{abstract}
Polymeric nanostructured materials (PNMs), which are polymeric materials in nanoscale or polymer composites containing nanomaterials, have become increasingly useful for biomedical applications. In specific, advances in polymer-related nanoscience and nanotechnology have brought a revolutionary change to produce new biomaterials with tailored properties and functionalities for targeted biomedical applications. These materials, including micelles, polymersomes, nanoparticles, nanocapsules, nanogels, nanofibers, dendrimers and nanocomposites, have been widely used in drug delivery, gene therapy, bioimage, tissue engineering and regenerative medicine. This review presents a comprehensive overview on the various types of PNMs, their fabrication methods and biomedical applications, as well as the challenges in research and development of future PNMs.
\end{abstract}

Keywords: Polymer, Nanostructure, Biomaterials, Nanocarriers, Biomedical Applications

* Corresponding authors: xschen@ciac.ac.cn (XS. Chen), Tel.: +86 431 85262112, Fax: +86 431 85262112

benjamin.hsiao@stonybrook.edu (BS. Hsiao), Tel.: +1 631632 7793, Fax: +16316326518 


\section{Contents}

1. Introduction

2. Fabrication of polymeric nanostructured materials

2.1 Micelles

2.2 Polymersomes

2.3 Nanoparticles

2.4 Nanocapsules formed on sacrificial templates

2.5 Nanogels

2.6 Nanofibers

2.7 Others

3. Biomedical applications of polymeric nanostructured materials

3.1 Drug delivery

3.2 Gene carriers

3.3 Bioimaging

3.4 Tissue engineering and regenerative medicine

4. Conclusions and future challenges

\section{References}




\section{List of abbreviations}

3D

ACA

AETMAC

ATRP

AuNPs

bFGF

BM-MSCs

CD44

CPA

CPT

CR-CAs

CS

CT

CTA

DEXlactateHEMA

DEX-PCL

DexS

DLC

DLE

DMIPM

DNA

DOTMA

DOX

DTT

ECMs

EGDMA

EPR effect

FDA

FDG

FI three-dimensional

alkylcyanoacrylates

2-acryloxyethyltrimethylammonium chloride

atom transfer radical polymerization

gold nanoparticles

basic fibroblast growth factor

bone marrow-derived mesenchymal stem cells

cluster determinant 44

collagen-mimetic peptide amphiphile

camptothecin

cancer-recognizable MRI contrast agents

chitosan

X-ray computed tomography

chain transfer agent

dextran-lactate-2-hydroxyethyl methacrylate

dextran-block-poly ( $\varepsilon$-caprolactone)

dextran sulfate

drug loading capacity

drug loading efficiency

2,3-dimethylmaleic imidopropyl methacrylate

deoxyribonucleic acid

$\mathrm{N}$-[1-(2,3,-dioleyloxy)propyl]-N,N,N-trimethylammonium chloride doxorubicin

1,4-dithio-DL-threitol

extracellular matrices

ethylene glycol dimethacrylate

enhanced permeation retention effect

US Food and Drug Administration

fluorodeoxyglucose

fluorescence imaging 
FITC

GA

GSH

HEA

HF

HPMA

LA

LbL

LCST

L-Cys NCA

LMW

MBA

MMA

MMC

mPEG-PDLLA

MRI

MTX

NIPAM

NK

O/W

OEG-A

PAA

PACA

PAMAM

PCL

PDLA

PDLLA

PEG

PEG- $b$-PCL

PEG- $b$-PLA

PEG- $b$-PLGA

PEGDA fluorescein isothiocyanate

glutaraldehyde or glycolide

glutathione

2-hydroxyethylacrylate

hydrofluoric acid

$N$-(2-hydroxypropyl) methacrylamide

lactide

layer-by-layer

lower critical solution temperature

L-cystine $N$-carboxyanhydride

low molecular weight

$\mathrm{N}, \mathrm{N}^{\prime}$-methylenebisacylamide

methyl methacrylate

mitomycin $\mathrm{C}$

monomethoxy poly(ethylene glycol)-block-poly(D,L-lactide)

magnetic resonance imaging

methotrexate

$\mathrm{N}$-isopropylacrylamide

natural killer

oil-in-water

oligoethylene glycol acrylate

poly(L-aspartic acid)

poly(alkylcyanoacrylate)

polyamidoamine

$\operatorname{poly}(\varepsilon$-caprolactone $)$

poly(D-lactide)

poly(D,L-lactide)

poly(ethylene glycol)

poly(ethylene glycol)- $b$-poly( $\varepsilon$-caprolactone)

poly(ethylene glycol)- $b$-polylactide

poly(ethylene glycol)- $b$-poly(lactide-co-glycolide)

poly(ethylene glycol) diacrylate 
PEG-p(L-His)

methoxy poly(ethylene glycol)- $b$-poly(L-histidine)

PEG-p(L-LA)-DTPA-Gd methoxy poly(ethylene glycol)-b-poly(L-lactic

acid)-diethylenetriaminopentaacetic acid dianhydride-gadolinium chelate

PEO-PAA-PNIPAM

PEG-PAC

PEG-PCL

PEG-PCL-PEG

PEG-PUC

PEI

PEO

PEO-PBLA

PET

PGA

PiPrOx-P(Asp)

PiPrOx-P(Lys)

PK1

PLA

PLGA

PLL

PLLA

PMMA

PMPA

POEGMA

$\operatorname{poly}(\mathrm{S}-\mathrm{co}-\mathrm{MAA})$

PTX

PU

PVA

PVAc

QDs poly(ethylene oxide)- $b$-poly(acrylic

acid)- $b$-poly(N-isopropylacrylamide)

acid-functionalized poly(carbonate) and poly(ethylene glycol) diblock copolymer

poly(ethylene glycol)-block-poly(e-caprolactone)

poly(ethylene glycol)-poly(e-caprolactone)-poly(ethylene glycol)

urea-functionalized poly(carbonate) and poly(ethylene glycol) diblock

copolymer

polyethylenimine

poly(ethylene oxide)

poly(ethylene oxide)-poly( $\beta$-benzyl L-aspartate $)$

positron emission tomography

poly(L-glutamic acid)

poly(2-isopropyl-2-oxazoline)- $b$-poly(aspartic acid)

poly(2-isopropyl-2-oxazoline)- $b$-poly(L-lysine)

HPMA copolymer-DOX conjugate

poly(lactic acid)

poly(DL-lactide-co-glycolide)

poly(L-lysine)

poly(L-lactide)

poly(methyl methacrylamide)

poly[(3-morpholinopropyl) aspartamide]

poly(oligo(ethylene glycol) methacrylate)

poly(styrene-co-methacrylic acid)

paclitaxel

polyurethane

poly(vinyl alcohol)

Poly (vinyl acetate)

quantum dots 
RAFT

RGD

RHAMM

RNA

ROP

$\mathrm{S} / \mathrm{O} / \mathrm{O}$

SCK

SI-ATRP

siRNA

SPECT

SPIO

tBMA

TPT

USPIO

VEGF

W/O

W/O/O

W/O/W

$\beta-C D-S$ reversible addition-fragmentation chain transfer

Arg-Gly-Asp

receptor for hyaluronate-mediated motility

ribonucleic acid

ring-opening polymerization

solid-in-oil-in-oil

shell crosslinked

surface initiated atom transfer radical polymerization

short interfering RNA

single-photo emission computed tomography

super paramagnetic iron oxide

tert-butyl methacrylate

tetraaniline-polylactide-tetraaniline

ultra-small super paramagnetic iron oxide

vascular endothelial growth factor

water-in-oil

water-in-oil-in-oil

water-in-oil-in-water

thiol- $\beta$-cyclodextrin 


\section{Introduction}

Polymeric nanostructured materials (PNMs) have been playing an increasingly important role to revolutionize the diagnoses and treatments of diseases [1-3]. Through the development of PNMs as new biomaterials, significant improvement in the quality of health care be achieved, due to the better accuracy and reliability in diagnostics, more effective targeting of therapeutic agents, and improved usability of scaffolds for tissue engineering and regenerative medicine, just to name a few [1-16].

PNMs (Fig. 1), including micelles, polymersomes, nanoparticles, nanocapsules, nanogels, nanofibers, dendrimers, brush polymers and nanocomposites, can be prepared in a variety of pathways. Their properties, such as stability, size, shape, surface charge, surface chemistry, mechanical strength, porosity, and so on, can be tailored towards the specific functionalities that are required to meet the needs of the targeted biomedical application. As a result, the development of biomedical PNMs has attracted a great deal of research interests in the field, where a very large number of recent publications can be found in the literature [17-26]. In general, PNMs for biomedical applications should have: a) significant water solubility or dispersibility, b) well-controlled nanoparticle dimension to avoid fast clearance (10-200 nm) and to achieve preferred biodistribution, c) biodegradability to minimize side effects (residue with $\mathrm{MW}<45 \mathrm{k}$ or hydrodynamic size $<10 \mathrm{~nm}$ for complete clearance from circulation), d) functionality to link with prodrug, targeting component, or imaging element, etc. e) responsivity to release therapeutic loading 
under triggered conditions.

Fig. 1.

One unique property of PNMs, in particular those intended for nanocarrier usage such as contrast agents or therapeutic agents in cancer diagnosis or cancer therapy, is related to their prolonged blood circulation time, which allows the agent to have more opportunity to accumulate in the tumor cells via either the "passively" or "actively" route [5, 27-29]. This is in contrast with small molecules agents or drugs that often extravasate from vascular structures rapidly, where more than half of the injected dose can be gone from the blood circulation in just one or two passes of the cardiac cycle. Thus, for bioimaging applications, the contrasting agents based on larger polymeric nanoparticles not only could remain in the vasculature to give higher angiography contrast, but the tumor assessment could also be less hindered by the rapidly changing arterial input function [30]. For therapeutic applications, PNMs also offer better efficacy and lower systemic toxicity because of the enhanced targeting and sustained release ability of polymeric nano-drugs $[8,31,32]$. For these reasons and other considerations such as high tunability, good biocompatibility and diversity, PNMs shall play a more vital role in the future for biomedical applications, especially for the development of new controlled-released drugs.

Due to the increasing interests and great potentials of PNMs for biomedical applications, this review is intended to give an overview of the current state-of-the-art fabrication methods to produce 
PNMs and their intended biomedical applications. The challenges and outlook of developing new

PNMs for future applications will be also discussed.

\section{Fabrication of polymeric nanostructured materials}

\subsection{Micelles}

Polymeric micelle is a sub-microscopic aggregate of polymeric molecules dispersed in liquid, forming a colloidal system. It typically has a core-shell architecture, where the inner core is composed of the hydrophobic part of the amphiphiles, and the core region, surrounded by a corona, is composed of the hydrophilic part of the amphiphiles [33]. The micelle formation is a process of the force balance between the attractive and repulsive interactions. There are different types of attractive forces, which govern the segregation of the core segment from the aqueous phase. The forces include hydrophobic interactions, electrostatic interactions, and complexation [34]. The repulsive forces can be caused by different sources such as electrostatic repulsion, hydration, and steric interactions. These forces prevent the unlimited growth of the micelles $[35,36]$. Similar to low molecular weight surfactants, amphiphilic copolymers also possess a critical micelle concentration $(\mathrm{CMC})$ in the process of micelle formation. At concentrations below $\mathrm{CMC}$, the copolymers only exist as individual molecules in solution. When the concentration is above CMC, the amphiphilic copolymer chains can associate and form micelles in a way that the hydrophobic part of the copolymer would avoid direct contact with the aqueous media [35]. Polymeric micelles are generally 
composed of several hundred molecules, where the corresponding diameter typically ranges from 10 to $100 \mathrm{~nm}$ [34]. The micellization process is entropy-driven and has an endothermic enthalpy [37, 38]. Based on light scattering, sedimentation velocity and small-angle $\mathrm{X}$-ray scattering studies, the aggregated polymeric molecules in the micelle are in a dynamic equilibrium with the unimers in the bulk phase [33, 39-41]. The fabrication methods to prepare polymeric micelle can be classified into four categories: direct dissolution, dialysis, film casting and oil-in-water $(\mathrm{O} / \mathrm{W})$ emulsion, which are described below. The selection of the proper method must consider the solubility of the polymers in water. Some representative examples of polymeric micelles and their compositions are illustrated in Table 1.

Table 1.

\subsubsection{Direct dissolution}

The direct dissolution method is usually employed to prepare micelles from block or graft copolymers with moderate solubility in water. The method simply involves the addition of copolymers to water or other aqueous medium such as phosphate buffer saline without using any organic solvents and surfactants. In some cases, copolymer and water are mixed at elevated temperatures to promote micellization $[42,58]$. The direct dissolution method is routinely used for the preparation of Pluronic (Block copolymer of ethylene oxide and propylene oxide) micelles [43].

It can also be used for the fabrication of micelles from poly(ethylene glycol)-polyester copolymers. 
For example, Qian et al. demonstrated the preparation of blank micelles by directly dissolving poly(ethylene glycol)-poly(e-caprolactone)-poly(ethylene glycol) (PEG-PCL-PEG) in distilled water at $50{ }^{\circ} \mathrm{C}$. The drug-loaded micelles were subsequently prepared by this method assisted by ultrasonication [58].

The direct dissolution method can also be used to fabricate micelles formed by electrostatic interactions or metal complexation. Kataoka et al. reported the preparation of polyion complex micelles by direct mixing the solutions of poly(2-isopropyl-2-oxazoline)- $b$-poly(L-lysine) [PiPrOx-P(Lys)] and poly(2-isopropyl-2-oxazoline)-b-poly(aspartic acid) [PiPrOx-P(Asp)] in a Tris-HCl buffered solution [44]. Similarly, doxorubicin-loaded micelles could be prepared by direct mixing the aqueous solutions of doxorubicin and carboxyl-containing polymers, where the preparation scheme is illustrated in Fig. 2 [45, 59]. Polymeric micelles containing platinum-based anticancer drugs were also demonstrated through the polymer-metal complex formation by mixing the aqueous solution of polypeptide and drug [46, 60-68].

Fig. 2.

Although the direct dissolution method is simple, it requires that the hydrophobic segments can be swollen quite extensively in water and reach the equilibrium within a reasonable time period during micelle preparation. However, as most polymeric micelles form very rapidly in water and become kinetically 'frozen' once they reach certain size $[69,70]$, the direct dissolution method 
sometimes becomes inapplicable for the preparation of polymeric micelles with desired structure and properties, therefore, other methods are necessary.

\subsubsection{Film casting}

The film casting method is often referred to as the dry-down method or the solution casting method, which involves the dissolution of copolymer (or copolymer and drug mixtures) in a volatile organic solvent. In this method, the solvent is first evaporated to create a thin film. Subsequently, a warm buffer solution or water is added under agitation to dissolve the polymer film. The hydrophobic part forms the core of the micelle, while the hydrophilic block becomes hydrated and dissolves to form the shell (corona) [33, 71]. Allen et al. reported the micelle preparation scheme by hydrating the amphiphilic copolymer film in a phosphate buffer saline at $60^{\circ} \mathrm{C}[47,72]$. With this method, cancer drug loaded micelles could be prepared by the hydration of paclitaxel containing monomethoxy poly(ethylene glycol)-block-poly(D,L-lactide) (mPEG-PDLLA) films in water [48].

Similarly, thioridazine-loaded micelles were prepared by the hydration of thioridazine containing lipid film based on acid-functionalized poly(carbonate) and poly(ethylene glycol) diblock copolymer (PEG-PAC) and urea-functionalized poly(carbonate) and poly(ethylene glycol) diblock copolymer (PEG-PUC) with phosphate buffered saline (PBS 7.4) at $50{ }^{\circ} \mathrm{C}[49]$.

\subsubsection{Dialysis}


The dialysis method can be utilized for micelle preparation if the copolymer has low solubility in water. In this method, the micelle-forming copolymer is first dissolved in a water miscible organic solvent, such as ethanol [57], acetone [73], dimethylsulfoxide [53, 55], dimethylformamide [53, 54], $\mathrm{N}, \mathrm{N}$-dimethylacetamide [49, 53], tetrahydrofuran [53], acetonitrile [52], where the dialysis is carried out subsequently against the aqueous media to remove the solvent. The solvent selection in this method can dramatically affect the stability of the polymeric micelles [51]. For example, using dimethylsulfoxide as the initial solvent to dissolve poly(ethylene oxide)-poly( $\beta$-benzyl L-aspartate) (PEO-PBLA) and followed by the dialysis against water, only $6 \mathrm{wt} \%$ of the total amount of PEO-PBLA could form micelles, where a considerable amount of secondary aggregates was also present. However, when N,N-dimethylacetamide was used as the initial solvent, the micelles were obtained in a very high yield (87 wt.\%) [51]. Compared to the direct dialysis of polymer solution in the dialysis bag against water, the rapid addition of the organic solution in water and vice versa prior to the dialysis can produce micelles with a more reproducible and narrower size-distribution [53].

\subsubsection{Oil-in-water emulsion}

The oil-in-water $(\mathrm{O} / \mathrm{W})$ emulsion method is usually used for the preparation of drug-loaded micelles, involving the dissolution of the drug and copolymer in a volatile, non-water-miscible organic solvent, such as dichloromethane [56, 57, 74], ethyl acetate $[4,75]$ and chloroform [76], first. The $\mathrm{O} / \mathrm{W}$ emulsion is subsequently formed in an aqueous medium by vortexing and sonicating, 
which is followed by the evaporation of the organ solvent. The $\mathrm{O} / \mathrm{W}$ emulsion method has an advantage over the dialysis method, where the drug is present in the inner organic droplets of the emulsion that are stabilized by the copolymer. Upon evaporation of the organic solvent, the drug will remain in the core of the micelles, thereby increasing the drug loading capacity. This is contrasted to the dialysis method, in which the drug may precipitate before being incorporated in the micelles if the solvent diffuses rapidly from micelle core, or if the drug molecules interact with each other more favorably than with the copolymer. Sant et al. observed at least 1.5 -fold rise in the incorporation efficiency of drug loading by the O/W emulsion method, when compared to the dialysis method [57].

\subsection{Polymersomes}

Polymersomes (also referred to as polymer vesicles) are a class of artificial vesicles made from amphiphilic polymers. Similar to liposomes, typical polymersomes are hollow assemblies that contain an aqueous solution in the core surrounded by a bi-layer membrane shell. However, as a result of their significantly higher molecular weight, polymersomes exhibit enhanced mechanical stability and reduced permeability, possessing an impermeable physical barrier capable of isolating the encapsulated functional materials from the environment. The bi-layer membrane is composed of hydrated hydrophilic coronas, located at both the inside and outside of the hydrophobic part of the membrane. The aqueous core can be utilized to encapsulate therapeutic molecules, such as low molecular weight drugs, proteins and deoxyribonucleic acid (DNA) or ribonucleic acid (RNA) 
fragments. Therefore, polymersomes have attracted a great deal of research interests recently and have been highlighted for a number of biomedical applications [77-84].

Similar to micelles, hydrophobic interactions are usually the mainly driving force in the polymersome formation. Many techniques can be used to prepare the polymersomes by self-assemble of amphiphilic block copolymers, and the recent advancement in polymer synthesis has made it possible to prepare small to giant polymersomes spanning from nano- to microscales [85], including stimuli-sensitive polymersomes that can respond to an external or internal stimulus $[19,86,87]$, chimaeric polymersomes that contain distinct interior environments separated from the outside by an asymmetric membrane [88], porous polymersomes with tailored permeability [89], and biomimetic and targeting polymersomes that selectively deliver drugs, proteins, and/or imaging probes to the action sites $[90,91]$. The most important preparation methods to prepare polymersome are generally classified into two groups: the organic solvent based method (by the solvent-switch technique) and the solvent free method (by the polymer rehydration technique). In the following sub-section, these two methods were discussed in detail.

\subsubsection{Organic solvent-based method}

In the organic solvent-based method, polymersomes are formed by first dissolving amphiphilic block copolymers in an organic solvent, which is a good solvent for all the block segments, followed by hydration of the solution. The hydration can be done by either slowly adding water to the organic 
polymer solution or by injecting the organic solution into water. This procedure renders the hydrophobic blocks insoluble, triggering copolymer self-assembly into polymersomes as a result of increasing interfacial tension between the hydrophobic blocks and water. Therefore, this technique is also called "solvent-switch" or "phase inversion" technique.

Amphiphilic block copolymers can self-assemble into various morphologies like vesicles, spherical and cylindrical micelles, determined by the interfacial area, chain length and volume of the hydrophobic part of the polymer [92]. In the classical description, for PEG based block copolymers possessing high interaction parameter with water, the vesicular structures are favored when the mass or volume fraction of the PEG $\left(f_{\mathrm{PEG}}\right)$ is between 10 and $40 \%$ (At $f_{\mathrm{PEG}}=45-55 \%$, cylindrical micelles tend to form, and at $f_{\mathrm{PEG}}=55-70 \%$, spherical micelles are predominantly formed) [78]. The polymer architecture can have significant influence on the PEG volume fraction for the formation of polymersomes. For example, Bae and coworkers prepared a series of $\mathrm{AB}_{2}$ type 3-miktoarm PEG- $b$-(PLLA) $)_{2}$ copolymers, which mimic the natural structure of phospolipids, and these copolymers showed much broader PEG volume fraction range (0.2-0.7) than their linear diblock counterparts (0.2-0.4) [93]. The vesicular formation can also be affected by the preparation pathway and conditions like polymer concentration, the type of organic solvent and the volume ratio of solvent to water. The size and size distribution of the polymersomes can also be manipulated by selecting different organic solvents or adjusting the stirring rate [94].

Another polymersomes formation method based on organic solvents involves the use of 
“water-in-oil-in-water" (W/O/W) double emulsions as templates. In this method, W/O/W double emulsions with a core-shell structure are first prepared in capillary microfluidic devices. Amphiphilic diblock copolymers were dissolved in a volatile organic solvent and used as the middle phase, where the subsequent solvent evaporation would lead to the polymersome formation [95]. Weitz and coworkers investigated the evolution of structure during solvent evaporation, and found that the initially homogeneous organic phase underwent a wetting transition, which they called it "dewetting". They proposed that it is important to consider the concentration of diblock copolymer in the middle phase of the double emulsion, and the presence of excess polymer could result in polymer shells with inhomogeneous thickness [96]. Later, they modified this method by adopting microfluidic devices for fabricating monodisperse polymersomes with biocompatible and biodegradable diblock copolymer poly(ethylene-glycol)-b-poly(lactic acid) for efficient encapsulation actives [97]. Furthermore, they fabricated non-spherical multi-compartment polymersomes from W/O/W double emulsions with different morphology as templates using glass capillary microfluidics [98].

There are some other ways to prepare polymersomes. Hauschild et al. reported a novel technique for the preparation of nano-sized, unilamellar polymersomes having a narrow size distribution by inject printers. In this technique, organic solutions of copolymers were injected drop-wise into water using the same technology used by inkjet printer, and the size could be controlled via the amphiphile concentration and cartridge type [99]. In addition, Maglio et al. reported a W/O emulsion-melting-sonication technique, which produced self-organized vesicles from PEO-PCL 
copolymer [100]. Zhang et al. also developed a unique polymersome system having asymmetric bilayer membrane formed by phase-guided assembly. By adding two diblock copolymers, poly(ethylene glycol)-block-poly(e-caprolactone) (PEG-PCL) and dextran-block-poly(e-caprolactone) (DEX-PCL), into a dextran/PEG aqueous two-phase system, DEX-PCL formed the inner leaflet around the dispersed dextran phase and PEG-PCL formed the outer leaflet with the PEG block facing the PEG continuous phase (Fig. 3) [88]. Finally, Holowka et al. prepared stable vesicles based on amphiphilic poly(L-lysine)- $b$-poly(L-leucine) block copolypeptides that formed stable vesicles in a aqueous solution, driven by the packing of the $\alpha$-helical poly(L-leucine) blocks [101].

Fig. 3

Despite the fact that the organic solvent based method is the most commonly used method, the residual organic solvent can induce biological toxicity. Furthermore, as traces of organic solvent can reduce the colloidal stability of polymersomes, considerably, extensive dialysis against water is often carried out to ensure the complete removal of organic solvent. However, this process would restrict the biomedical application of polymersomes using this method. In this perspective, the solvent free method has become increasingly more attractive.

\subsubsection{Solvent free method}

The solvent free method is based on the hydration of amphiphilic block copolymer films to induce self-assembly. In this method, polymers are first dissolved in an organic solvent and then a 
thin film is produced by evaporation of the organic solvent. Subsequently, the film is hydrated by the addition of water (this method is also called the "polymer rehydration" technique). The critical steps in the formation of polymersomes by the hydration procedure include: water permeation through defects in the polymer layers driven by hydration forces, inflation of polymer layers, and formation of bulges that finally yield vesicles upon separation from the surface [102]. In order to ensure the occurrence of mutual diffusion, an external energy source is required. The most common method used for overcoming the kinetic barrier is mechanical stirring. An alternative method is by using the electroformation of liposomes which is based on the hydration of the amphiphilic film under an a.c. electrical field that enhances water diffusion across the bulk copolymer [103]. Typically, the polymer rehydration technique produces polymersomes with a broad size distribution. Therefore, the final polymersomes are often size-regulated by sequential extrusion through filters with different pore dimensions under high pressure [104, 105].

As mentioned above, the size distribution of polymersomes produced by this method is polydisperse. Recently, Howse and coworkers have reported a method for the production of giant polymersomes having controlled size distributions by combining photolithography with bulk phase dewetting. This enables the spontaneous creation of unilamellar polymersomes with a narrow size distribution, regulated by the pre-patterned substrate [106]. An alternative direct rehydration process was also proposed by O’Neil and coworkers [107]. They demonstrated that polymersomes could be effectively formed by direct hydration of polymers composed of poly(ethylene 
glycol)-bl-poly(propylene sulfide) (PEG-PPS) AB diblock copolymers blended with poly(ethylene glycol) dimethyl ether (Mw 500 Da, or PEG 500 DME). This method leads to high encapsulation efficiency when compared to that typically observed by the thin film hydration method. Reversibly crosslinked temperature responsive nano-sized polymersomes was also prepared through the self-assembly of triblock copolymers poly(ethylene oxide)- $b$-poly(acrylic acid)- $b$-poly(N-isopropylacrylamide) (PEO-PAA-PNIPAM) followed by crosslinked at the interface using cysteamine [108]. The crosslinked polymersomes showed remarkable stability against high salt conditions and change of temperature in water, but they could be rapidly dissociated under reductive conditions mimicking the intracellular environment (Fig. 4). In summary, the direct hydration method is normally limited to polymers with relatively high chain flexibility, thus it cannot be used for glassy and crystalline copolymers because of the kinetic restriction.

Fig. 4

\subsection{Nanoparticles}

In this section, nanoparticle refers to homogeneous nanosized solid particle. Nanoparticles are widely used as drug carriers, in which drugs can be either entrapped inside or adsorbed on the surface. Compared to other drug carriers, nanoparticles are stable and tight, and can be easily prepared and piloted. Drug-loaded nanoparticles have been applied for subcutaneous or intravenous injection, oral administration and so on. 
For the preparation of nanoparticles, most methods are carried out in the emulsified system, involving two steps: preparation of an emulsified system, and the formation of nanoparticles by precipitation/gelation of a polymer or by polymerization of monomers [109]. A few other methods, which do not require the preparation of an emulsion during nanoparticle fabrication, are also demonstrated. For example, the microfabrication process has been used to make nano/microparticles with a monodisperse size distribution [110], where solid templates were used to make particles. Different than the solid template approach, Park and coworkers developed a hydrogel template approach and demonstrated that this simple process could prepare nano/microparticles at a fast rate, suitable for scale-up production [111]. In addition, nanoparticles can be fabricated through an inter/intramolecular crosslinking process $[112,113]$. However, the emulsification and emulsion polymerization pathways remain to be the most commonly used methods for the preparation of polymeric nanoparticles $[114,115]$.

\subsubsection{Emulsification}

The first step of the emulsification method is the preparation of an emulsified system. Typically, this step is accomplished by a high-energy emulsification technique such as mechanical shearing, creating emulsions with droplets of uniform size [116]. The droplet size and the distribution of emulsions can significantly affect the physical, physicochemical and organoleptic properties of the emulsified system. Narrowly-dispersed emulsion can be made by using the techniques such as 
capillary [117], microchannel emulsification [118], straight-through microchannel emulsification [119] and microfluidic approach [120]. The size-controlled narrowly-dispersed emulsion can be formulated by the membrane emulsification method [121-123].

Apart from the simple O/W emulsion system [124], novel emulsions having multiple components such as W/O/W [125, 126], water-in-oil-in-oil (W/O/O) [127] and solid-in-oil-in-oil (S/O/O) [128] were demonstrated, and they can lead to higher emulsion stability and drug loading efficiency. Furthermore, the applications of mini-emulsions, nano-emulsions and micro-emulsions instead of the classical emulsions were also demonstrated [129-132].

The second step of the emulsification method to fabricate polymeric nanoparticles usually involves a precipitation method. In this step, solvent of emulsions can be removed from the organic phase by various pathways such as solvent evaporation [133, 134], fast diffusion after dilution [135], or salting out [136] process. Polymeric nanoparticles can also be produced by the gelation of droplets in the emulsion $[137,138]$.

\subsubsection{Emulsion polymerization}

The emulsion polymerization method is another well-known technique to fabricate polymeric nanoparticles. However, in most cases, the polymerization process can lead to non-biodegradable nanoparticles, unsuitable for medical applications in the human body. So far, only a few monomers are suitable to produce biodegradable nanoparticles for in vivo applications, where the most notable 
system is alkylcyanoacrylates (ACA) and their derivatives $[139,140]$. The ACA monomers are very reactive, and the anionic polymerization process is usually spontaneously and can be quickly initiated by small amounts of a weak base such as hydroxyl ions of water (the traditional radical emulsion polymerization is not applicable to ACA). Thus, poly(alkylcyanoacrylate) (PACA) nanoparticles can be prepared by anionic emulsion polymerization of monomers initiated by not only the hydroxyl groups in water, but also any types of nucleophilic groups in the molecules dissolved in the polymerization system. To slow down the polymerization rate, the polymerization process is often performed in the acid conditions to enable the formation of nanoparticles instead of polymer aggregates [141]. In addition, suitable nucleophilic components that can initiate the polymerization of alkylcyanoacrylates are often embedded in the polymer structure to facilitate the nanoparticle formation. Other emulsion polymerization methods, such as redox radical emulsion polymerization and mini-emulsion polymerization, can also be used to prepare PACA nanoparticles [131, 142-146].

The main problem for the emulsion polymerization method is that some unreacted monomer or oligomer may be present in the formed nanoparticles, while this may cause toxic in clinical use.

\subsection{Nanocapsules formed on sacrificial templates}

Nanocapsules are hollow spherical structures with dimensions in the sub-micrometer region. Typical nanocapsules are composed of a polymer shell and a hollow inner space. Numerous approaches have been developed to fabricate nanocapsules. In order to distinguish them from 
polymersomes, we will focus on the nanocapsules that are formed on sacrificial templates in this sub-section. Generally, polymer shell is formed around a pre-formed template particle that can be subsequently removed, resulting in the formation of an empty polymeric shell [147].

\subsubsection{Layer-by-layer self-assembly}

A popular method to fabricate polymeric shell based on template particles is through the charged surfaces of polyelectrolyte self-assemblies [147-149]. In this approach, oppositely charged polyelectrolytes are used to apply layer-by-layer ( $\mathrm{LbL})$ deposition steps, resulting in the formation of multilayered polyelectrolyte shells. The thickness of the polymeric shell can be controlled by the number of polyelectrolyte layer [150]. Briefly, solid particles with charged surface are used as the templates, followed by immersing the template particles into the solution of polyelectrolytes carrying opposite charges. Consequently, a polymeric layer is formed on the surface through electrostatic interactions. As the surface charge of the polymer-coated particles converses, the free ionic groups can adsorb another layer of polyelectrolytes with opposite charges.

A class of commonly used templates for LbL electrostatic self-assembly is functionalized polystyrene latex particles bearing surface charges. For instance, sulfonated polystyrene beads were good templates, where chitosan and polyglutamic acid with low molecular weights could be alternately assembled onto the particles [151]. The template polystyrene core was subsequently removed by dissolving the nanoparticles in tetrahydrofuran. The sizes of the nanocapsules obtained 
were found to vary from 110 - $990 \mathrm{~nm}$, depending on the size of the polystyrene cores used.

Silica nanoparticles and mesoporous silica particles, which are slightly negatively charged, are another type of templates suitable for polyelectrolyte self-assembly. Itoh et al developed nanocapsules composed of chitosan (CS) and dextran sulfate (DexS) through the LbL approach [152]. Cationic CS was first adsorbed onto the surface of the template particles, and anionic DexS was then absorbed to form the next layer. After repeated LbL deposition of CS and DexS and etching of the silica cores using hydrofluoric acid (HF), biodegradable hollow capsules were obtained. Mesoporous silica particles can offer some unique advantages. For example, a model drug, fluorescein isothiocyanate (FITC) labeled albumin, could be first loaded into the pores of mesoporous silica particles [153], where the nanocapsules were formed by the LbL assembly of CS and DexS. As a result, after removal of the silica nanoparticles, drug-loaded hollow CS/DexS particles were formed.

Besides the nanocapsules composed of oppositely-charged polyelectrolytes, non-ionic polymeric shells can also be fabricated via stereo-complexation. Kida et al [154] reported a hollow sphere composed of poly(methyl methacrylamide) (PMMA). The PMMA stereo-complex, a double-stranded helical assembly formed between isotactic (it) and syndiotactic (st) PMMA units, was used as the component of porous membranes for artificial dialysis. These membranes contained hollow capsules made of PMMA stereocomplex multilayered shells using the alternating LbL assembly approach involving it- and st-PMMA based on the silica template. Van Der Waals interactions are believed to be responsible for the LbL deposition. 


\subsubsection{Surface initiated in-situ polymerization}

The surface-initiated controlled or "living" radical polymerization method is an effective approach for preparation of inorganic-organic core-shell hybrid nanoparticles having controllable shell structure and thickness. Accordingly, the nanocapsules were achieved by removing the core templates from the core-shell hybrid nanoparticles. In this method, silica nanoparticles are commonly used templates because they can be easily modified and etched. In particular, the hydroxyl groups on the silica surface template can be modified and used as initiators for polymerization of suitable monomers. In "living" polymerization processes, such as surface initiated atom transfer radical polymerization (SI-ATRP), block polymers can be synthesized by the addition of different monomers sequentially. Different from the LbL self-assembly method, the polymer shells that are formed via in-situ polymerization at the particle surface usually need to be stabilized by crosslinking before the removal of the core templates.

Some examples are as follows. Mu et al. [155] developed a pH-responsive nanocapsule using the SI-ATRP method. First, the bromoacetamide groups were conjugated onto the surface of the silica nanoparticles. The atom transfer radical polymerization (ATRP) of t-butyl acrylate and styrene was subsequently initiated to occur at the nanoparticle surface. The polystyrene outer layer was crosslinked by directly exposing the core-shell particles to UV radiation (365 nm). After etching the silica templates with $\mathrm{HF}$ and deprotection of the $t$-butyl alcohol side groups, the carboxyl-functionalized crosslinked polystyrene nanocapsules were obtained. When the 
co-monomers were changed into t-butyl acrylate, N-isopropylacrylamide (NIPAM) and $\mathrm{N}, \mathrm{N}^{\prime}$-methylenebisacylamide (MBA), crosslinked nanocapsules with dual $\mathrm{pH}-$ and temperature-sensitive shell were fabricated via a similar approach [156]. In addition, magnetic molecules such as $\mathrm{Fe}_{3} \mathrm{O}_{4}$ could be mixed into the silica cores [157]. After SI-ATRP and etching of the silica cores, nano-capsules containing magnetic particles were constructed.

In addition to ATRP, reversible addition-fragmentation chain transfer (RAFT) polymerization is another "living" polymerization process that can be used in this method. Huang et al. [158] reported the fabrication of nanocapsules having size between 450 and $900 \mathrm{~nm}$ using the RAFT polymerization approach. Amino silica nanoparticles were first functionalized by aminosilane agents, and then the dithiocarbonate chain transfer agent (CTA) was conjugated to the amino-functionalized surface of silica nanoparticles. Block copolymers were obtained at the particle surface by copolymerization of tert-butyl methacrylate (tBMA) and 2,3-dimethylmaleic imidopropyl methacrylate (DMIPM), followed by successive initiation of 2-hydroxypropyl methacrylamide. With UV crosslinking of the polymer shells and subsequent dissolution of the silica cores in an $\mathrm{NH}_{4} \mathrm{~F} / \mathrm{HF}$ buffer solution, hollow nanocapsules were obtained.

\subsubsection{Polymer monolayers formed by facial adsorption}

Besides the electrostatic interactions in the LbL assembly, other forces were also used to induce the adsorption of polymers on the surfaces of nano-sized sacrificial templates. Different from the 
multilayered hybrid nanocapsules formed via the LbL self-assembly method, the adsorbed polymer monolayers need to be crosslinked in order to gain stability. Some examples are as follows.

Gold nanoparticles (AuNPs) were found to be good sacrificial templates. To be specific. gold nanoparticles can enable the chemical interactions with certain functional groups, such as thiol or dithiocarbonate groups. In a study by Boyer et al [159], when oligoethylene glycol acrylate (OEG-A) and maleic anhydride block polymers were synthesized via RAFT polymerization, the chain transfer agent at the end of maleic chain could be adsorbed onto AuNPs. The polymer layer, which surrounded the nanoparticles, could be subsequently crosslinked using a small diamine molecule. The removal of the gold cores using aqua regia thus led to the formation of stable, biocompatible, and antifouling hollow polymer nanocapsules. Similarly, AuNPs can bind with thiol-containing polymers, which can also be assembled into polymer shells. For example, Sun et al. [160] demonstrated the fabrication of nanocapsules composed of polycyclodextrin. In this study, thiol- $\beta$-cyclodextrin ( $\beta$-CD-S) was first adsorbed onto AuNPs, where the aqueous suspension containing $\beta$-CD-S/Au nanoparticles was then added to the dilute solution of excess $\mathrm{I}_{2}$ in aqueous $\mathrm{KI}$ for further oxidation. During oxidation, the Au-S bonds were cleaved while the S-S bonds were formed simultaneously, resulting in the formation of hollow polycyclodextrin nanocapsules.

The $\mathrm{Au}(0)$ surface has also been shown to adsorb terminal alkyne groups, forming relatively densely packed and stable monolayer. Zhang et al. [161] demonstrated that AuNPs could be used not only as the template for formation of polymer shell, but also as the catalyst for cross-linking reaction. 
In their study, linear polymers carrying pendant propargyl ether groups were adsorbed onto the surfaces of AuNPs, where the crosslinking reaction occurred between the alkyne groups (Fig. 5). No additional crosslinking reagents or synthetic operations were required. Clearly, the gold(0)-catalyzed crosslinking reaction is a facile tool to synthesize nanopods.

Fig. 5

Ionic polymeric nanoparticles have also been used as sacrificial templates. For example, polyacrylic acid or polymethacrylic acid can form solid cores with polystyrene, and polycations can be adsorbed onto them accordingly. In the study by Liu et al. [162], they demonstrated the fabrication of chitosan nanocapsules by covering CS around the poly(styrene-co-methacrylic acid) (poly(S-co-MAA)) particles. After crosslinking of the polymer shells and etching of the cores, nanocapsules could be obtained. In addition, cationic functional molecules can be trapped by the negatively charged polymer monolayer. For example, Du et al. [163] synthesized $\mathrm{Fe}_{3} \mathrm{O}_{4}$ nanoparticles at the surface of the poly(styrene-co-acrylic acid) templates, followed by the coverage of CS monolayer. After crosslinking of the shells and removal of the cores, magnetic particles could be entrapped in the nanocapsule shells.

\subsubsection{Self-templating}

Recently, a "self-templating" method has also been developed to fabricate nanocapsules. In this 
method, both the template cores and the polymer shells are formed from the same polymeric materials. One example study was demonstrated by Liu et al. [164]. In this study, poly(vinyl acetate) (PVAc) lattices were prepared by emulsion polymerization. The surface segments of PVAc lattices were hydrolyzed into poly(vinyl alcohol) (PVA) segments. Subsequently, the surface PVA segments were crosslinked using glutaraldehyde (GA). The crosslinked polymeric nanocapsules were achieved after the PVAc molecules encapsulated within the crosslinked polymer shells were removed by dissolution in methanol. A similar method was reported by Dong et al. [165]. In their study, poly(tBA-co-NIPAm-co-AA) terpolymer nanoparticles were synthesize via soapless emulsion polymerization. The surface carboxyl groups of the terpolymer nanoparticles were then crosslinked with calcium ions. Subsequently, the cores were removed with acetone, rendering the formation of hollow nanocapsules.

Moreover, nanocapsules have been designed and prepared by partial removal of the polymeric self-assembled nanostructures or even unimolecular polymeric templates, rendering the shell-crosslinked hollow nanostructures. Wooley and co-workers developed an amphiphilic shell cross-linked knedel-like (SCK) nanostructures as the precursors for preparing nanocapsules, which are formed by self-assembly of the poly(isoprene- $b$-acylic acid) diblock copolymer in aqueous solution, followed by crosslinking of the poly(acylic acid) shell with diamino crosslinkers [166]. The nanocapsules were then prepared by selective removing the polyisoprene cores by oxidative scission with ozone, followed by reduction with sodium sulfite. The diameter of the nanocapsules was found 
to be affected by the length of the diamino crosslinker, which increased from $75 \pm 10 \mathrm{~nm}$ to $130 \pm 35$

$\mathrm{nm}$ based on TEM measurements by replacing diamino ethylene glycol with diamino poly(ethylene glycol) as the crosslinker. In a subsequent study of the same group, a similar strategy was applied to fabricate a nanocapsule based on a unimolecular brush copolymer [167]. Following the peripheral crosslinking of the poly(acylic acid) segments of the brush copolymer, the polyisoprene-based core of the brush copolymer was selectively degraded with ozone, resulting the formation of poly(acylic acid)-based nanocapsule. In addition, unimolecular dendritic macromolecules containing an inner porphyrin template were developed through a "unimolecular imprinting" approach [168]. The shell-crosslinked dendrimers were prepared by covalent attaching vinyl-containing dendrons to a porphyrin core, and then by crosslinking of the vinyl end-groups. After removing the porphyrin template core through hydrolysis, nanocapsules were obtained as potential synthetic host molecules with nearly homogeneous binding sites.

\subsection{Nanogels}

Polymeric nanogels are swellable nanosized hydrogel dispersions fabricated by physical or chemical crosslinking of hydrophilic or amphiphilic polymer chains [169, 170]. Various approaches have been reported for the preparation of polymeric nanogels, such as emulsion polymerization, precipitation polymerization, self-assembly, and "one-step" polymerization in a homogenous solution. 


\subsubsection{Emulsion polymerization}

The emulsion polymerization method has been widely used to fabricate nanogels. With this method, polymeric nanogels can be prepared by radical copolymerization of hydrophilic or water-soluble monomers in the presence of difunctional or multifunctional crosslinkers. For example, DeSimone and coworkers have synthesized nanogels by copolymerization of 2-acryloxyethyltrimethylammonium chloride (AETMAC) and 2-hydroxyethylacrylate (HEA) using poly(ethylene glycol) diacrylate (PEGDA) as a crosslinker for cellular gene and antisense delivery [171]. The size of the nanogels was found to be independent of the concentration of the crosslinker in heptane due to the poor solubility of these polymers. Nanogels containing charged monomer showed higher swelling ratios than the nonionic nanogels, and a higher crosslinking density could result in a lower swelling ratio. Although positive charge emerged in the nanogel surface, acceptable in vitro biocompatibility was found after the incubation with the HeLa cell line. The ionic nanogels were found to be resistant to aggregation and exhibited good stability in water, making these materials suitable for biomedical applications such as gene and antisense delivery. Smedt and coworkers have reported dextran-based cationic nanogels through copolymerization of dextran hydroxyethyl methacrylate and cationic methacrylate [172]. These nanogels could entrap short interfering RNA (siRNA) with a high loading efficiency due to electrostatic interactions. These nanogels are biodegradable due to the presence of ester bonds in the side chains of polymethacrylate derivatives, 
allowing for the sustained release of encapsulated siRNA and the efficient gene silencing over several days. As the degradation kinetics of nanogels can be easily tailored, these materials show great potential for the intracellular controlled release of siRNA.

Various functionalities can be introduced into nanogels through the incorporation of different monomers. For example, Nagasaki and coworkers have synthesized a series of nanogel, containing a crosslinked PEAMA core and a PEG shell, by using emulsion copolymerization [173-175]. Gold nanoparticles (GNPs) could be effectively incorporated into the core at low $\mathrm{pH}$ through the self-reduction of chloroaurate ions without the need for reducing agents. The GNPs incorporated nanogels exhibited photothermal efficiency, which could not only trigger the release of the payload, but also induce cell apoptosis due to the irradiation-induced thermal energy. Notably, after the modification with FITC-labeled DEVD peptides, nanogels exhibited less fluorescence in the normal cells due to the fluorescence resonance energy transfer process between GNPs and FITC, whereas marked fluorescence signals were observed in apoptotic cells because of the cleavage of DEVD peptide by caspase-3 and the release of FITC [176]. The fluorescence quenching nanoprobe can provide an effective means in monitoring the early response of cancer cells for therapeutic treatment as well as in high-throughput testing of anticancer drugs using the cell culture approach. In addition, Berkland and coworkers have reported an acid-sensitive nanogel system by polymerization of N-vinylformamide using 2-bis[2,2'-di(N-vinylformamido) ethoxy]propane as the crosslinker [177]. This nanogel system could be dissociated at low $\mathrm{pH}$ due to the cleavage of acid-labile ketal structure, 
leading to an acid-accelerated release profile of the payload. After hydrolysis of the aldehyde groups, these nanogels formed complexes with plasmid DNA in the presence of primary amines. The acid-labile nanogels having low charges showed more sustained gene transfection and low cytotoxicity compared to the highly charged nanogels.

NIPAM has been widely used as a monomer to prepare thermosensitive nanogels, of which the size could be finely tuned by controlling the temperature [178-180]. For example, thermosensitive and hydrolytically degradable nanogels were prepared by emulsion polymerization of NIPAM and dextran-lactate-2-hydroxyethyl methacrylate (DEXlactateHEMA) [181]. It was found that this nanogel system became smaller and more rigid with degradation. Liu and coworkers have reported thermosensitive nanogels based on P(NIPAM-co-NPTUA) for selective detection of $\mathrm{Hg}^{2+}$ through emulsion polymerization [182]. The NPTUA-labeled nanogels could react with $\mathrm{Hg}^{2+}$, resulting in a prominent blue shift of the fluorescence emission peak. The nanogels showed high selectivity and sensitivity for $\mathrm{Hg}^{2+}$ with a detection limit at the nanomolar level at room temperature, which was enhanced due to the collapse of the nanogels at elevated temperatures. Monomethyl oligo(ethylene glycol) acrylate has also been a popular monomer for the preparation of thermosentive nanogels. Li and coworkers prepared thermosensitive nanogels by copolymerization of monomers containing oligo(ethylene glycol), ortho ester and disufide linkage [183]. At temperatures above the phase transition temperature, the nanogels exhibited a hydrophobic nature, resulting in an enhanced drug loading capacity. It was shown that the drug-loaded nanogels had a good stability in the normal 
condition while the drug release could be triggered under bio-related stimuli, due to the existence of acid-labile ortho ester and reduction-cleavable disulfide linkage (Fig. 6).

Fig. 6.

The controlled/living radical polymerization techniques, such as ATRP, have also been used in mini-emulsion for the preparation of nanogels with well-controlled polymer segments. For example, Matyjaszewski and coworkers have prepared well-defined nanogels using the inverse mini-emulsion ATRP method [184-186]. The two-step sequential emulsion copolymerization approach resulted in the formation of a new type of hairy nanogels through one-pot synthesis [187]. In their studies, uniform nanogels were prepared by the micro-emulsion ATRP method using methyl methacrylate (MMA) and ethylene glycol dimethacrylate (EGDMA) as the monomer and cross-linker, respectively. The micro-emulsion system were converted into an emulsion polymerization by addition of a second monomer, where linear arms grew from the remained initiating sites in the nanogels, rendering the formation of hairy nanoparticles in situ. Based on this approach, this research group used reduction-sensitive cross-linkers to construct nanogels for intracellular drug delivery, where various water-soluble biomolecules including anticancer drugs, carbohydrates and proteins were successfully loaded in the nanogels $[188,189]$. They demonstrated that the colloidal stability, swelling ratios, controlled degradability of the nanogels prepared by the ATRP method are superior to their counterparts prepared by the conventional free radical polymerization approach in inversed 
mini-emulsion.

\subsubsection{Precipitation polymerization}

Precipitation polymerization is another commonly used approach for preparation of nanogels, especially for thermosensitive nanogels (e.g., NIPAM based nanogels). When the polymerization temperature is above the lower critical solution temperature (LCST) of the resulting NIPAM-based polymers, the growing NIPAM chains would collapse when they reach a critical length. This process can lead to the formation of precursor particles [190]. Nanogels can be subsequently formed from precursor particles through three different mechanisms, including the continuous growth of the precursor particles by addition of monomers or macroradicals, deposition of the polymer chains on the surface of existing polymer particles, and aggregation of the precursor particles to form a large colloidally stable polymer particle. During precipitation polymerization, the charges originated from the initiator fragments as well as considerable water may be incorporated into the collapsed polymer chains. This differs from classical emulsion polymerization of water-insoluble monomers, in which the particles containing a compact structure are formed. After the polymerization process is completed and the temperature is reduced below the LCST, the formed nanogels can swell and incorporate abundant water.

Since the first example demonstrated by Pelton et al [191], the preparation of PNIPAM-based nanogels by the precipitation polymerization method has been extensively investigated [192-194]. Li 
et al. have prepared a series of core-shell nanogels with a thermo-responsive core and a pH-responsive shell via this method [195]. Recently, multifunctional nanogels have received increasing attention for their potentials in biomedical and pharmaceutical applications [196-198]. Yang and coworkers have synthesized a new class of temperature-sensitive nanogels based on poly( $N$-isopropylacrylamide-co-butyl methylacrylate) for this purpose [180]. These nanogels have potential applications as a blood-vessel-embolic material in the interventional therapy of liver tumors. In addition to the PNIPAM system, other temperature-responsive polymers were also demonstrated. For example, $\mathrm{Wu}$ et al. developed $\mathrm{pH}$ and temperature dual responsive nanogels, composed of hydroxypropylcellulose and poly (acrylic acid) with CdSe quantum dots (QDs) in the interior through an in-situ immobilization method [199]. The hybrid nanogels displayed good potential as a stimuli-responsive platform for sensing physicochemical environment, cell imaging and $\mathrm{pH}$-triggered anti-cancer drug delivery. Nie and coworkers also demonstrated a multifunctional nanogel for anti-tumor drug delivery application by the free radical precipitation polymerization approach [200]. They found that surface modification of the nanogels with iRGD could lead to enhanced intracellular uptake of nanoparticles by both vein endothelial cells (HUVECs) and extravascular tumor cells (B16). Moreover, the combined diagnostic and therapeutic features could be achieved by incorporation of bovine serum albumin - gold nanoclusters to the nanogels.

\subsubsection{Self-assembly}


The self-assembly method has also been extensively used to prepare nanogels. In this method, controlled aggregation of hydrophilic or amphiphilic polymers is formed by physicochemical interactions, such as hydrophobic interactions, electrostatic interactions, hydrogen bonding, stereocomplexation or supramolecular chemistry. The preparation of nanogels via self-assembly is usually carried out in aqueous media under mild conditions. Akiyoshi et al. demonstrated nano-sized hydrogels by the hydrophobic association of cholesterol-modified pullulan in the presence of insulin [201]. The nanogels were formed in a narrow range of cholesterol/sugar units ratio (1:40-1:100) with a diameter of 20-30 nm and they contained up to five insulin molecules per particle. Bronich and coworkers also demonstrated functional polymeric nanogels with an ionic core for delivery of chemotherapeutic agents [202]. Reductively labile disulfide bonds were incorporated in the ionic cores by using cystamine as a biodegradable cross-linker through electrostatic interactions.

Besides the formation of physical interactions, covalent cross-linking of amphiphilic polymers chains were also used in the preparation of nanogels, especially for the nanogels with a core-shell structure [170]. The cross-linkers display marked influence on the swelling behavior, pore size and morphology of the nanogels, and therefore the release kinetics of the entrapped drug molecules. To achieve the controlled delivery feature, various stimuli-responsive moieties, cleavable under $\mathrm{pH}$ change, redox environment, light, enzymes and etc., were introduced into the polymer networks. For example, Zhao and coworkers prepared thermal- and photo-responsive core-shell nanogels by temperature-sensitive self-assembly of a double-hydrophilic block copolymer in aqueous solution, 
followed by reversible photo-dimerization and photo-cleavage of coumarin moieties [203]. Chen and coworkers also prepared $\mathrm{pH}$-responsive biodegradable polypeptide nanogels as potential drug carrier by photo-crosslinking [169]. The drug-loaded nanogels exhibited the release profiles dependent on the external $\mathrm{pH}$ value as a result of the $\mathrm{pH}$-responsive units in the polymer. Due to the marked difference in the redox potential between the extracellular and intracellular spaces, the nanogels crosslinked by reduction-responsive linkages, especially the disulfide bonds, that can be selectively decrosslinked in the intracellular space, have received a great deal interest for intracellular drug delivery. Zhong and coworkers developed the reduction-responsive dextran nanoparticles by the self-assembly of lipoic acid-modified dextran in aqueous media, followed by the formation of intermolecular disulfide linkages between the lipoic acid units and dithiothreitol (DTT) [204]. The reduction-triggered release of the doxorubicin-loaded nanoparticles was observed in an in vitro study. Thayumanavan and coworkers also synthesized a polymethacrylate derivative containing oligo(ethylene glycol) (OEG) and pyridyldisulfide [205]. The disulfide-crosslinked nanogels could be prepared by the formation of nanoaggregates in water via self-assembly, followed by the formation of intermolecular disulfide linkages via thiol-disulfide exchange reaction.

\subsection{4 "One-step" polymerization in homogenous solution}

Fabrication of nanogels via "one-step" polymerization (ROP) in homogenous media has attracted

a great deal of interest recently [206]. This approach often involves the use of difunctional or 
multi-functional monomers. For instance, the microscopic polyacrylate networks were synthesized by atom transfer radical copolymerization of acrylate derivatives and diacrylate comonomers [207, 208]. To avoid the formation of macroscopic gelation, highly diluted monomer solutions were used. Nanogels could also be prepared via "one-step" ring-opening polymerization (ROP) in homogenous media, where difunctional ROP monomers are initiated by using a macro-initiator with or without other comonomers [209]. For example, Wang and coworkers synthesized various difunctional phosphate monomers, and nanogels having PEG as the shell and polyphosphoester as the crosslinked core was obtained by ROP of the difunctional phosphate monomers [210, 211]. Reduction-responsiveness could be incorporated in the nanogels by the introduction of disulfide linkages to the difunctional phosphate monomers [211]. The release of vancomycin from the nanogels was markedly accelerated by the addition of alkaline phosphatase or DTT. In two separate studies, a difunctional L-cystine $N$-carboxyanhydride (L-Cys NCA) containing a disulfide bond was synthesized [212, 213]. Disulfide core-crosslinked nanogels with the PEG shell and the crosslinked polypeptide core were prepared by ROP of L-Cys NCA and comonomers using amino-terminated PEG as a macro-initiator. The enhanced drug release rates were observed in the presence of intracellular reducing agent, glutathione $(\mathrm{GSH})$. The pH-responsiveness could also be introduced into the nanogels by copolymerization of amino acids containing ionizable side groups [214]. In this case, ionizable anti-cancer drug, e.g., DOX, could be loaded into the nanogels having oppositely charged core through electrostatic interactions, resulting in nanogels with high drug loading capacity 
(DLC) and drug loading efficiency (DLE).

\subsection{Nanofibers}

Nanofibers are generally defined as fibers with diameters equal to or less then $100 \mathrm{~nm}$. This definition is sometimes extended to include fibers with diameters measured less than $1000 \mathrm{~nm}$ [215]. Conventional fiber-spinning technologies cannot produce robust fibers with diameter smaller than 2 $\mu \mathrm{m}$ because of the physical limitations in the process. In contrast, polymeric nanofibers for biomedical applications can be fabricated by a range of techniques including electrospinning, phase separation, membrane templating, self-assembly, and etc. [215, 216]

\subsubsection{Electrospinning}

Electrospinning is the most versatile method for the fabrication of polymeric nanofiber. Electrospun polymeric nanofibers are formed by stretching a jet using electrostatic forces from a liquid polymer solution or melt [217-221]. The parameters affecting the electrospinning process include molecular weight, molecular-weight distribution, polymer architecture, surface tension, viscosity, solvent, solution conductivity, voltage, feed rate, temperature, diameter of the spinneret, distance between the tip and collector, humidity, and etc. By varying the above parameters, polymeric nanofibers with diameters ranging from a few nanometers to several micrometers can be obtained from a variety of polymers [222-225]. Despite the great potential of this technique, the 
conventional electrospinning process of polymer solution suffers from a number of drawbacks including the low production rate (up to $300 \mathrm{mg}$ per hour per spinneret); need of solvent extraction; lack of suitable solutions for certain polymers at the ambient temperature; and environmental concerns when hazardous solvents are used [216]. In comparison, the melt electrospinning process is free from the use of solvent and has undeniable advantage in terms of environment friendliness [226-228]. Nanofibers of biodegradable polymers including poly(lactic acid) (PLA), poly( $\varepsilon$-caprolactone) (PCL), and PEG-PCL have been fabricated using the melt electrospinning technique [229-231]. Generally speaking, much less studies have been carried out by the melt electrospinning technique than the solution electrospinning technique. This is because of the former requires much higher viscosity from the molten polymer, where the degradation of certain polymers of interest at high temperatures can be an issue [216]. To increase the productivity of solution electrospinning, several approaches including the increase in the number of spinnerets were used. Multiple-jet electrospinning and needleless electrospinning have found to be able to provide high production rate of polymeric nanofibers [217, 232-237]. For the creation of polymeric nanofiber with unique micro-structure, such as core-sheath nanofibers, modification of the spinneret and/or the type of solution was also applied (e.g., coaxial and emulsion electrospinning) [238-243].

\subsubsection{Phase separation}

The phase separation method can also be used to fabricate a nanofibrous matrix. This method 
would involve a series of preparation steps: dissolution of polymer, gelation of polymer, extraction of solvent with water, and freeze-drying of the polymer scaffold [244-246]. The parameters affecting the morphology of the final nanofibers include polymer concentration, crystallization ability of polymer, annealing temperature, gelation temperature, and freezing temperature. For example, Ma et al. reported the creation of electroactive biodegradable nanofibrous biomimetic scaffolds from the blends of polylactide and electroactive degradable tetraaniline-polylactide-tetraaniline (TPT) copolymer via the thermally induced phase separation technique. The diameters of the resulting fibers could be controlled between 200 and $500 \mathrm{~nm}$ through the adjustment of polymer concentration, phase separation temperature and TPT content in the blends [247]. Although the phase separation technique requires simple equipment in lab scale, it is difficult to scale up due to the requirement of multiple and relatively complex steps as well as the limitation of specific polymer that can be used [248].

\subsubsection{Membrane templating}

The membrane templating method can be used to create nanofibers within the cavity (i.e., nanochannels) of nanoporous membranes (with 5-50 $\mathrm{mm}$ thickness) [244]. Because the nanochannels in the membranes are very uniform in size, the diameter and the aspect ratio of the nanofibers fabricated by the membrane template approach can be precisely controlled [249]. With this method, $\mathrm{Xu}$ et al. reported the fabrication of nanofibers from biocompatible and biodegradable 
polymer PCL using an aluminum oxide membrane as the template. The nanostructure morphology can be controlled by the processing parameters and template design $[250,251]$. The membrane templating method has several advantages. For one, a wide range of polymers can be used to fabricate nanofibers. Second, polymeric nanofibers with different diameters and lengths can be prepared using different templates. However, this technique is also difficult to scale up and thus with limited commercial value [216].

\subsubsection{Self-assembly}

Self-assembly is an autonomous process, in which the disordered system of pre-existing components can organize and arrange themselves into ordered patterns or structures through non-covalent forces, such as hydrogen bonding, hydrophobic forces, and electrostatic interactions [248, 252], without human intervention. Peptide amphiphiles are most commonly used by the self-assembly method to produce nanofibers [253, 254]. This is because peptide amphiphile is a triblock polymer having three segments: a hydrophobic tail that can provide the hydrophobic driving force for self-assembly thus forming the core of the nanofiber, a region of beta-sheet forming amino acids than can stabilize the self-assembled nanofiber structure, and a peptide epitope that can enhance bioactivity and solubility of the molecule in water. As a result, nanofibers having diameters around 5-25 $\mathrm{nm}$ can be formed by the self-assembly process [248]. Based on this method, Tong et al. [255] reported a collagen-mimetic peptide amphiphile (CPA) system that could be self-assemble into 
nanofibers with structure and biological functions similar to native collagen fibers (Fig. 7). The parameters that can affect the morphology of peptide amphiphile nanofibers include the sequence and length of the peptide molecules, the geometrical constraints of the peptide molecules, and the interplay between the hydrophilicity and hydrophobicity [254]. Besides the peptide amphiphiles, other collagen-mimetic peptides, diblock copolymers, triblock copolymers, dendrimers, chitin, and bolaform of glucosamide and its deacetylated derivative can also be used to fabricate nanofibers through the self-assembly method [244, 256-258]. Self-assembly has some unique advantages such as controlled physical and biochemical properties of nanofibers through the control of composition and chemistry of amphiphilic peptides, easy incorporation of cells during the fiber formation, 3-dimensional pore arrangement, and injectable for in vivo assembly. However, it is a laboratory scale process that has limitations including the complex process, limited polymer structures, and limited fiber diameter $(\sim 2-30 \mathrm{~nm})$ and length $(\sim 10 \mu \mathrm{m})[244,248]$.

Fig. 7

\subsection{Others}

Dendrimers [259-261], brush polymers [262] and polymer nanocomposites [23] have also been considered as promising nanostructured biomaterials. Two distinct synthetic routes have been demonstrated for the preparation of dendrimers: the divergent and the convergent methods. In the divergent method, the molecular growth is initiated from the core and proceeds radially outward 
toward the dendrimer periphery. In the convergent method, the molecular growth starts at what will become the periphery of the dendrimer and thus proceeds inward [259, 263]. Both methods are stepwise, tedious and time-consuming processes as they include several protection-deprotection and extensive purification steps. The divergent method is more suitable for the syntheses at a larger scale and the preparation of higher generation dendrimers. Nevertheless, incomplete growth steps and side reactions could lead to the production of slightly imperfect samples. The convergent syntheses usually require only two simultaneous reactions for any generation-adding step, therefore making the purification of dendrimers relatively simple. However, the number of steps required to fabricate a large structure by the convergent method is the same as that for the divergent method, but more starting material is required for the former $[259,264]$.

Brush polymers, composed of densely grafted side chains along a polymeric backbone, can form spherical, cylindrical, or worm-like structures by varying the composition and chain length of either backbone or side chains [265]. Three main strategies have been established for the prepration of brush polymers: "grafting through" (the end-group polymerization of macromonomers) [265, 266], "grafting onto" (the construction of functionalized backbone and side chains separately followed by coupling reactions) [267], and "grafting from" (the polymerization of monomers from presynthesized backbones with multi-initiating sites) [268]. Within each strategy, various polymerization techniques such as anionic polymerization [269], ring-opening polymerization [270, 271], ring-opening metathesis polymerization [272-275], controlled radical polymerizations [271, 276, 277], and various 
coupling reactions (“click chemistry") [270, 272, 278] have been employed. An extensive review on the preparation of brush polymers can be found elsewhere [262, 279].

Polymer nanocomposites are another class of nanostructured materials, consisting of inorganic/organic fillers with nanometer scale dimensions (i.e., at least one dimension is within the range of 1-100 nm) and a polymer matrix. For biomedical applications, the demonstrated nanofillers include, but are not limited to, clays, hydroxyapatite, carbon nanotubes, silver, gold, titanium oxide, silica, nanocalcium carbonate, and nanocellulose crystals, whereas the biopolymer matrices include polysaccharides, aliphatic polyesters, polypeptides, proteins, and polynucleic acids [280-282]. These polymer nanocomposites can be prepared by techniques such as in situ polymerization, solution casting, melt processing, electrospinning, or supercritical fluid methods. The preparation schemes of polymer nanocomposites have been extensively reviewed elsewhere [282-284].

\section{Biomedical applications of polymeric nanostructured materials}

\subsection{Drug delivery}

In the past decade, various polymeric nanostructured materials have been used for the controlled delivery of a diverse range of bioactive molecules, including low molecular weight (LMW) drugs and peptides/proteins. The delivery of drugs by polymeric nanovehicles has exhibited significant advantages over the delivery of parent free drugs. These advantages include the improved water solubility, increased bioavailability, minimized deactivation potential, reduced antigenic activity, 
decreased systemic toxicity, and enhanced acceleration ability in the lesion site.

\subsubsection{Polymer-drug conjugates}

In 1975, Ringsdorf first proposed the concept of "polymeric prodrug", which provided the theoretical bases for the development of covalently bound polymer-drug conjugates [285]. In Ringsdorf's model, three components can be conjugated with the biocompatible polymeric backbone, i.e., (1) the hydrophilic segment, to ensure the solubility of the system in an aqueous environment; (2) the bioactive molecule, to play a role in the pharmacodynamics; and (3) the targeting agent, to specifically recognize the desired physiological destination. Based on the rational design of the pharmacologically active system, various polymer-drug conjugates have been exploited. In recent years, some of polymeric prodrugs have entered the clinical trial stage (Table 2) [286].

\section{Table 2.}

Many natural and synthetic polymers having different topologies, compositions and functional groups were used to construct polymer-drug conjugates, which could be spontaneously self-assembled into micelles or micelle-like nanoparticles (Fig. 8). These polymers include natural polysaccharides (e.g., hyaluronic acid, dextran, chitosan and heparin), liner hydrophilic synthetic polymers (e.g., $\mathrm{N}$-(2-hydroxypropyl) methacrylamide (HPMA) copolymers, poly(ethylene glycol) (PEG), poly(L-glutamic acid) (PGA), poly(L-aspartic acid) (PAA)), dendrimers, and some 
amphiphilic block copolymers (e.g., poly(ethylene glycol)- $b$-polylactide (PEG- $b$-PLA), poly(ethylene glycol)- $b$-poly(lactide-co-glycolide) (PEG- $b$-PLGA) and poly(ethylene glycol)- $b$-poly( $\varepsilon$-caprolactone) (PEG- $b$-PCL) copolymers). Some specific examples are as follows.

Fig. 8

\subsubsection{Polysaccharides}

Polysaccharides are an important class of natural polymers which have been widely used for the development of polymeric prodrugs. The unique properties of these polymers include the excellent biodegradability, biocompatibility, non-toxicity, non-immunogenicity and even some biological activities (e.g., targetability, and antiviral and antitumor activities) [310]. The pharmacokinetics of the corresponding prodrugs are greatly influenced by their electric charges, molecular weight, polydispersity, chemical modification and topology [311]. The chemical structures of polysaccharides typically used for conjugating antitumor drugs are illustrated in Fig. 8A.

In these polymers, hyaluronic acid is a linear anionic polysaccharide composed of alternating D-glucuronic acid and $N$-acetyl-D-glucosamine linked with the $\beta(1 \rightarrow 4)$ interglycosidic linkage. As a main component in the extracellular matrix, hyaluronic acid plays a critical role in the cellular growth, proliferation, differentiation, migration and even malignant metastasis [312, 313]. The elevation of hyaluronic acid is considered to be a reliable disease progression index in some types of malignant tumors (e.g., bladder cancer) [310]. Due to its strong affinity with cell-specific markers 
(e.g., cluster determinant 44 (CD44) $[314,315])$ and receptor for hyaluronate-mediated motility (RHAMM, overexpressed in many malignant cells and stem cells [316, 317]) that is directly correlated with the invasive properties and metastatic processes of aforementioned cells, hyaluronic acid has often been used for targeted drug delivery without any additional ligands. The hydroxyl and carboxylic groups in the hyaluronate backbone provide the appropriate sites for conjugating antitumor drugs, such as doxorubicin (DOX), paclitaxel (PTX), camptothecin (CPT), sodium butyrate and curcumin [310].

Dextran is another type of polysaccharide synthesized by certain lactic acid bacteria or dextransucrase, which consists of the $\beta(1 \rightarrow 6)$ linked D-glucose main chain with various ratios of linkages and branches. More than three dextran derivatives, including carboxymethyl dextran, oxidized dextran and amino dextran, have been used to conjugate different antitumor drugs, such as DOX, CPT, mitomycin C (MMC) and methotrexate (MTX) [318-321].

Chitosan is a linear polysaccharide containing $\beta(1 \rightarrow 4)$ linked 2-amino-2-deoxy-D-glucose and 2-acetamido-2-deoxy-D-glucose, which is the $N$-deacetylated derivative of chitin. Like hyaluronic acid, chitosan plays a remarkable role in malignant biology, which have been shown to inhibit tumor angiogenesis [322]. In addition to the chitosan molecules, several derivatives including glycol chitosan, $N$-succinyl chitosan and carboxymethyl chitosan have also been used to chemically conjugate with DOX, PTX and MMC.

Heparin is one kind of mucopolysaccharide polysulfuric esters having alternating units of 
sulfated glucuronic acid and structurally diverse glucosamine derivative jointed by the $\alpha(1 \rightarrow 4)$

linkage. It has been shown that the heparin molecules, especially LMW heparin, can inhibit malignant cell adhesion, deactivate heparanase, activate the attack by natural killer (NK) cells in the immune system, and interfere with the activities of some growth factors, such as basic fibroblast growth factor (bFGF) and vascular endothelial growth factor (VEGF) [44]. Through the above approaches, the presence of heparin can prevent tumor angiogenesis, exacerbation and metastasis. Similar to the abovementioned polysaccharides, the heparin molecules are also capable of conjugating with various biological molecules (e.g., PTX).

\subsubsection{Linear hydrophilic synthetic polymers}

Many polymeric prodrugs have been synthesized utilizing various linear hydrophilic synthetic polymers. As shown in Fig. 8B, HPMA copolymers, PEG, PGA, PAA, and their copolymers (PEG- $b$-PGA and PEG- $b$-PAA) are used to prepare the drug conjugates.

HPMA copolymers, which were first exploited by Duncan and coworkers, are widely used as hydrophilic and biocompatible matrices for drug carriers [323]. Diverse modifications of HPMA copolymers have been carried out by copolymerization with various functional monomers, where different antitumor drugs were combined with HPMA copolymers through facile synthesis techniques. In this system, the HPMA copolymer-DOX conjugate (PK1) was the first polymeric prodrug, which entered clinical trials in 1994 [324]. PK1 represents the first milestone for the 
development of polymer-drug conjugates. PEG (also known as poly(ethylene oxide) or (PEO)) having different molecular weight, low polydispersity and activation form, is another kind of widely used and versatile linear hydrophilic synthetic polymer for biomedical applications. PEG has been approved by the US Food and Drug Administration (FDA) as an excipient in various pharmaceutical formulations due to its negligible immunogenicity, antigenicity and toxicity. For example, PEG has been routinely used to modify different bioactive molecules (e.g. DOX) [325].

Synthetic poly(amino acid)s represents another kind of biocompatible and biodegradable polymers with many important applications. These polymers exhibit precise secondary conformations, and have been widely utilized in different biomedical realms, including drug and gene delivery, as well as tissue engineering [169, 326-329]. Among these polymers, PGA and PAA are the most promising candidates for drug conjugating ascribed to the respective pendent carboxyl groups of each L-glutamic acid and L-aspartic acid.

\subsubsection{Dendrimers}

Dendrimers (or dendritic polymers) are three-dimensional, highly ordered star-like oligomeric and polymeric macromolecules (Fig. 8C). The physiochemical properties, such as solubility, stability, functionality and charge density, are determined by the chemical structure of the monomer unit(s) in the core and and surface. In 1985, Tomalia and coworkers first deminstrated the preparation of polyamidoamine (PAMAM) dendrimers with precisely defined structures in a stepwise fashion [330, 
331]. Since then, many chemists have reported the different synthetic routes to prepare a wide range of dendrimers.

Compared with most of the synthetic polymers, the major advantage of using dendrimers for biomedical applications is their monodispersed chemical structures usually having a polydispersity less than 1.05. Due to the abundant and dense functional groups on the surface of the dendrimer, a large number of chemotherapeutics could be linked to the dendrimers, thus maximizing their ability for biological interactions [286]. However, the poor biocompatibility and biodegradibility of the dendrimers, and the relative difficult and costly syntheses are also noted in this system. These challenges need to be overcome before the successful implementation of clinical translation.

\subsubsection{Amphiphilic block copolymers}

The amphiphilic block copolymers are another kind of polymer system that can effectively incorporate and deliver anti-tumor drugs [312, 332-334]. In this system, PEG is the most commonly used hydrophilic block because of its minimal immunogenicity, high water-solubility, high hydration and flexibility, where biocompatible and biodegradable aliphatic polyesters and their copolymers, such as PLA, PLGA and PCL, are often chosen as the hydrophobic blocks (Fig. 8D). The selection of the hydrophobic moieties can notably change the properties of prodrug micelle, such as size, stability and drug release profile.

The system with hydrophobic linear aliphatic polyesters (e.g., PLA, PLGA and PCL) as the 
core-forming blocks has been approved by FDA for therapeutic applications. Generally, PLA can be synthesized by condensation polymerization of lactic acid or by ROP of lactide (LA). PLA has three different stereoisomeric forms: poly(L-lactide) (PLLA), poly(D-lactide) (PDLA) and poly(D,L-lactide) (PDLLA), due to the chiral nature of lactic acid. PLGA is a random copolymer of lactic acid and glycolic acid linked by ester linkage, which can be synthesized by ROP of LA and GA. PLGA exists in several distinct forms that can be controlled through the ratio of LA and GA. PCL is also a biodegradable polyester that can be prepared by ROP of CL. In an aqueous media, the amphiphilic block copolymers containing PEG and aliphatic polyesters can self-assemble into micelles or micelle-like aggregations, which is ideal to serve as drug carriers. A great amount of drugs, especially antitumor drugs (e.g. PTX, DTX, DOX and CPT), have been conjugated to the hydrophobic aliphatic polyester blocks [335-339], which moieties can adjust the characteristics of prodrug nanoparticles, such as the size and stability for clinical applications.

\subsubsection{Others}

In addition to the above-described systems, various drugs, such as PTX, DOX, and CPT were conjugated to hydrophobic PLA through the ROP of LA initiated by the hydroxyl group on the drug molecule [340-342]. The PLA-drug conjugates could form uniform nanoparticle through nanoprecipation, which would be further encapsulated by amphiphilic copolymers, such as PEG- $b$-PLGA. The obtained prodrug nanoparticles exhibited sustained drug release in the long-term 
detection (i.e., 14 days). In addition, the amphiphilic brush polymer-drug conjugates have been synthesized by ring-opening metathesis copolymerization and postpolymerization azide-alkyne click reaction [270, 273]. The developed prodrugs also exhibited controlled drug release, and effective endocytosis and cytotoxicity. Furthermore, there are other types of polymer-drug conjugates attracted the wide attention of researchers, such as peptide-drug and antibody-drug conjugates, and so on $[343,344]$. However, this is not the focus of this review.

\subsubsection{Encapsulation of low molecular weight drugs}

In addition to the chemical conjugation, physical entrapment is another vital approach to incorporate drugs into nanocarriers that can reduce the side effects and enhance the efficacy. We will briefly review the use of this approach to fabricate nanovehicles, including micelles, polymersomes, nanogels, to deliver bioactive LMW drugs.

\subsubsection{Polymeric micelles}

Polymeric micelles are nanoscale colloids that can be formed by self-assembly of amphiphilic copolymers in an aqueous environment, where the formed micelles consist of a hydrophilic shell and a hydrophobic core. Various hydrophilic flexible polymers have been used as the shell-forming moieties. For intravenous drug delivery applications, the hydrophilic blocks of the amphiphilic copolymers are often composed of zwitterionic materials or PEG, which can resist the nonspecific 
protein adsorption (i.e. non-fouling property) and prolong the circulation time of nanoparticles in the complex in vivo environment $[214,345-347]$. The most commonly chosen hydrophobic blocks include poly(propylene oxide) (PPO), biodegradable aliphatic polyesters (PLA and PLGA), and poly(amino acid)s (e.g. PGA and PAA), which can segregate and form micellar cores in the aqueous environment, where the segregation process is driven by a combination of physical interactions, including hydrophobic and electrostatic interactions, metal complexation, and hydrogen bonding [66, $326,328,346,348-355]$.

The hydrophobic core can serve as the sustained release reservoir of bioactive LMW drugs (e.g., antitumor drugs), whereas the hydrophilic shell can stabilize the hydrophobic core and make the micelle a stable vehicle for i.v. administration. The typical polymeric micelles have an average diameter between $10-200 \mathrm{~nm}$ but a narrow size distribution. These micellar sizes are similar with those of natural mesoscale vehicles (i.e., viruses and lipoproteins), thus possessing enhanced permeation retention (EPR) capability [90, 356].

Based on the promising results from clinical trials, polymeric micelles appear to be the most advanced platform for drug delivery. Table 3 lists six formulations to fabricate polymeric micelles that can encapsulate various LMW anti-tumor drugs such as PTX, DTX, DOX, oxaliplatin and cisplatin. The encapsulation process involves the physical entrapment or coordinate bonding of drug molecules with the hydrophobic segment in the micellar core. These formulations are in different stages of clinical trials for the treatments of various malignancies, especially for some advanced or 
metastatic cancers $[31,349,356]$. It is encouraged to learn that Genexol®-PM, composed of PEG-b-PDLLA and PTX, has been approved for the treatment of non-small cell lung cancer (NSCLC), and ovarian, breast and gastric cancers in Korea [356-358]. In addition, BIND-014, the first prostate-specific membrane antigen targeted PEG- $b$-PLA and PEG- $b$-PLGA mixed micelles, containing DTX, has also been approved for the phase II clinical trials in the treatment of advanced or metastatic solid cancers [4]. These polymeric micelle formulations exhibited some notable advantages, including the reduced side effects, rational drug metabolism, and improved efficacy and tolerance in the course of current clinical trials.

Table 3.

However, despite the significant progress made in the past decades, the therapeutic efficacy of the varying polymeric micelle prescriptions is still far from our expectation. In specific, the desired performance for this system is the programmable "on-demand" drug delivery capability, which is yet to be realized completely $[364,365]$. To achieve this goal, tremendous efforts have recently been made to develop "intelligent" micelles with the response ability to intracellular stimuli, i.e., $\mathrm{pH}$, redox potentials and enzyme, for the purpose of efficient intracellular drug delivery [366, 367]. One of the most promising means involves the incorporating stimuli-responsive cleavable linkages in the polymer structures. Once triggered by the intracellular microenvironment, the chemical structures of such macromolecules can be changed leading to the disintegration of the assemblies and the lease of 
payloads.

\subsubsection{Polymersomes}

Polymersomes can be considered as an artificial reservoir-like platform, composed of self-assembled amphiphilic copolymers. Typical polymersomes are hollow nanoparticles, similar to the assembly of natural phospholipids, having sizes from about $10 \mathrm{~nm}$ to $1 \mu \mathrm{m}$ range but with a very narrow distribution [90, 368]. In this system, the hydrophobic blocks of the copolymers can aggregate under the aqueous environment, while the hydrophilic blocks would directly confront the inside and outside aqueous solutions, creating two interfaces as the typical bilayered membrane in vesicles produced by micro-phase separation. The vesicle properties, such as size and membrane wall thickness, can be controlled by the copolymer moiety, composition and molecular weight for specific biomedical applications [350]. Hydrophilic drugs can be encapsulated in their aqueous cavities, whereas the hydrophobic component of the membrane can also incorporate hydrophobic drugs. Thus, the format of polymersomes has the ability to deliver hydrophilic and hydrophobic drugs simultaneously to generate synergistic effects to treat tumors.

Recently, polymersomes have attracted a great deal of attention as versatile carriers for biomedical application due to their colloidal stability, tunable membrane thickness and permeability, and ability to encapsulate a broad range of drugs. The relatively long blood circulation times can also be achieved by the introduction of a hydrophilic non-fouling surface layer (e.g., zwitterionic 
materials or PEG). Additionally, the diverse biodegradable and stimuli-responsive polymersomes can be fabricated from the copolymers that are responsive to $\mathrm{pH}$, temperature, redox, light, magnetic field, ionic strength and concentration of glucose [369].

\subsubsection{Polymeric nanogels}

Polymeric nanogels are another promising drug delivery vehicles with unique features including tunable chemical and three-dimensional physical structure, good stability, excellent drug loading capability, and responsiveness to the environmental factors, such as $\mathrm{pH}$, temperature, redox and ionic strength [213, 214, 370-372].

Various LMW biological agents can be loaded into polymeric nanogels through physical interactions, e.g., electrostatic and hydrophobic interactions, between the agents and the polymer matrices. Aggregations and rapid in vivo clearances of nanogels can be prevented by the coating of biocompatible hydrophilic polymers (e.g., PEG) on the surface of drug-loaded nanogels, which forms a protective hydrophilic layer to prevent phase separation. A diverse range of polymeric nanogels have been developed that can deliver their payloads across the different biological barriers and inside the cells $[170,213,370,371]$. Such nanogels generally exhibit high stability and can protect the LMW drugs from degradation due to the metabolic systems of cells.

\subsubsection{Others}


Polymeric nanoparticles, nanocapsules and nanofibers are other commonly exploited nanoscale platforms that can enable the controlled delivery of LMW drugs. Nanoparticles and nanocapsules are both stable solid colloidal systems with diameters in the range of $10-1000 \mathrm{~nm}$ [369]. Nanoparticles are matrix-like vehicles, in which the LMW drugs are dispersed between the polymer chains. Nanocapsule is a vesicular system, which is formed by a drug-containing aqueous core or a lipophilic liquid core surrounded by the polymeric membrane. Polymeric nanoparticles and nanocapsules offer a very wide range of possibilities for modification (e.g. through composition and surface) that can modulate the circulation time, drug loading content and release behavior. The therapeutic applications of nanoparticles and nanocapsules are thus diverse, including cancer therapeutics, antimicrobial action and vaccine delivery, just to name a few. In addition, the polymeric nanoparticles and nanocapsules tend to accumulate in tumors, inflammatory or infectious sites, thereby exhibit the EPR effect on the vasculature [90, 358].

Polymeric nanofibers are referred to polymeric fibers with diameters in the range of $1-1000 \mathrm{~nm}$. The nanoscale diameters of fibers bestow them with unusual properties, including very high surface areas, and enhanced physical, chemical and mechanical properties [373]. Several techniques have been demonstrated that can fabricate polymeric nanofibers, such as electrospinning, self-assembly, template synthesis, and phase separation. Similar to nanoparticles, the polymer matrix in nanofibers can also incorporate LMW drugs, where the delivery can be accomplished from the fibrous scaffold with high surface area, good mechanical strength and adjustable porosity. Several nanofiber-based 
drug delivery systems have been demonstrated for the delivery of anticancer, antibiotics, antifungal, antimicrobial and antihypertensive drugs [373-376].

\subsubsection{Delivery of peptides/proteins}

Recently, peptides/proteins have emerged as promising agents for the therapeutics of various diseases, including cancers, diabetes, anemia, heart attacks, strokes, cystic fibrosis, hemophilia and etc., due to their specific modes of actions and relatively low doses for therapeutic effects [377]. Diverse routes of administration, such as oral, buccal, transdermal, nasal, pulmonary and intravenous pathways, have been demonstrated for the delivery of therapeutic peptides and proteins. However, the controlled delivery of these agents to the targeting sites are often challenged by some unfavorable properties, including large molecular size, susceptibility to denaturation and degradation, short half-life, and poor bioavailability [377, 378]. To solve the above problems, two types of strategies, i.e., chemical conjugation and physical entrapment, have been demonstrated for the efficient delivery of peptides and proteins.

\subsubsection{Chemical conjugation}

Chemical modifications of peptides/proteins with hydrophilic polymers, such as PEG and poly(oligo(ethylene glycol) methacrylate) (POEGMA), have proven effective in enhancing the enzymatic stability, minimizing immunogenicity, as well as improving the circulation time, 
pharmacokinetics and pharmacodynamics [378]. The PEGylated peptides/proteins are usually prepared by the integration of various functional groups in PEG toward primary $\varepsilon$-amines in the lysine residues, thiolates in the reduced cysteine residues, or carboxylic acids in the glutamic and aspartic acid residues of the peptide and protein molecules. To simplify the purification of PEG-peptide/protein conjugates and enhance the modification process, polymerization of POEGMA with predefined length is often performed from the functionalized $N$ or $C$-terminus of a peptide/protein $[379,380]$. This approach has proven to be an efficient means for clinical applications, where several bioactive enzymes, cytokines, hormones and growth factors have been translated to the market or approved for clinical trials (Table 4).

Table 4.

\subsubsection{Physical entrapment}

Physical entrapment is another way to combine therapeutic peptides/proteins with polymeric nanocarriers, such as micelles, vesicles, nanogels, nanoparticles and nanocapsules. This approach has also been proven to be an efficient and secure means for the controlled delivery of peptides and proteins [377], as polymeric nanocarriers can be fined tuned regarding the surface properties and nanoscale structures for the targeted applications. Generally, upon administration, the polymeric nanocarriers should protect the peptide/protein molecules under degradative conditions, such as the presence of enzymes and acidic $\mathrm{pH}$. In addition, the viable peptide/protein formulation should be 
considered not only on the stability, but also on the capability to maintain the native structure and activity during the preparation, storage, administration and delivery. Overall, the application of polymeric nanocarriers for peptide/protein delivery is an emerging field with increasing importance for clinical applications.

\subsection{Gene carriers}

Gene therapy holds great promise for the prevention or treatment of certain diseases and genetic disorders by delivering therapeutic nucleic acids into the defective cells, thereby adjusting and controlling the corresponding cellular processes and responses [393-395] (Fig. 9). The delivery of therapeutic nucleic acids into cells is one of the major hurdles for the successful gene therapy [396, 397]. In other words, therapeutic nucleic acids ( $p$ DNA or siRNA) need to be shuttled and successfully transferred into the defective cells by gene carriers [398]. One way to deal with this problem is through viral based gene carriers. Although viral gene carriers are efficient, the clinical application based on this approach is restricted by several safety concerns, including immunogenicity, carcinogenicity, immune response, and virus replication $[399,400]$. Some of these shortcomings may be overcome by applying non-viral carriers [401, 402]. Polymeric gene carriers have exhibited some unique advantages, such as safety, physiological stability and suitable for large-scale production. However, the transfection efficiency of the non-viral carriers is usually lower than that of the viral carriers. Generally, the complexes that the non-viral carriers form with nucleic acids for gene therapy 
can be classified into four categories: polyplex, lipoplex, micelleplex and others [403, 404].

Fig. 9.

\subsubsection{Polyplex based gene carriers}

Polyplexes are formed by electrostatic interactions between cationic polymers and anionic nucleic acids. The most often used cationic polymers for gene therapy include polyethylenimine (PEI), poly(L-lysine) (PLL), polyamidoamine (PAMAM) dendrimer, chitosan, poly(beta-amino esters), helical polypeptides and cationic aliphatic polyesters. Among these polymers, linear and branched PEIs have received the most attention [398, 400, 405, 406]. For example, PEI25k $\left(M_{w}=25\right.$, 000) has become the gold standard of polymeric gene carriers, which exhibited high transgene efficacy, but its application is also hampered by its relatively high toxicity. Although the low molecular weight PEI (OEI) shows low cytotoxicity, its utility is also limited by the low transgene efficacy [402]. To solve the above problems, Wen and Dong reported polyplexes formed by biodegradable OEI-grafted-polypeptide derivatives and nucleic acids. The in vitro study showed the system exhibited low toxicity but good transgene efficiency [407, 408]. The modification of PEI25k with biocompatible molecules could also improve the transgene efficacy and biocompatibility [398]. In other cationic polymers, PLL was the first cationic polypeptide used for gene therapy [409]. The complexes formed by PLL and nucleic acids with sizes around $100 \mathrm{~nm}$ could be easily endocytosed by cells [410]. However, the application of PLL has been limited due to its poor biocompatibility and 
low transfection ability. To overcome this problem, Kataoka et al. demonstrated PEG- $b$-PLL block copolymers having the improved transfection efficiency due to the incorporation of PEG [411]. To enhance the endosome escape of therapeutic genes during gene transfection, Kataoka et al. also designed an A-B-C triblock copolymer system [412], in which each block possesses a different effect: the PEG block to reduce the toxicity, the low $\mathrm{p} K_{a}$ amine block based on poly[(3-morpholinopropyl) aspartamide] (PMPA) to enhance the endosome escape, and the high $\mathrm{p} K_{a}$ block of PLL to enable DNA binding. Other cationic polymers demonstrated for gene therapy include PAMAM dendrimer due to its relatively high transfection efficacy [413], and polysaccharide, especially chitosan, for its low toxicity as viable non-viral gene carriers for gene therapy [414]. Recently, some relatively new types of biodegradable cationic polymers such as poly( $\beta$-amino esters), helical polypeptides and cationic aliphatic polyesters have been drawn more attention. Poly( $\beta$-amino esters) can condense plasmid DNA into smaller and stable nanoparticles and help to promote cellular uptake and endosomal escape [415-417]. Gene carriers based on $\alpha$-helical polypeptides having the similar properties like cell-penetrating peptides, can effectively deliver genes [418-420]. Aliphatic polyesters representing a new type of biodegradable cationic block copolymer, which has well defined structure and tertiary amine-based cationic groups can effective deliver gene despite high levels of serum [421].

\subsubsection{Lipoplex based gene carriers}


Lipoplexes are widely used for nucleic acid delivery due to their effectiveness and safety [422,

423]. Lipoplexes can be divided into several categories: cationic lipolexes, anionic lipolexes and neutral lipoplexes, according to the type of the charge. Cationic lipoplexes are formed by electrostatic interactions of cationic lipid and anionic molecules (e.g., anionic lipid or anionic nucleic acid). The cationic lipids are usually consisted of a cationic head, linker and hydrophobic segment. In this system, the cationic lipid N-[1-(2,3,-dioleyloxy)propyl]-N,N,N- trimethylammonium chloride (DOTMA) was first introduced for gene delivery by Felgner et al. in 1987, where the mixture of DOTMA and neutral lipid dioleoylphosphatidlethanolamine (DOPE) was used as the gene carrier [423]. Since then, a large variety of cationic lipids have been designed and synthesized as candidates for effective gene therapy [424]. To achieve successful in vivo applications, the lipophilic cationic system must have the capacity to remain in circulation for a prolonged period of time, thus enhancing the opportunity for lipolexes to reach the targeted tissues. However, their potential toxicity is a major obstacle for gene delivery. In order to overcome the toxicity issue, PEG segments are often incorporated into lipoplexes, where PEG can cover the positive charged surface thus improving the biocompatibility [425]. It has been found that the delivery of siRNA may yield a faster result than the delivery of plasmid DNA because the faster diffusion of siRNA in cytosol. We note that DOTMA was first used as a cationic lipid carrier for the RNA delivery [426]. Since then, many cationic RNAi lipoplexes were developed and some are under clinical trials [427]. As the single use of anionic or neutral lipids is not so effective for gene therapy in human [428], neutral lipids are used 
as assistant transgene enhancers in the cationic lipoplex formation.

\subsubsection{Micelleplex based gene carriers}

Micelleplexes are formed by polyion complexation between the phosphate groups in anionic nucleic acids and cationic copolymers with segments that are both cationic and hydrophilic [429, 430]. For example, micelleplexes can be formed by self-assembly of amphiphilic copolymers containing PEG blocks [430]. Micelleplexes are promising candidates for the controlled delivery of nucleic acids because of their tunable characteristics, especially the particle size [430]. Kunath et al. demonstrated that the typical size of micelleplex particles formed by PEGylated copolymers are smaller than those formed by copolymers containing shorter blocks [431]. Recently, to improve the site-specific gene delivery capability, micelleplexes equipped with target moieties were developped, e.g., lactose-equipped micelleplexes for hepatocyte targeting [412]. Recently, micelleplexes for co-delivery of antitumor drugs and therapetic nucleic acids have also been demonstrated [432-438].

For example, Zheng reported that polypeptide cationic micelles can be used for synergistic tumor therapy by co-delivery of docetaxel and therapeutic siRNA [432]. Wang built cationic core-shell nanoparticles using biodegradable amphiphilic copolymer as carrier for co-delivery of paclitaxel with DNA or RNA in therapy of breast cancer in vitro and in vivo [439]. Garbuzenko prepared nanoscale-based delivery system containing doxorubicin and mRNA to treat lung cancer by using inhalation delivery method to enhance accumulation of therapeutic drug in the lungs [440]. Chen 
designed biogradable cationic polylactide nanocapsules to achieve drug-gene co-delivery for prostate cancer by overcoming multidrug resistance [441].

\subsubsection{Others}

Other promising polymer based nanostructured materials for biomedical applications include polymer nanoparticles, cationic nanogels and polymer-modified inorganic particles [193, 442-444]. PLA and PLGA are commonly used to fabricate non-ionic nanoparticles because of their good biocompatibility and degradability. The nucleic acids can be encapsulated in these nanoparticles (PLA or PLGA) by the solvent evaporation or spay-drying method [442]. Nanogels can be surface-functionalized to achieve high loading capacity and tumor-specific targeted delivery due to their high porosity [193]. Taking into account of all the charateristics mentioned earlier, nanogels are very suitable for utilization as gene carriers. The polymer-modified inorganic nanoparticles have also been used in gene therapy for their unique characteristics, such as good biocompatibility, tumor-specific targeting capability, rich functionality and good storage stability [443]. Among this system, the most notable platform is polymer-modified gold nanoparticle [444-446].

Although polymeric non-viral carriers have exhibited great potential for gene delivery, their high toxicity and low transgene expression need to be further improved for clinical applications.

\subsection{Bioimaging}


Bioimaging is a powerful technique that can directly "observe" normal and abnormal biological

processes in individual patients. Many bioimaging modalities have been developed, tested and utilized in preclinical and clinical applications in the past two decades. However, the applications of this technique are often hampered by the poor sensitivity, specificity, and targeting ability of the available and suitable bioimaging probes. The typical polymeric nanostructured bioimaging probes are nanoassemblies consisting of a bioimaging core and a polymer coating as shell. The polymer coating not only protects the loaded probes from the environment, but also improves the pharmacokinetics and bio-distribution of the probes, thus significantly amplifying the diagnostic imaging signals. The developed modalities include contrast agents for magnetic resonance imaging (MRI), X-ray computed tomography (CT), fluorescence imaging (FI), single-photo emission computed tomography (SPECT) and positron emission tomography (PET), which will be discussed as follows.

\subsubsection{Magnetic resonance imaging}

Magnetic resonance imaging (MRI) is a powerful technology to visualize the internal structures of the body (e.g. brain, muscle, heart, and cancer regions) using magnetic fields and radio waves. This technology generally provides superior contrasts between the different soft tissues of the body compared to other imaging modalities. However, the inherent contrast in some parts of the body is often insufficient for clear differentiation, e.g., detection of small tumors. To overcome this problem, 
one must rely on the use of MRI contrast enhancing agents. The most commonly used MRI contrast agents are low molecular weight chelates of gadolinium $\left(\mathrm{Gd}^{3+}\right)$ or iron oxide particles. Often, the clinical applications of these MRI contrast agents are limited by the short imaging time window, low signal to noise ratio (as a result of the short transient tissue retention time), toxicity, and unfavorable pharmacokinetic profiles. Polymer based nanotechnology has exhibited great potentials to reduce the toxicity of metal ions, prolong the blood circulation time of the agent/probe, and improve the contrast. This is because the suitable polymer system can connect the specific ligands on the probe.

Biocompatible polymers such as polylysine, poly(L-glutamic acid)-cystamine, poly(ethylene glycol), poly (lactic acid)-poly(ethylene glycol)-poly(l-lysine), poly(ethylene glycol)-b-poly(N-(N'-(2-aminoethyl)-2-aminoethyl)aspartamide), polysilsesquioxane, dextran, and L-cystine bisamide copolymer have been used for the preparation of MRI contrast enhancing agents through conjugation with low-molecular weight Gd chelates [447-450]. Bock et al. reported that the use of Gd-DTPA-polylysine could increase the signal intensity by $118 \%$ in pulmonary arteries of healthy lungs and by $121 \%$ in damaged lungs [451]. The PEGylation strategy in PLL has also been used to modulate the pharmacokinetic properties of the MRI contrast agents. For example, Yokoyama et $a$. found that the usage of PEG- $b$-poly(L-lysine) could significantly prolong the circulation time of gadolinium ion in blood. A considerable amount of PEG-P(Lys-DOTA-Gd) micelles was found to accumulate in solid tumors $24 \mathrm{~h}$ after intravenous injection due to the EPR effect. As a result, the MRI signal intensity of the tumor was enhanced 2.0-fold by the use of this 
polymeric micelle contrast agent [452]. Na and coworkers also reported a cancer-recognizable MRI contrast agents (CR-CAs), consisting of methoxy poly(ethylene glycol)-b-poly(L-histidine) (PEG-p(L-His)) and methoxy poly(ethylene glycol)- $b$-poly(L-lactic acid)-diethylenetriaminopentaacetic acid dianhydride-gadolinium chelate (PEG-p(L-LA)-DTPA-Gd) [453]. The CR-CAs had a spherical shape with a uniform size of $\sim 40 \mathrm{~nm}$ at the physiological $\mathrm{pH}$ (pH $=7.4)$ level. Under the acidic tumoral environment $(\mathrm{pH}=6.5)$, the CR-CAs disintegrated into positively charged water-soluble polymers due to the protonation of the imidazole groups of $\mathrm{p}(\mathrm{L}-\mathrm{His})$ segments. As a result, the CR-CAs exhibit highly effective T1 MR contrast enhancement in the tumor region, which enabled the detection of small tumors of $\sim 3 \mathrm{~mm}^{3}$ in vivo at $1.5 \mathrm{~T}$ within a few minutes (Fig. 10).

Fig. 10

Dendrimers such as Gadomer, PAMAM and polypropylenimine tetrahexacontaamine, have also been used for the conjugation with low-molecular weight $\mathrm{Gd}$ chelates [454-459]. The unique advantages of using dendrimers include the uniform surface chemistry, monodisperse molecular weight and shape. In particular, dendrimers have a large number of surface amino groups in a compact spherical space. With these groups, one can significantly enhance the MRI signals by attaching low molecular weight based chelates of gadolinium, such as Ga-DTPA. For example, Kobayashi et al. demonstrated that the conjugates of DAB-G5 and PAMAM-G8 with chelate of Ga 
could successfully be used to identify lymph nodes and lymphatic vessels, respectively [454].

In the past two decades, the use of superparamagnetic iron oxide nanoparticles for medical imaging has advanced notably. Based on the particle size, iron oxide nanoparticles for MRI can be classified into superparamagnetic iron oxide (SPIO, diameter $>50 \mathrm{~nm}$ ) and ultrasmall superparamagnetic iron oxide (USPIO, diameter $<50 \mathrm{~nm}$ ). SPIO agents can be used to image tumors in the liver and spleen, while USPIO agents are often used to image of lymphograpy and angiography [447, 460, 461]. Superparamagnetic iron oxide nanoparticles can enhance the negative contrast in MRI by causing tissues to appear darker than their surroundings because of their very large $\mathrm{T} 2$ relaxation and negligible $\mathrm{T} 1$ relaxation. However, as natural iron oxide particles have the following problems: easy aggregation, change in magnetic properties, rapid degradation under physiological conditions, and toxicity, they cannot be directly used in clinical applications. To overcome these problems, SPIO and USPIO contrast agents in clinical and preclinical applications are usually coated with polymers [462], such as dextran [463, 464], dextran derivatives [463, 464], silicone [463], oxidized-starch [464], PVA [465], or PEG [466]. Briley-Saebo et al. investigated the effects of the particle size and the coating material based on the various iron oxide nanoparticles on the rate of liver clearance in rat. They found that materials with similar coating but different sizes exhibited similar rates of liver clearance, but the coating material could significantly alter the rate of iron oxide clearance in rat liver. For example, the half-life of iron oxide nanoparticles in rat liver was 8 days for dextran-coated materials, 10 days for carboxydextran materials, 14 days for unformulated 
oxidized-starch, and 29 days for formulated oxidized-starch [464]. Several long-circulating dextran or carboxydextran coated iron oxide nanoparticles, including Feridex/Endorem (Ferumoxide), Resovist/Cliavist (Ferucarbotran), and Combidex/Sinerem (Ferumoxtran) have already been approved for clinical usage [467].

\subsubsection{X-ray computed tomography}

X-ray computed tomography (CT) has become a common tool in the arsenal of modern diagnostic medicine [468, 469]. Unenhanced X-ray imaging provides superior visualization of bone structures due to the inherent contrast between electron-dense bones and permeable surrounding soft tissues. However, the native contrast between the different soft tissues is so small that unenhanced X-ray imaging cannot differentiate between them. To enable better delineation of soft tissue regions such as the cardiovascular system, CT contrast agents were introduced [470, 471]. Contrast agents used in CT contain atoms that are electron dense, such as barium [472], iodine [473], bismuth [474, 475], lanthanide and gold [476, 477]. The CT contrast is linearly proportional to the total amount of the high- $Z$ molecules in a voxel. Thus, in order to induce sufficient contrast in the desired organ, a much larger amount of contrast agent molecules is needed [471].

Small molecular weight iodine compounds are dominant CT contrast agents in the present clinical applications. However these iodinated molecules have very short imaging times due to the rapid clearance by the kidneys [471], which could significantly restrict the applications of CT in 
target-specific imaging and angiography [469]. In addition, a large dose of iodines, which are often used to enhance the imaging because of their low contrast efficacy, can lead to serious adverse effects (e.g., some patients are hypersensitive to iodine [469]). To address these problems, functional polymeric nanoparticles have been developed [478-480]. For example, Seo and coworkers reported that lipiodol-encapsulated Pluronic/PEG-crosslinked nanocapsules had stable structure at high concentrations and with a safety profile similar to or better than that of Iopromide. These polymeric nanoparticles exhibited a longer circulation time than the commercial iodinated system [481]. However, the contrast efficacy of the iodinated nanocapsules is still relatively low.

To enhance the contrast efficacy, a variety of metal-based nanoparticulate contrast agents have been explored, besides iodinated agents [477]. Among these, gold nanoparticles (AuNPs) have received the most interest as CT contrast agents. This is because they can be readily functionalized with small molecules [482], PEG [483] or targeted proteins [484]. For example, Aurovist ${ }^{\circ}$, a commercial product of AuNPs with thioglucose coating, has recently been approved in Europe for imaging applications [485]. Generally, functionlized AuNPs show a prolonged blood circulation time, low toxicity, and comparable or better efficacy against iodinated agents [486-489]. Lu et al. also reported a binary contrast agent BaYbF5@SiO2@PEG that exhibited a long retention time in vasculature, remarkably low cytotoxicity and much higher contrast efficacy than Iobitridol and NaYbF4@PEG with a single contrast element [490]. The general challenges for the development of new and effective CT contrast agents include the route of functionalization, in vivo targeting, 
efficiency, long-term stability, toxicology and excretion [469].

\subsubsection{Fluorescence imaging}

Fluorescence imaging has been widely used for non-invasive studies of fundamental mechanisms and processes at the organ, tissue, cellular, and molecular levels. Because tissue is relatively transparent for near-infrared (NIR) light, the ideal near-infrared fluorescent (NIRF) agents for in vivo imaging should have a peak fluorescence in the range of 700-900 nm. In addition, the characteristics of high quantum yield, a narrow excitation/emission spectrum, high stability, non-toxicity, available functionality for conjugation, good biocompatibility, good biodegradability and good excretability are important for NIRF imaging agents [491-493]. Organic NIRF agents such as indocyanine green (ICG) and inorganic fluorescent agents such as quantum dots (QDs) fit some of the above specifications. However, they also have some limitations, such as a lack of specificity for their targeted cells, tissues or organs, rapid aggregation, and short blood circulation time [447]. Polymeric fluorescent agents can prevent rapid aggregation, prolong the blood circulation time, improve stability, enhance targeting capabilities and reduce background signals. A variety of polymer-based protease-activatable or targeted imaging probes have been developed and demonstrated good fluorescence imaging capability [447, 494-497]. Some examples are as follows.

It is well known that PLL backbone is cleavable by protease such as cathepsin B and trypsin [498, 499]. A polymer-based protease-activatable imaging probe (Cy5.5) ${ }_{11}-\mathrm{PLL}_{-\mathrm{mPEG}}$ has been 
successfully developed. In (Cy5.5) $)_{11}-$ PLL- PEG $_{92}$, the PLL backbone contained an average of 44 unmodified lysines, 92 methoxy polyethylene glycol side chains and 11 molecules of the near-infrared fluorochrome Cy5.5. The existence of unmodified lysine groups renders the polymeric imaging probe cleavable by cathepsin B and trypsin [500]. The in vitro evaluation of this system showed that the enzyme-activated form of (Cy5.5) ${ }_{11}-$ PLL-mPEG $_{92}$ exhibited 12-fold higher NIR fluorescence signals than the unactivated probes [498]. Such polymer-based protease-activatable imaging probes can be used for the in vivo detection of cathepsin B-overexpressed diseases such as cancer [501, 502], rheumatoid arthritis [503], and atherosclerosis [504]. Using the peptides as protease-activatable linkers grafted on the side groups of polymers, a variety of polymer-based protease-activatable imaging probes could be obtained. The demonstrated proteases include cathepsins [505], matrix metalloproteinases [494], thrombin [506], factor XIIIa [507], caspases [508], urokinase-type plasminogen activator [509], and HIV protease. These polymer-based protease-activatable imaging probes have great potential to be used as effective tools to target a variety of protease $[447,510]$.

Compared with organic fluorophores, quantum dots (QDs) have special advantages for fluorescence imaging, including easy control of excitation and emission wavelengths, high quantum yield, easy modification, and no fluorescent bleaching [447]. However, the high toxicity in vivo and low solubility in water have hindered the clinical applications of QDs [511, 512]. To address the toxicity and solubility problems, QDs were encapsulated by amphiphilic copolymers [513-516]. The 
improved stability and targeting ability, reduced toxicity, and enhanced solubility in aqueous solutions have been achieved through polymer coating [125, 517-520], a process that has been discussed earlier.

\subsubsection{Nuclear imaging}

Nuclear imaging is a technique for producing images of various body parts using radioactive agents that can be traced in the body using a gamma ray camera. The acquired information is not only useful for diagnostic purposes, such as detection of functional abnormalities or early identification of tumors, but also helpful in therapy planning and follow-up procedures. Positron emission tomography (PET) and single photon emission computed tomography (SPECT) are two most commonly used nuclear imaging techniques.

PET is an imaging technique that detects pairs of gamma rays emitted indirectly by a positron-emitting radionuclide introduced into the body. It has been clinically used for oncology, neurology, cardiology and pharmacology. As compared with other molecular imaging modalities, PET has the advantages of high sensitivity (the level of detection can approach $10^{-11} \mathrm{M}$ of tracer) and isotropism (i.e., the ability to detect expression accurately, regardless of tissue depth) [521]. At present, the most commonly used radiotracer in clinical PET scanning is fluorodeoxyglucose (also called FDG or fludeoxyglucose). However, FDG does not have the specific targeting ability as some newer bioimaging probes [447]. ${ }^{64} \mathrm{Cu}$ is another popular radionuclide for PET imaging due to its 
suitable half-life $\left(t_{1 / 2}=12.7 \mathrm{~h}\right)$, appropriate positron emission energy $(0.65 \mathrm{MeV})$, and relatively convenient radiolabeling ability via coordination with specially designed chelators [524]. Several demonstrated schemes to improve of the specific activity of radiopharmaceuticals have allowed the ${ }^{64} \mathrm{Cu}$-based PET system to achieve high quality images at low dosage [523, 524]. One very promising scheme is to encapsulate or conjugate ${ }^{64} \mathrm{Cu}$ chelating agents with nanocarriers [525-527]. With this approach, Wooley et al. developed shell crosslinked (SCK) nanoparticles termed ${ }^{64} \mathrm{Cu}$-complexed pre-DOTAlysine-SCK, based on the copolymer of poly(acrylic acid)- $b$-polystyrene grafted with DOTAlysines. The SCK nanoparticles showed impressive specific activity (ca. $400 \mu \mathrm{Ci}$ $\mu \mathrm{g}^{-1}$ ), suggesting that these nanoparticles might be used for the development of highly sensitive in vivo PET tracers at low administering dosage [521].

Similar to PET, SPECT uses radiotracer (that emits gamma radiation) and scanner/detector to construct two- or three-dimensional images of the body part of interest. Generally, SPECT is cheaper than PET and the technique has also been clinically used for tumor imaging, infection imaging, thyroid imaging and bone scintigraphy. Gamma emitters, such as ${ }^{99} \mathrm{Tc},{ }^{111} \mathrm{In}$, or ${ }^{166} \mathrm{Ho}$, are common radiotracers [528-530]. The low molecular weight radiotracers have short circulation times, which often do not allow the agents to successfully penetrate into the targeted tissue. As a result, the sensitivity of nuclear imaging can be greatly compromised by the existing radiotracers. However, this problem can be overcome by using tumor targeting ligand decorated nanoparticles with long circulation times as the carriers of radionuclide. For example, Li et al. reported that PC-3M tumors 
could be clearly visualized by SPECT after intravenous administration of ${ }^{111}$ In-labeled core-crosslinked polymeric micelle nanoparticles with EphB4 binding peptide [531].

\subsection{Tissue engineering and regenerative medicine}

Nanotechnology and nanoengineering are effective means in the design, preparation, characterization and applications of nanoscale devices, which consist of functional organizations with at least one dimension in the range from several to hundreds nanometers. Recently, the synergy between nanoscience and tissue engineering has lead to great developments in biomedical research as well as clinical practices, including the realms of bone and cartilage regeneration, blood vessel tissue engineering, wound dressing, and so on.

\subsubsection{Bone tissue engineering}

Bone tissue engineering is an important branch of tissue engineering with aims to repair and/or regenerate bone tissue using cell-based therapies and/or growth supplements based on functional scaffolds. This technology is usually used to restore the skeleton function in the process of orthopedic or oral-maxillofacial surgery [532]. In this approach, scaffolds are considered as the fundamental element for the success of engineered constructs, which are developed to mimic the biophysical structures of nature extracellular matrices (ECMs) and to provide an appropriate microenvironment for the host-cell colonization. These scaffolds are often porous biodegradable 
three-dimensional (3D) structures that can meet some fundamental properties of ECMs such as biocompatibility, osteoconductivity, osteoinductivity, osteogenicity and mechanical match between the implanted scaffold and surrounding tissues [533, 534].

Various scaffolds have been prepared using recently developed nanofabrication techniques that can endow us the capability to modulate aperture configuration and nanostructures. In bone, the organic matrix (that amounts to about $85 \%$ of the mass) is made of native collagen type I fibrils, which are approximately $50 \mathrm{~nm}$ in diameter. The aligned or irregular patterns of these fibrils induce the distinguishing characteristics of lamellar or woven bone $[535,536]$. In view of the above characteristics, polymeric nanofibers of similar diameters can be uniquely fabricated to imitate the fibrous nature of bone ECMs in bone engineering.

Several in vitro and in vivo studies have confirmed the possibility of using polymeric nanofibers as scaffolds for bone regeneration. Three specific approaches, i.e., electrospinning [228, 537], phase separation [538] and self-assembly [539], are commonly used to produce polymeric nanofibers for tissue scaffolding [535]. As a typical example for bone tissue engineering, PCL scaffolds containing electrospun nanofibers with dimensions from $20 \mathrm{~nm}$ to $5 \mathrm{~mm}$ have been employed to support the in vitro mineralization and differentiation of bone marrow-derived mesenchymal stem cells (BM-MSCs) from rat [540]. It was found that the PCL scaffold with nanofiber diameter $~ 370 \mathrm{~nm}$ was particularly effective, where the scaffold could facilitate both adhesion and proliferation of MSCs and create higher levels of alkaline phosphatase activity, mineralization, and osteocalcin and osteopontin 
productions. The effectiveness of the PCL nanofibrous scaffold was further evaluated through an in vivo study. In this study, the scaffold was seeded with MSCs and subsequently implanted in the omenta of rat for 4 weeks [541], where the cells were found to successfully differentiate and infiltrate into the scaffolds [542]. In addition, nanofibers based on self-assembled peptide-amphiphiles (e.g., Arg-Gly-Asp (RGD) and phosphoserine) were found to be able to promote the cellular binding and mineralization [543]. Composite polymeric nanofibers containing osteinductive factors have also been prepared [535, 544] for bone engineering. For example, nanofibers consisting of silk, PEG, hydroxyapatite nanoparticles and bone morphogenetic protein 2 (BMP-2) were electrospun to create composite scaffolds. In these scaffolds, the silk/PEG nanofibers were found to be capable of supporting the osteogenic differentiation of human MSCs (hMSCs), where the presences of BMP-2 and hydroxyapatite nanoparticles could significantly enhance the bone formation in vitro [545].

Polymeric nanoparticles have also been used for bone tissue engineering. These materials are mainly to entrap and deliver biomolecules (growth and differentiation factors), bone morphogenetic proteins and genetic materials [546, 547]. As polymeric micelles, polymersomes, nanogels, nanoparticles, nanocapsules and dendrimers are all possible vehicles for controlled delivery, their usage for bone regeneration requires some specific considerations. In general, the solid, hollow or porous nanoparticles are suitable for bone applications. These materials can be prepared through self-assembly, nanomanipulation, bioaggregation and photochemical patterning [547-549]. The scaffolds for bone tissue engineering containing bioactive polymeric nanoparticles can exhibit 
several advantages over traditional monolithic scaffolds: (1) enhanced control over sustained delivery of therapeutic agents; (2) acting as porogen or reinforcement phase to introduce porosity and/or improve the mechanical properties of bulk scaffolds; (3) acting as compartmentalized microreactors for dedicated biochemical processes; (4) acting as cell delivery vehicles; and (5) imbedding injectable or moldable formulations to be applied in minimally invasive surgery [550].

\subsubsection{Cartilage tissue engineering}

Articular cartilage covers the articular surface and protects the underlying bone. The tissue in articular cartilage consists of chondrocytes, collagen (primarily type II), proteoglycans and water, where the ECMs play an essential role in both biological and mechanical functions in this tissue [532]. Thus, a well-designed scaffold that can mimic the structure and functions of native ECMs is highly desired for the development of engineered cartilage. Most ECMs are in the form of a mesh structure containing fibrils that provides tensile mechanical properties and also traps bioactive molecules in the fibrous network $[535,551]$. This fibrous structure of polymer nanofibers thus becomes an ideal scaffold for engineered articular cartilage.

In cartilage tissue engineering, polymeric nanofibrous scaffolds were found to be able to prevent de-differentiation and promote re-differentiation of chondrocytes. For example, when fetal bovine chondrocytes were cultured in a chondrogenic growth media on the PCL nanofibrous scaffold (fiber diameter about $700 \mathrm{~nm}$ ), higher levels of proliferated and expressed cartilage-associated genes were 
achieved than those cultured on the polystyrene platform typically used in tissue culture [552]. In addition, bovine articular chondrocytes were found to be capable of infiltrating the PCL nanofibrous scaffolds having diameters ranging from 400 to $1400 \mathrm{~nm}$ [553]. These results indicated the great potential of using polymeric nanofibrous constructs as scaffolds for the support of chondrocytes in vitro and in vivo. Polymeric nanofibrous scaffolds have also been exploited to sustain the chondrogenesis of progenitor cells. For instance, electrospun PCL scaffolds with fiber diameters of approximately $700 \mathrm{~nm}$ were shown to crutch multilineage differentiation of BM-hMSCs [554]. The level of chondrogenesis on the nanofiber scaffolds was shown to be equivalent and even higher than the gold standard pellet cultures in some cases. In addition, polymeric nanofiber scaffolds could improve mechanical properties, making them a viable option for in vivo transplantation [555]. Recently, an in vivo study demonstrated that the implantation of hMSCs in the nanofibrous PCL scaffolds to a swine model could induce the formation of hyaline-like cartilage with a smooth cartilage surface [535].

Similar to bone tissue engineering, polymeric nanoparticles have also been used to deliver growth factors in cartilage regeneration therapies. Although polymeric nanoparticles can be shaped into different platforms to control the release of growth factors, most of the nanoparticle systems for cartilage regeneration are designed for local delivery, often through the pathways of hydrogels and composite constructs $[547,554,556]$. The incorporation of polymeric nanoparticles as dispersing agents in tissue scaffolds would often induce nanostructured features on the scaffold surface, which 
can improve cellular adhesion and other cellular behaviors.

\subsubsection{Vascular tissue engineering}

Vascular tissue engineering is a means to substitute large-scale blood vessels with diameters greater than $6 \mathrm{~mm}$, where the procedure would induce microvasculature or neovascularization processes inside or near the implanted scaffolds. To achieve good blood vessel regeneration, various vascular tissue scaffolds, including nanoscale porous membranes (e.g., electrospun polymeric nanofibrous scaffolds) have been designed and fabricated for different types of blood vessels. Some specific considerations for the fabrication of these scaffolds are: (1) they should exhibit proper mechanical strength and elasticity; (2) they should maintain endothelial coverage to control the diverse physiological signals; and (3) the remodeling of blood vessel should be able to respond stimulatory cues (Fig. 11) [557].

Fig. 11

In the past decades, notable efforts have been made to produce vascular scaffolds with nanoscale properties, aiming to replicate the ECMs architecture. ECMs consist of nanofibers with diameters in the range of $5-500 \mathrm{~nm}$, and compositions including collagens, elastins and nanoscale adhesive proteins (e.g. laminin and fibronectin) $[558,559]$. Thus, the typical engineered scaffolds are 3D constructs having porous interwoven structures. For example, Zhang and coworkers have 
demonstrated a nanostructured polymeric scaffold (with the feature dimension between $200-400$ $\mathrm{nm})$, which exhibited excellent mechanical properties and stable tube-like networks for cell seeding [560].

The incorporation of bioactive polymeric nanoparticles into the scaffolds is a logical strategy to enhance the regenerative capacity of tissue-engineering devices. In blood vessel regeneration, polymeric nanoparticles can be used for this purpose and deliver bioactive biomolecules such as adhesion molecules, growth factors, extracellular matrices, tight junction proteins and signaling molecules. These molecules can penetrate into the microvasculature inside the tissue scaffolds, cells or cell nuclei $[557,561]$ and accelerate the regeneration process.

\subsubsection{Wound dressing}

The wound dressing is developed to prevent further harm, promote healing and achieve the best aesthetic repair [562]. Electrospun polymeric nanofibers are among the most advanced and efficient wound dressing materials with performance that can surpass other existing wound dressing materials such as hydrocolloids, hydrogels, and alginates. This is because the bandage made of non-woven polymeric nanofibers is a natural 3D porous architecture with high surface area. The porous nature of nanofibrous scaffolds enable excellent ability to absorb wound exudates, prevent the moisture loss around the wound, allow oxygen permeation, protect the wound from bacterial infection, and exhibit good conformability [562, 563]. A diverse range of synthetic and natural polymers, including 
polyurethane (PU), PLA, PCL, PLGA, polyvinyl alcohol, dextran, chitin, chitosan, cellulose acetate, gelatin and collagen, have been exploited as candidates for dressing materials, where bioactive agents such as anti-inflammatory drugs and tissue growth agents were also incorporated in the polymeric nanofibers for controlled delivery [562, 564-567].

\subsubsection{Others}

Polymeric nanostructured materials, especially polymeric nanofibers, have also been used in other areas of tissue engineering, such as tendon and ligament, neural, and cardiovascular tissue repair [535, 562]. In tendon and ligament tissue engineering, the scaffolds based on aligned polymeric nanofibers have been considered as a promising platform because the scaffold structure has the anisotropy similar to that of native tissue [568]. In addition, polymeric nanofibers are ideal to develop effective neural guidance conduits, suitable for bridging gaps in damaged peripheral or central neurons. Again, this is because the nanofibrous scaffolds have a structure very similar to that in neural ECMs. As a result polymeric nanofibrous conduits can also be used to direct axon sprouting and deliver neurotrophic factors to the site of injury [535]. For cardiac tissue engineering, the polymeric nanofibers can mimic the fiber-liker feature of natural tissue architecture, where the nanofibrous scaffold was found to be effective in inducing elongated and aligned cardiomyocytes [569]. 


\section{Conclusions and future challenges}

In conclusion, efforts to develop polymeric nanostructured materials have attracted a great deal of attention due to the increasingly importance of these materials in biomedical applications. Recent and extensive reports on the development of fabrication methods that can prepare various polymeric nanostructured materials, including micelle, polymersome, nanoparticle, nanocapsule, nanogel, nanofiber, dendrimer and nanocomposite, have been reviewed. Internal material properties, such as solubility, interactions between polymer and payload, polymer chain flexibility, surface charge, stereochemistry, surface chemistry, molecular weight, and crystallization ability, etc. should be taken into consideration for the selection of appropriate preparation method and suitable processing conditions.

Polymeric nanostructured materials (PNM) can provide great value for accurate diagnosis and effective treatment of diseases. For instance, in controlled delivery of bioactive molecules (e.g., low molecular weight drugs and genes), PNMs can be used to enhance the in vivo stability, increase the target specific delivery of drugs and genes, optimize the pharmacokinetics and biodistribution of the payload, reduce the side effects, and improve the efficacy of the system. For bioimaging, polymeric nanostructured contrast agents can exhibit prolonged blood circulation time, thus enhancing the targetability and reducing the adverse effects of toxic probe. Polymer-based nanotechnology can also

provide opportunities for personalized diagnosis and treatment by combining therapeutic and 
imaging contrast agents together. In addition, for tissue engineering and regenerative medicine, polymeric nanofibrous scaffolds can offer the unique advantage of imitating the 3D fibrous porous structure of natural ECMs.

However, though polymeric nanostructured materials have shown great potential to revolutionize the diagnosis and treatment of disease, there are also great challenges for the successful translation of basic research to clinical applications. For example, the issue of large scale production of PNMs must be carefully addressed, especially for those PNMs that require many steps to complete. The toxicity of the materials for cells, tissues and organs are another important issue to consider, especially for those PNMs that contain non-biodegradable or inorganic components. In addition, PNMs and the loaded therapeutic and imaging agents usually have a pharmacokinetics pattern different from the currently used low molecular weight drugs and imaging agents, which must also be investigated. In spite of these challenges, PNMs offer new possibilities to complement or replace the existing systems. We certainly believe that PNMs will become a major diagnostic and therapeutic tool for biomedical applications in the future.

\section{Acknowledgments}

The authors thanks Wantong Song, Xiaoye Gao, Yuce Li, Jie Chen, Xiaofeng Song for the help in the review. This work was supported by National Natural Science Foundation of China (Projects 51173184, 51373168, 21174142, 51222307, 51390484, 51233004 and 51321062), Ministry of 
Science and Technology of China (International Cooperation and Communication Program 2011DFR51090), Jilin Province Science and Technology Development Program (20130206058GX and 20130521011JH). B.H. acknowleges the support from the National Science Foundation (DMR-1409507) in the U.S. 


\section{References}

[1] Chen M, Yin M. Design and development of fluorescent nanostructures for bioimaging. Prog Polym Sci 2014;39:365-95.

[2] Baba M, Matsumoto Y, Kashio A, Cabral H, Nishiyama N, Kataoka K, Yamasoba T. Micellization of cisplatin (NC-6004) reduces its ototoxicity in guinea pigs. J Controlled Release 2012;157:112-7.

[3] Kim HN, Guo ZQ, Zhu WH, Yoon J, Tian H. Recent progress on polymer-based fluorescent and colorimetric chemosensors. Chem Soc Rev 2011;40:79-93.

[4] Hrkach J, Von Hoff D, Ali MM, Andrianova E, Auer J, Campbell T, De Witt D, Figa M, Figueiredo M, Horhota A, Low S, McDonnell K, Peeke E, Retnarajan B, Sabnis A, Schnipper E, Song JJ, Song YH, Summa J, Tompsett D, Troiano G, Van Geen Hoven T, Wright J, LoRusso P, Kantoff PW, Bander NH, Sweeney C, Farokhzad OC, Langer R, Zale S. Preclinical development and clinical translation of a PSMA-targeted docetaxel nanoparticle with a differentiated pharmacological profile. Sci Transl Med 2012;4:128ra39/1-10.

[5] Hahn MA, Singh AK, Sharma P, Brown SC, Moudgil BM. Nanoparticles as contrast agents for in-vivo bioimaging: current status and future perspectives. Anal Bioanal Chem 2011;399:3-27.

[6] Liu Q, Yang T, Feng W, Li F. Blue-emissive upconversion nanoparticles for low-power-excited bioimaging in vivo. J Am Chem Soc 2012;134:5390-7.

[7] Kamaly N, Xiao ZY, Valencia PM, Radovic-Moreno AF, Farokhzad OC. Targeted polymeric therapeutic nanoparticles: design, development and clinical translation. Chem Soc Rev 2012;41:2971-3010.

[8] Elsabahy M, Wooley KL. Design of polymeric nanoparticles for biomedical delivery applications. Chem Soc Rev 2012;41:2545-61. 
[9] Chan JM, Rhee JW, Drum CL, Bronson RT, Golomb G, Langer R, Farokhzad OC. In vivo prevention of arterial restenosis with paclitaxel-encapsulated targeted lipid-polymeric nanoparticles. Proc Natl Acad Sci USA 2011;108:19347-52.

[10] Cheng Z, Al Zaki A, Hui JZ, Muzykantov VR, Tsourkas A. Multifunctional nanoparticles: cost versus benefit of adding targeting and imaging capabilities. Science 2012;338:903-10.

[11] Shi J, Votruba AR, Farokhzad OC, Langer R. Nanotechnology in drug delivery and tissue engineering: from discovery to applications. Nano Lett 2010;10:3223-30.

[12] Liu W, Thomopoulos S, Xia Y. Electrospun nanofibers for regenerative medicine. Adv Healthc Mater 2012;1:10-25.

[13] Mukherjee S, Venugopal JR, Ravichandran R, Ramalingam M, Raghunath M, Ramakrishna S. Nanofiber Technology for Controlling Stem Cell Functions and Tissue Engineering. Micro and Nanotechnologies in Engineering Stem Cells and Tissues: John Wiley \& Sons, Inc.; 2013. p. 27-51.

[14] Li X, Yang Y, Fan Y, Feng Q, Cui FZ, Watari F. Biocomposites reinforced by fibers or tubes as scaffolds for tissue engineering or regenerative medicine. J Biomed Mater Res A 2014;102:1580-94.

[15] Kumari A, Yadav SK, Yadav SC. Biodegradable polymeric nanoparticles based drug delivery systems. Colloids Surf B Biointerfaces 2010;75:1-18.

[16] Gu Z, Biswas A, Zhao MX, Tang Y. Tailoring nanocarriers for intracellular protein delivery. Chem Soc Rev 2011;40:3638-55.

[17] Hoffman AS. Stimuli-responsive polymers: Biomedical applications and challenges for clinical translation. Adv Drug Del Rev 2013;65:10-6.

[18] Alarcon CDH, Pennadam S, Alexander C. Stimuli responsive polymers for biomedical applications. Chem Soc Rev 2005;34:276-85.

[19] Meng F, Zhong Z, Feijen J. Stimuli-responsive polymersomes for programmed drug 
delivery. Biomacromolecules 2009;10:197-209.

[20] Oh JK, Park JM. Iron oxide-based superparamagnetic polymeric nanomaterials: design, preparation, and biomedical application. Prog Polym Sci 2011;36:168-89.

[21] Liang D, Hsiao BS, Chu B. Functional electrospun nanofibrous scaffolds for biomedical applications. Adv Drug Del Rev 2007;59:1392-412.

[22] Svenson S, Tomalia DA. Dendrimers in biomedical applications-reflections on the field. Adv Drug Del Rev 2012;64:102-15.

[23] Hule RA, Pochan DJ. Polymer nanocomposites for biomedical applications. MRS Bull 2007;32:354-8.

[24] Rezwan K, Chen Q, Blaker J, Boccaccini AR. Biodegradable and bioactive porous polymer/inorganic composite scaffolds for bone tissue engineering. Biomaterials 2006;27:3413-31.

[25] Dash M, Chiellini F, Ottenbrite RM, Chiellini E. Chitosan-A versatile semi-synthetic polymer in biomedical applications. Prog Polym Sci 2011;36:981-1014.

[26] Serrano MC, Gutierrez MC, del Monte F. Role of polymers in the design of 3D carbon nanotube-based scaffolds for biomedical applications. Prog Polym Sci 2014;39:1448-71.

[27] Peer D, Karp JM, Hong S, FaroKHzad OC, Margalit R, Langer R. Nanocarriers as an emerging platform for cancer therapy. Nat Nanotechnol 2007;2:751-60.

[28] Schleich N, Po C, Jacobs D, Ucakar B, Gallez B, Danhier F, Préat V. Comparison of active, passive and magnetic targeting to tumors of multifunctional paclitaxel/SPIO-loaded nanoparticles for tumor imaging and therapy. J Controlled Release 2014;194:82-91.

[29] Lee SJ, Min HS, Ku SH, Son S, Kwon IC, Kim SH, Kim K. Tumor-targeting glycol chitosan nanoparticles as a platform delivery carrier in cancer diagnosis and therapy. Nanomedicine 2014;9:1697-713.

[30] Uzgiris EE. Polymeric Nanoparticles for Medical Imaging. In: Broz P, editor. 
Polymer-Based Nanostructures: Medical Applications. 1st ed: The Royal Society of Chemistry; 2010. p. 173-236.

[31] Deng C, Jiang Y, Cheng R, Meng F, Zhong Z. Biodegradable polymeric micelles for targeted and controlled anticancer drug delivery: promises, progress and prospects. Nano Today 2012;7:467-80.

[32] Parveen S, Misra R, Sahoo SK. Nanoparticles: a boon to drug delivery, therapeutics, diagnostics and imaging. Nanomed Nanotechnol Biol Med 2012;8:147-66.

[33] Letchford K, Burt H. A review of the formation and classification of amphiphilic block copolymer nanoparticulate structures: micelles, nanospheres, nanocapsules and polymersomes. Eur J Pharm Biopharm 2007;65:259-69.

[34] Mourya VK, Inamdar N, Nawale RB, Kulthe SS. Polymeric Micelles: General Considerations and their Applications. Ind J Pharm Edu Res 2011;45:128-38.

[35] Jones MC, Leroux JC. Polymeric micelles - a new generation of colloidal drug carriers. Eur J Pharm Biopharm 1999;48:101-11.

[36] Gao Z, Eisenberg A. A model of micellization for block copolymers in solutions. Macromolecules 1993;26:7353-60.

[37] Alexandridis P, Holzwarth JF, Hatton TA. Micellization of Poly(ethylene oxide)-Poly(propylene oxide)-Poly(ethylene oxide) Triblock Copolymers in Aqueous Solutions: Thermodynamics of Copolymer Association. Macromolecules 1994;27:2414-25.

[38] Ma JH, Guo C, Tang YL, Xiang JF, Chen S, Wang J, Liu HZ. Micellization in aqueous solution of an ethylene oxide-propylene oxide triblock copolymer, investigated with $1 \mathrm{H}$ NMR spectroscopy, pulsed-field gradient NMR, and NMR relaxation. J Colloid Interface Sci 2007;312:390-6.

[39] Zana R, Marques C, Johner A. Dynamics of micelles of the triblock copolymers 
poly(ethylene oxide)-poly(propylene oxide)-poly(ethylene oxide) in aqueous solution. Adv Colloid Interface Sci 2006;123:345-51.

[40] Pacovská M, Procházka K, Tuzar Z, Munk P. Formation of block copolymer micelles: a sedimentation study. Polymer 1993;34:4585-8.

[41] Alexandridis P, Alan Hatton T. Poly(ethylene oxide)-poly(propylene oxide)-poly(ethylene oxide) block copolymer surfactants in aqueous solutions and at interfaces:

thermodynamics, structure, dynamics, and modeling. Colloids Surf Physicochem Eng Aspects 1995;96:1-46.

[42] Kabanov AV, Nazarova IR, Astafieva IV, Batrakova EV, Alakhov VY, Yaroslavov AA, Kabanov VA. Micelle Formation and Solubilization of Fluorescent Probes in Poly(oxyethylene-b-oxypropylene-b-oxyethylene) Solutions. Macromolecules $1995 ; 28: 2303-14$.

[43] Gaucher G, Dufresne MH, Sant VP, Kang N, Maysinger D, Leroux JC. Block copolymer micelles: preparation, characterization and application in drug delivery. J Controlled Release 2005;109:169-88.

[44] Park JS, Akiyama Y, Yamasaki Y, Kataoka K. Preparation and characterization of polyion complex micelles with a novel thermosensitive poly(2-isopropyl-2-oxazoline) shell via the complexation of oppositely charged block ionomers. Langmuir 2007;23:138-46.

[45] Li MQ, Song WT, Tang ZH, Lv SX, Lin L, Sun H, Li QS, Yang Y, Hong H, Chen XS. Nanoscaled poly(L-glutamic acid)/doxorubicin-amphiphile complex as $\mathrm{pH}$-responsive drug delivery system for effective treatment of nonsmall cell lung cancer. ACS Appl Mater Interfaces 2013;5:1781-92.

[46] Nishiyama N, Okazaki S, Cabral H, Miyamoto M, Kato Y, Sugiyama Y, Nishio K, Matsumura Y, Kataoka K. Novel cisplatin-incorporated polymeric micelles can eradicate solid tumors in mice. Cancer Res 2003;63:8977-83. 
[47] Lee H, Zeng F, Dunne M, Allen C. Methoxy poly(ethylene

glycol)-block-poly $(\delta$-valerolactone $)$ copolymer micelles for formulation of hydrophobic drugs. Biomacromolecules 2005;6:3119-28.

[48] Kim SC, Kim DW, Shim YH, Bang JS, Oh HS, Kim SW, Seo MH. In vivo evaluation of polymeric micellar paclitaxel formulation: toxicity and efficacy. J Controlled Release 2001;72:191-202.

[49] Ke XY, Lin Ng VW, Gao SJ, Tong YW, Hedrick JL, Yang YY. Co-delivery of thioridazine and doxorubicin using polymeric micelles for targeting both cancer cells and cancer stem cells. Biomaterials 2014;35:1096-108.

[50] Yang H, Mao H, Wan Z, Zhu A, Guo M, Li Y, Li X, Wan J, Yang X, Shuai X, Chen H. Micelles assembled with carbocyanine dyes for theranostic near-infrared fluorescent cancer imaging and photothermal therapy. Biomaterials 2013;34:9124-33.

[51] La SB, Okano T, Kataoka K. Preparation and characterization of the micelle-forming polymeric drug indomethacin-incorporated poly(ethylene oxide)-poly( $\beta$-benzyl L-aspartate) block copolymer micelles. J Pharm Sci 1996;85:85-90.

[52] Huh KM, Lee SC, Cho YW, Lee J, Jeong JH, Park K. Hydrotropic polymer micelle system for delivery of paclitaxel. J Controlled Release 2005;101:59-68.

[53] Vangeyte P, Gautier S, Jérôme R. About the methods of preparation of poly(ethylene oxide)-b-poly( $\varepsilon$-caprolactone) nanoparticles in water: Analysis by dynamic light scattering. Colloids Surf Physicochem Eng Aspects 2004;242:203-11.

[54] Zhou Z, Ma X, Jin E, Tang J, Sui M, Shen Y, Van Kirk EA, Murdoch WJ, Radosz M. Linear-dendritic drug conjugates forming long-circulating nanorods for cancer-drug delivery. Biomaterials 2013;34:5722-35.

[55] Wang HX, Xiong MH, Wang YC, Zhu J, Wang J. N-acetylgalactosamine functionalized mixed micellar nanoparticles for targeted delivery of siRNA to liver. J Controlled Release 
2013;166:106-14.

[56] Sant VP, Smith D, Leroux JC. Enhancement of oral bioavailability of poorly water-soluble drugs by poly(ethylene glycol)-block-poly(alkyl acrylate-co-methacrylic acid)

self-assemblies. J Controlled Release 2005;104:289-300.

[57] Sant VP, Smith D, Leroux JC. Novel pH-sensitive supramolecular assemblies for oral delivery of poorly water soluble drugs: preparation and characterization. J Controlled Release 2004;97:301-12.

[58] Gong C, Shi S, Wang X, Wang Y, Fu S, Dong P, Chen L, Zhao X, Wei Y, Qian Z. Novel composite drug delivery system for honokiol delivery: self-assembled poly(ethylene glycol)-poly(epsilon-caprolactone)-poly(ethylene glycol) micelles in thermosensitive poly(ethylene glycol)-poly(e-caprolactone)-poly(ethylene glycol) hydrogel. The Journal of Physical Chemistry B 2009;113:10183-8.

[59] Lv SX, Li MQ, Tang ZH, Song WT, Sun H, Liu HY, Chen XS. Doxorubicin-loaded amphiphilic polypeptide-based nanoparticles as an efficient drug delivery system for cancer therapy. Acta Biomater 2013;9:9330-42.

[60] Cabral H, Nishiyama N, Okazaki S, Koyama H, Kataoka K. Preparation and biological properties of dichloro(1,2-diaminocyclohexane)platinum(II) (DACHPt)-loaded polymeric micelles. J Controlled Release 2005;101:223-32.

[61] Nishiyama N, Yokoyama M, Aoyagi T, Okano T, Sakurai Y, Kataoka K. Preparation and Characterization of Self-Assembled Polymer-Metal Complex Micelle from cis-Dichlorodiammineplatinum(II) and Poly(ethylene glycol)-Poly $(\alpha, \beta$-aspartic acid) Block Copolymer in an Aqueous Medium. Langmuir 1999;15:377-83.

[62] Huang Y, Tang ZH, Zhang XF, Yu HY, Sun H, Pang X, Chen XS. pH-Triggered Charge-Reversal Polypeptide Nanoparticles for Cisplatin Delivery: Preparation and In Vitro Evaluation. Biomacromolecules 2013;14:2023-32. 
[63] Song WT, Li MQ, Tang ZH, Li QS, Yang Y, Liu HY, Duan TC, Hong H, Chen XS. Methoxypoly(ethylene glycol)-block-poly(L-glutamic acid)-loaded cisplatin and a combination with iRGD for the treatment of non-small-cell lung cancers. Macromol Biosci 2012;12:1514-23.

[64] Yu H, Tang Z, Zhang D, Song W, Zhang Y, Yang Y, Ahmad Z, Chen X. Pharmacokinetics, biodistribution and in vivo efficacy of cisplatin loaded poly(L-glutamic acid)-g-methoxy poly(ethylene glycol) complex nanoparticles for tumor therapy. J Controlled Release 2015;205:89-97.

[65] Ahmad Z, Tang Z, Shah A, Lv S, Zhang D, Zhang Y, Chen X. Cisplatin Loaded Methoxy Poly (ethylene glycol)-block-Poly (L-glutamic acid-co-L-Phenylalanine) Nanoparticles against Human Breast Cancer Cell. Macromol Biosci 2014;14:1337-45.

[66] Li Y, Yu H, Sun H, Wang D, Yu L, Chen X. Cisplatin-loaded poly(L-glutamic acid)-g-methoxy poly(ethylene glycol) nanoparticlesas a potential chemotherapeutic agent against osteosarcoma. Chin J Polym Sci 2015;33:763-71.

[67] Song WT, Tang ZH, Zhang DW, Yu HY, Chen XS. Coadministration of vascular disrupting agents and nanomedicines to eradicate tumors from peripheral and central regions. Small 2015;11:3755-61.

[68] Shi C, Yu H, Sun D, Ma L, Tang Z, Xiao Q, Chen X. Cisplatin-loaded polymeric nanoparticles: Characterization and potential exploitation for the treatment of non-small cell lung carcinoma. Acta Biomater 2015;18:68-76.

[69] Riess G. Micellization of block copolymers. Prog Polym Sci 2003;28:1107-70.

[70] Jin Q, Liu XS, Liu GY, Ji J. Fabrication of core or shell reversibly photo cross-linked micelles and nanogels from double responsive water-soluble block copolymers. Polymer 2010;51:1311-9.

[71] Tyrrell ZL, Shen Y, Radosz M. Fabrication of micellar nanoparticles for drug delivery 
through the self-assembly of block copolymers. Prog Polym Sci 2010;35:1128-43.

[72] Zeng F, Liu J, Allen C. Synthesis and characterization of biodegradable poly(ethylene glycol)-block-poly(5-benzyloxy-trimethylene carbonate) copolymers for drug delivery. Biomacromolecules 2004;5:1810-7.

[73] Lin WJ, Juang LW, Lin CC. Stability and release performance of a series of pegylated copolymeric micelles. Pharm Res 2003;20:668-73.

[74] Diao YY, Li HY, Fu YH, Han M, Hu YL, Jiang HL, Tsutsumi Y, Wei QC, Chen DW, Gao JQ. Doxorubicin-loaded PEG-PCL copolymer micelles enhance cytotoxicity and intracellular accumulation of doxorubicin in adriamycin-resistant tumor cells. Int $\mathrm{J}$ Nanomedicine 2011;6:1955-62.

[75] Gref R, Minamitake Y, Peracchia MT, Trubetskoy V, Torchilin V, Langer R. Biodegradable long-circulating polymeric nanospheres. Science 1994;263:1600-3.

[76] Elhasi S, Astaneh R, Lavasanifar A. Solubilization of an amphiphilic drug by poly(ethylene oxide)-block-poly(ester) micelles. Eur J Pharm Biopharm 2007;65:406-13.

[77] Brinkhuis RP, Rutjes FPJT, van Hest JCM. Polymeric vesicles in biomedical applications. Polym Chem 2011;2:1449-62.

[78] Lee JS, Feijen J. Polymersomes for drug delivery: Design, formation and characterization. J Controlled Release 2012;161:473-83.

[79] LoPresti C, Lomas H, Massignani M, Smart T, Battaglia G. Polymersomes: nature inspired nanometer sized compartments. J Mater Chem 2009;19:3576-90.

[80] Liu GY, Chen CJ, Ji J. Biocompatible and biodegradable polymersomes as delivery vehicles in biomedical applications. Soft Matter 2012;8:8811-21.

[81] Massignani M, Lomas H, Battaglia G. Polymersomes: A Synthetic Biological Approach to Encapsulation and Delivery. In: Caruso F, editor. Modern Techniques for Nano- and Microreactors/-reactions: Springer Berlin Heidelberg; 2010. p. 115-54. 
[82] Erfani-Moghadam V, Nomani A, Zamani M, Yazdani Y, Najafi F, Sadeghizadeh M. A novel diblock of copolymer of (monomethoxy poly [ethylene glycol]-oleate) with a small hydrophobic fraction to make stable micelles/polymersomes for curcumin delivery to cancer cells. Int J Nanomedicine 2014;9:5541-54.

[83] Yassin MA, Appelhans D, Wiedemuth R, Formanek P, Boye S, Lederer A, Temme A, Voit B. Overcoming concealment effects of targeting moieties in the PEG corona: controlled permeable polymersomes decorated with folate-antennae for selective targeting of tumor cells. Small 2015;11:1580-91.

[84] Lai MH, Lee S, Smith CE, Kim K, Kong H. Tailoring Polymersome Bilayer Permeability Improves Enhanced Permeability and Retention Effect for Bioimaging. ACS Appl Mater Interfaces 2014;6:10821-9.

[85] Meng F, Zhong Z. Polymersomes Spanning from Nano- to Microscales: Advanced Vehicles for Controlled Drug Delivery and Robust Vesicles for Virus and Cell Mimicking. J Phys Chem Lett 2011;2:1533-9.

[86] Feng A, Yuan J. Smart nanocontainers: progress on novel stimuli-responsive polymer vesicles. Macromol Rapid Commun 2014;35:767-79.

[87] Wang X, Liu G, Hu J, Zhang G, Liu S. Concurrent block copolymer polymersome stabilization and bilayer permeabilization by stimuli-regulated "traceless" crosslinking. Angew Chem Int Ed 2014;126:3202-6.

[88] Zhang Y, Wu F, Yuan W, Jin T. Polymersomes of asymmetric bilayer membrane formed by phase-guided assembly. J Controlled Release 2010;147:413-9.

[89] Rodriguez-Garcia R, Mell M, Lopez-Montero I, Netzel J, Hellweg T, Monroy F. Polymersomes: smart vesicles of tunable rigidity and permeability. Soft Matter 2011;7:1532-42.

[90] Nicolas J, Mura S, Brambilla D, Mackiewicz N, Couvreur P. Design, functionalization 
strategies and biomedical applications of targeted biodegradable/biocompatible polymer-based nanocarriers for drug delivery. Chem Soc Rev 2013;42:1147-235.

[91] Chen YC, Chiang CF, Chen LF, Liang PC, Hsieh WY, Lin WL. Polymersomes conjugated with des-octanoyl ghrelin and folate as a BBB-penetrating cancer cell-targeting delivery system. Biomaterials 2014;35:4066-81.

[92] Israelachvili JN, Mitchell DJ, Ninham BW. Theory of self-assembly of hydrocarbon amphiphiles into micelles and bilayers. Journal of the Chemical Society, Faraday Transactions 2: Molecular and Chemical Physics 1976;72:1525-68.

[93] Yin H, Kang SW, Bae YH. Polymersome formation from AB2 Type 3-Miktoarm star copolymers. Macromolecules 2009;42:7456-64.

[94] Ayen WY, Garkhal K, Kumar N. Doxorubicin-Loaded (PEG)(3)-PLA Nanopolymersomes: Effect of Solvents and Process Parameters on Formulation Development and In Vitro Study. Mol Pharm 2011;8:466-78.

[95] Utada AS, Lorenceau E, Link DR, Kaplan PD, Stone HA, Weitz DA. Monodisperse double emulsions generated from a microcapillary device. Science 2005;308:537-41.

[96] Hayward RC, Utada AS, Dan N, Weitz DA. Dewetting instability during the formation of polymersomes from block-copolymer-stabilized double emulsions. Langmuir 2006;22:4457-61.

[97] Shum HC, Kim JW, Weitz DA. Microfluidic fabrication of monodisperse biocompatible and biodegradable polymersomes with controlled permeability. J Am Chem Soc 2008;130:9543-9.

[98] Shum HC, Zhao YJ, Kim SH, Weitz DA. Multicompartment Polymersomes from Double Emulsions. Angew Chem Int Ed 2011;50:1648-51.

[99] Hauschild S, Lipprandt U, Rumplecker A, Borchert U, Rank A, Schubert R, Förster S. Direct preparation and loading of lipid and polymer vesicles using inkjets. Small 
2005;1:1177-80.

[100] Maglio G, Nicodemi F, Conte C, Palumbo R, Tirino P, Panza E, Ianaro A, Ungaro F, Quaglia F. Nanocapsules based on linear and Y-shaped 3-miktoarm star-block PEO-PCL copolymers as sustained delivery system for hydrophilic molecules. Biomacromolecules 2011;12:4221-9.

[101] Holowka EP, Pochan DJ, Deming TJ. Charged Polypeptide Vesicles with Controllable Diameter. J Am Chem Soc 2005;127:12423-8.

[102] Malinova V, Belegrinou S, de Bruyn Ouboter D, Meier W. Biomimetic Block Copolymer Membranes. In: Grasser T, Meller G, Li L, editors. Org Electron: Springer Berlin Heidelberg; 2010. p. 213-58.

[103] Angelova MI, Dimitrov DS. Liposome electroformation. Faraday Discuss Chem Soc 1986;81:303-11.

[104] Hope MJ, Bally MB, Webb G, Cullis PR. Production of large unilamellar vesicles by a rapid extrusion procedure. Characterization of size distribution, trapped volume and ability to maintain a membrane potential. Biochim Biophys Acta - biomembranes $1985 ; 812: 55-65$.

[105] Napoli A, Valentini M, Tirelli N, Müller M, Hubbell JA. Oxidation-responsive polymeric vesicles. Nature Mater 2004;3:183-9.

[106] Howse JR, Jones RAL, Battaglia G, Ducker RE, Leggett GJ, Ryan AJ. Templated formation of giant polymer vesicles with controlled size distributions. Nature Mater 2009;8:507-11.

[107] O’Neil CP, Suzuki T, Demurtas D, Finka A, Hubbell JA. A novel method for the encapsulation of biomolecules into polymersomes via direct hydration. Langmuir 2009;25:9025-9.

[108] Xu H, Meng F, Zhong Z. Reversibly crosslinked temperature-responsive nano-sized 
polymersomes: synthesis and triggered drug release. J Mater Chem 2009;19:4183-90.

[109] Vauthier C, Bouchemal K. Methods for the preparation and manufacture of polymeric nanoparticles. Pharm Res 2009;26:1025-58.

[110] Sant S, Tao SL, Fisher OZ, Xu QB, Peppas NA, Khademhosseini A. Microfabrication technologies for oral drug delivery. Adv Drug Del Rev 2012;64:496-507.

[111] Acharya G, Shin CS, McDermott M, Mishra H, Park H, Kwon IC, Park K. The hydrogel template method for fabrication of homogeneous nano/microparticles. J Controlled Release 2010;141:314-9.

[112] van der Ende AE, Harrell J, Sathiyakumar V, Meschievitz M, Katz J, Adcock K, Harth E. “Click” Reactions: Novel Chemistries for Forming Well-defined Polyester Nanoparticles. Macromolecules 2010;43:5665-71.

[113] Harth E, Horn BV, Lee VY, Germack DS, Gonzales CP, Miller RD, Hawker CJ. A Facile Approach to Architecturally Defined Nanoparticles via Intramolecular Chain Collapse. J Am Chem Soc 2002;124:8653-60.

[114] Amato DV, Amato DN, Flynt AS, Patton DL. Functional, sub-100 nm polymer nanoparticles via thiol-ene miniemulsion photopolymerization. Polym Chem 2015;6:5625-32.

[115] Teekamp N, Duque LF, Frijlink HW, Hinrichs WL, Olinga P. Production methods and stabilization strategies for polymer-based nanoparticles and microparticles for parenteral delivery of peptides and proteins. Expert Opin Drug Deliv 2015;12:1311-31.

[116] Mabille C, Leal-Calderon L, Bibette J, Schmitt V. Monodisperse fragmentation in emulsions: Mechanisms and kinetics. Europhys Lett 2003;61:708-14.

[117] Umbanhowar PB, Prasad V, Weitz DA. Monodisperse emulsion generation via drop break off in a coflowing stream. Langmuir 2000;16:347-51.

[118] Sugiura S, Nakajima M, Iwamoto S, Seki M. Interfacial tension driven monodispersed 
droplet formation from microfabricated channel array. Langmuir 2001;17:5562-6.

[119] Kobayashi I, Mukataka S, Nakajima M. Effects of Type and Physical Properties of Oil Phase on Oil-in-Water Emulsion Droplet Formation in Straight-Through Microchannel Emulsification, Experimental and CFD Studies. Langmuir 2005;21:5722-30.

[120] Xu JH, Li SW, Tan J, Wang YJ, Luo GS. Controllable preparation of monodisperse O/W and W/O emulsions in the same microfluidic device. Langmuir 2006;22:7943-6.

[121] Charcosset C, Fessi H. Preparation of nanoparticles with a membrane contactor. J Membr Sci 2005;266:115-20.

[122] Joscelyne SM, Trägårdh G. Membrane emulsification - a literature review. J Membr Sci 2000;169:107-17.

[123] Wu J, Fan QZ, Xia YF, Ma GH. Uniform-sized particles in biomedical field prepared by membrane emulsification technique. Chem Eng Sci 2015;125:85-97.

[124] Quintanar-Guerrero D, Allemann E, Fessi H, Doelker E. Preparation techniques and mechanisms of formation of biodegradable nanoparticles from preformed polymers. Drug Dev Ind Pharm 1998;24:1113-28.

[125] Zhang S, Jiang Y, Chen CS, Spurgin J, Schwehr KA, Quigg A, Chin WC, Santschi PH. Aggregation, dissolution, and stability of quantum dots in marine environments: importance of extracellular polymeric substances. Environ Sci Technol 2012;46:8764-72.

[126] Herzi S, Essafi W, Bellagha S, Leal-Calderon F. Influence of the inner droplet fraction on the release rate profiles from multiple W/O/W emulsions. Colloids Surf Physicochem Eng Aspects 2014;441:489-95.

[127] Jeong YI, Na HS, Seo DH, Kim DG, Lee HC, Jang MK, Na SK, Roh SH, Kim SI, Nah JW. Ciprofloxacin-encapsulated poly(DL-lactide-co-glycolide) nanoparticles and its antibacterial activity. Int J Pharm 2008;352:317-23.

[128] Han Y, Tian H, He P, Chen X, Jing X. Insulin nanoparticle preparation and encapsulation 
into poly(lactic-co-glycolic acid) microspheres by using an anhydrous system. Int J Pharm 2009;378:159-66.

[129] Anton N, Benoit JP, Saulnier P. Design and production of nanoparticles formulated from nano-emulsion templates - A review. J Controlled Release 2008;128:185-99.

[130] Bouchemal K, Briancon S, Perrier E, Fessi H. Nano-emulsion formulation using spontaneous emulsification: solvent, oil and surfactant optimisation. Int J Pharm 2004;280:241-51.

[131] Weiss CK, Ziener U, Landfester K. A route to nonfunctionalized and functionalized poly(n-butylcyanoacrylate) nanoparticles: preparation in miniemulsion. Macromolecules 2007;40:928-38.

[132] Asua JM. Challenges for industrialization of miniemulsion polymerization. Prog Polym Sci 2014;39:1797-826.

[133] Desgouilles S, Vauthier C, Bazile D, Vacus J, Grossiord JL, Veillard M, Couvreur P. The Design of Nanoparticles Obtained by Solvent Evaporation: A Comprehensive Study. Langmuir 2003;19:9504-10.

[134] Quintanar-Guerrero D, Allemann E, Fessi H, Doelker E. Pseudolatex preparation using a novel emulsion-diffusion process involving direct displacement of partially water-miscible solvents by distillation. Int J Pharm 1999;188:155-64.

[135] Leroux JC, Allemann E, Doelker E, Gurny R. New approach for the preparation of nanoparticles by an emulsification-diffusion method. Eur J Pharm Biopharm $1995 ; 41: 14-8$.

[136] Ibrahim H, Bindschaedler C, Doelker E, Buri P, Gurny R. Aqueous nanodispersions prepared by a salting-out process. Int J Pharm 1992;87:239-46.

[137] Wang N, Wu XS. Preparation and characterization of agarose hydrogel nanoparticles for protein and peptide drug delivery. Pharm Dev Technol 1997;2:135-42. 
[138] Paques JP, Sagis LM, van Rijn CJ, van der Linden E. Nanospheres of alginate prepared through w/o emulsification and internal gelation with nanoparticles of $\mathrm{CaCO} 3$. Food Hydrocolloids 2014;40:182-8.

[139] Vauthier C, Labarre D, Ponchel G. Design aspects of poly(alkylcyanoacrylate) nanoparticles for drug delivery. J Drug Target 2007;15:641-63.

[140] Graf A, McDowell A, Rades T. Poly(alkycyanoacrylate) nanoparticles for enhanced delivery of therapeutics - is there real potential? Expert Opin Drug Deliv 2009;6:371-87.

[141] Kulkarni RK, Bartak DE, Leonard F. Initiation of polymerization of alkyl 2-cyanoacrylates in aqueous solutions of glycine and its derivatives. J Polym Sci A Polym Chem $1971 ; 9: 2977-81$.

[142] Nicolas J, Couvreur P. Synthesis of poly(alkyl cyanoacrylate)-based colloidal nanomedicines. Wiley Interdiscip Rev Nanomed Nanobiotechnol 2009;1:111-27.

[143] Hansali F, Wu M, Bendedouch D, Marie E. n-Butyl cyanoacrylate miniemulsion polymerization via the phase inversion composition method. Colloids Surf Physicochem Eng Aspects 2012;393:133-8.

[144] Chauvierre C, Labarre D, Couvreur P, Vauthier C. Radical emulsion polymerization of alkylcyanoacrylates initiated by the redox system dextran-cerium(IV) under acidic aqueous conditions. Macromolecules 2003;36:6018-27.

[145] Zandanel C, Vauthier C. Characterization of fluorescent poly (isobutylcyanoacrylate) nanoparticles obtained by copolymerization of a fluorescent probe during Redox Radical Emulsion Polymerization (RREP). Eur J Pharm Biopharm 2012;82:66-75.

[146] Alhareth K, Vauthier C, Gueutin C, Ponchel G, Moussa F. Doxorubicin loading and in vitro release from poly (alkylcyanoacrylate) nanoparticles produced by redox radical emulsion polymerization. J Appl Polym Sci 2011;119:816-22.

[147] Meier W. Polymer nanocapsules. Chem Soc Rev 2000;29:295-303. 
[148] Shutava TG, Pattekari PP, Arapov KA, Torchilin VP, Lvov YM. Architectural layer-by-layer assembly of drug nanocapsules with PEGylated polyelectrolytes. Soft matter 2012;8:9418-27.

[149] Ramasamy T, Haidar ZS, Tran TH, Choi JY, Jeong JH, Shin BS, Choi HG, Yong CS, Kim JO. Layer-by-layer assembly of liposomal nanoparticles with PEGylated polyelectrolytes enhances systemic delivery of multiple anticancer drugs. Acta Biomater 2014;10:5116-27.

[150] Stewart S, Liu GJ. Hollow nanospheres from polyisoprene-block-poly(2-cinnamoylethyl methacrylate)-block-poly(tert-butyl acrylate). Chem Mater 1999;11:1048-54.

[151] Dash BC, Rethore G, Monaghan M, Fitzgerald K, Gallagher W, Pandit A. The influence of size and charge of chitosan/polyglutamic acid hollow spheres on cellular internalization, viability and blood compatibility. Biomaterials 2010;31:8188-97.

[152] Itoh Y, Matsusaki M, Kida T, Akashi M. Locally controlled release of basic fibroblast growth factor from multilayered capsules. Biomacromolecules 2008;9:2202-6.

[153] Itoh Y, Matsusaki M, Kida T, Akashi M. Enzyme-responsive release of encapsulated proteins from biodegradable hollow capsules. Biomacromolecules 2006;7:2715-8.

[154] Kida T, Mouri M, Akashi M. Fabrication of hollow capsules composed of poly(methyl methacrylate) stereocomplex films. Angew Chem Int Ed 2006;45:7534-6.

[155] Mu B, Liu P, Pu QS. A functional cross linked polymeric nanocapsule with $\mathrm{pH}$-responsive brushes on its inner wall: Preparation, characterization and controlled release. React Funct Polym 2010;70:578-84.

[156] Mu B, Liu P, Tang ZB, Du PC, Dong Y. Temperature and pH dual-responsive cross-linked polymeric nanocapsules with controllable structures via surface-initiated atom transfer radical polymerization from templates. Nanomed-Nanotechnol 2011;7:789-96.

[157] Du PC, Mu B, Wang YJ, Shi HG, Xue DS, Liu P. Facile approach for temperature-responsive polymeric nanocapsules with movable magnetic cores. Mater Lett 
2011;65:1579-81.

[158] Huang X, Appelhans D, Formanek P, Simon F, Voit B. Synthesis of Well-Defined Photo-Cross-Linked Polymeric Nanocapsules by Surface-Initiated RAFT Polymerization. Macromolecules 2011;44:8351-60.

[159] Boyer C, Whittaker MR, Nouvel C, Davis TP. Synthesis of Hollow Polymer Nanocapsules Exploiting Gold Nanoparticles as Sacrificial Templates. Macromolecules 2010;43:1792-9.

[160] Sun L, Crooks RM, Chechik V. Preparation of polycyclodextrin hollow spheres by templating gold nanoparticles. Chem Commun 2001:359-60.

[161] Zhang K, Cutler JI, Zhang JA, Zheng D, Auyeung E, Mirkin CA. Nanopod Formation through Gold Nanoparticle Templated and Catalyzed Cross-linking of Polymers Bearing Pendant Propargyl Ethers. J Am Chem Soc 2010;132:15151-3.

[162] Liu WJ, He GH, Qian Z. Preparation of chitosan nanocapsules and their release properties. Polym Bull 2011;67:1237-44.

[163] Du PC, Liu P, Mu B, Wang YJ. Monodisperse Superparamagnetic pH-Sensitive Single-Layer Chitosan Hollow Microspheres with Controllable Structure. J Polym Sci A Polym Chem 2010;48:4981-8.

[164] Liu P, Liu GF, Zhang W, Jiang F. Crosslinked polymeric nanocapsules with controllable structure via a 'self-templating' approach. Nanotechnology 2010;21:015603/1-6.

[165] Dong Y, Mu B, Liu P. Temperature-responsive ionic-crosslinked polymeric nanocapsules via 'self-templating' approach. Colloids Surf B Biointerfaces 2011;84:267-71.

[166] Huang HY, Remsen EE, Kowalewski T, Wooley KL. Nanocages derived from shell cross-linked micelle templates. J Am Chem Soc 1999;121:3805-6.

[167] Cheng C, Qi K, Khoshdel E, Wooley KL. Tandem synthesis of core-shell brush copolymers and their transformation to peripherally cross-linked and hollowed nanostructures. J Am Chem Soc 2006;128:6808-9. 
[168] Zimmerman SC, Wendland MS, Rakow NA, Zharov I, Suslick KS. Synthetic hosts by monomolecular imprinting inside dendrimers. Nature 2002;418:399-403.

[169] Ding JX, Zhuang XL, Xiao CS, Cheng YL, Zhao L, He CL, Tang ZH, Chen XS.

Preparation of photo-cross-linked $\mathrm{pH}-$ responsive polypeptide nanogels as potential carriers for controlled drug delivery. J Mater Chem 2011;21:11383-91.

[170] Kabanov AV, Vinogradov SV. Nanogels as pharmaceutical carriers: finite networks of infinite capabilities. Angew Chem Int Ed 2009;48:5418-29.

[171] McAllister K, Sazani P, Adam M, Cho MJ, Rubinstein M, Samulski RJ, DeSimone JM. Polymeric nanogels produced via inverse microemulsion polymerization as potential gene and antisense delivery agents. J Am Chem Soc 2002;124:15198-207.

[172] Raemdonck K, Naeye B, Buyens K, Vandenbroucke RE, Hogset A, Demeester J, De Smedt SC. Biodegradable Dextran Nanogels for RNA Interference: Focusing on Endosomal Escape and Intracellular siRNA Delivery. Adv Funct Mater 2009;19:1406-15.

[173] Tamura M, Ichinohe S, Tamura A, Ikeda Y, Nagasaki Y. In vitro and in vivo characteristics of core-shell type nanogel particles: Optimization of core cross-linking density and surface poly(ethylene glycol) density in PEGylated nanogels. Acta Biomater 2011;7:3354-61.

[174] Nakamura T, Tamura A, Murotani H, Oishi M, Jinji Y, Matsuishi K, Nagasaki Y. Large payloads of gold nanoparticles into the polyamine network core of stimuli-responsive PEGylated nanogels for selective and noninvasive cancer photothermal therapy. Nanoscale 2010;2:739-46.

[175] Oishi M, Sumitani S, Bronich TK, Kabanov AV, Boska MD, Nagasaki Y. Novel F-19 MRS/I Nanoprobe Based on pH-Responsive PEGylated Nanogel: pH-Dependent F-19 Magnetic Resonance Studies. Chem Lett 2009;38:128-9.

[176] Oishi M, Tamura A, Nakamura T, Nagasaki Y. A Smart Nanoprobe Based On Fluorescence-Quenching PEGylated Nanogels Containing Gold Nanoparticles for 
Monitoring the Response to Cancer Therapy. Adv Funct Mater 2009;19:827-34.

[177] Khondee S, Yakovleva T, Berkland C. Low Charge Polyvinylamine Nanogels Offer Sustained, Low-Level Gene Expression. J Appl Polym Sci 2010;118:1921-32.

[178] Abu Samah N, Williams N, Heard CM. Nanogel particulates located within diffusion cell receptor phases following topical application demonstrates uptake into and migration across skin. Int J Pharm 2010;401:72-8.

[179] Ramos J, Imaz A, Forcada J. Temperature-sensitive nanogels: poly(N-vinylcaprolactam) versus poly (N-isopropylacrylamide). Polym Chem 2012;3:852-6.

[180] Zhao Y, Zheng C, Wang Q, Fang J, Zhou G, Zhao H, Yang Y, Xu H, Feng G, Yang X. Permanent and Peripheral Embolization: Temperature-Sensitive p(N-Isopropylacrylamide-co-butyl Methylacrylate) Nanogel as a Novel Blood-Vessel-Embolic Material in the Interventional Therapy of Liver Tumors. Adv Funct Mater 2011;21:2035-42.

[181] Huang X, Misra GP, Vaish A, Flanagan JM, Sutermaster B, Lowe TL. Novel Nanogels with Both Thermoresponsive and Hydrolytically Degradable Properties. Macromolecules 2008;41:8339-45.

[182] Li CH, Liu SY. Responsive nanogel-based dual fluorescent sensors for temperature and Hg2+ ions with enhanced detection sensitivity. J Mater Chem 2010;20:10716-23.

[183] Qiao ZY, Zhang R, Du FS, Liang DH, Li ZC. Multi-responsive nanogels containing motifs of ortho ester, oligo(ethylene glycol) and disulfide linkage as carriers of hydrophobic anti-cancer drugs. J Controlled Release 2011;152:57-66.

[184] Oh JK, Bencherif SA, Matyjaszewski K. Atom transfer radical polymerization in inverse miniemulsion: A versatile route toward preparation and functionalization of microgels/nanogels for targeted drug delivery applications. Polymer 2009;50:4407-23.

[185] Averick SE, Magenau AJD, Simakova A, Woodman BF, Seong A, Mehl RA, 
Matyjaszewski K. Covalently incorporated protein-nanogels using AGET ATRP in an inverse miniemulsion. Polym Chem 2011;2:1476-8.

[186] Oh JK, Tang CB, Gao HF, Tsarevsky NV, Matyjaszewski K. Inverse miniemulsion ATRP: a new method for synthesis and functionalization of well-defined water-soluble/cross-linked polymeric particles. J Am Chem Soc 2006;128:5578-84.

[187] Min K, Gao H, Yoon JA, Wu W, Kowalewski T, Matyjaszewski K. One-Pot Synthesis of Hairy Nanoparticles by Emulsion ATRP. Macromolecules 2009;42:1597-603.

[188] Oh JK, Siegwart DJ, Matyjaszewski K. Synthesis and biodegradation of nanogels as delivery carriers for carbohydrate drugs. Biomacromolecules 2007;8:3326-31.

[189] Oh JK, Siegwart DJ, Lee HI, Sherwood G, Peteanu L, Hollinger JO, Kataoka K, Matyjaszewski K. Biodegradable nanogels prepared by atom transfer radical polymerization as potential drug delivery carriers: synthesis, biodegradation, in vitro release, and bioconjugation. J Am Chem Soc 2007;129:5939-45.

[190] Pich A, Richtering W. Microgels by Precipitation Polymerization: Synthesis, Characterization, and Functionalization. In: Pich A, Richtering W, editors. Chemical Design of Responsive Microgels. Berlin, Heidelberg: Springer Berlin Heidelberg; 2011. p. $1-37$.

[191] Pelton RH, Chibante P. Preparation of Aqueous Lattices with N-Isopropylacrylamide. Colloid Surface 1986;20:247-56.

[192] Schachschal S, Balaceanu A, Melian C, Demco DE, Eckert T, Richtering W, Pich A. Polyampholyte Microgels with Anionic Core and Cationic Shell. Macromolecules 2010;43:4331-9.

[193] Blackburn WH, Dickerson EB, Smith MH, McDonald JF, Lyon LA. Peptide-Functionalized Nanogels for Targeted siRNA Delivery. Bioconjugate Chem 2009;20:960-8. 
[194] Wang J, Sutti A, Wang X, Lin T. Thermo-responsive

Hercosett/Poly(N-isopropylacrylamide) films: A new, fast, optically responsive coating. J Colloid Interface Sci 2012;369:231-7.

[195] Li X, Zuo J, Guo YL, Yuan XH. Preparation and characterization of narrowly distributed nanogels with temperature-responsive core and $\mathrm{pH}$-responsive shell. Macromolecules 2004;37:10042-6.

[196] Wu WT, Mitra N, Yan ECY, Zhou SQ. Multifunctional hybrid nanogel for integration of optical glucose sensing and self-regulated insulin release at physiological $\mathrm{pH}$. ACS Nano 2010;4:4831-9.

[197] Dickerson EB, Blackburn WH, Smith MH, Kapa LB, Lyon LA, McDonald JF. Chemosensitization of cancer cells by siRNA using targeted nanogel delivery. BMC Cancer 2010;10:1-11.

[198] Liang Y, Kiick KL. Multifunctional lipid-coated polymer nanogels crosslinked by photo-triggered Michael-type addition. Polym Chem 2014;5:1728-36.

[199] Wu WT, Aiello M, Zhou T, Berliner A, Banerjee P, Zhou SQ. In-situ immobilization of quantum dots in polysaccharide-based nanogels for integration of optical $\mathrm{pH}$-sensing, tumor cell imaging, and drug delivery. Biomaterials 2010;31:3023-31.

[200] Su SS, Wang H, Liu XG, Wu Y, Nie GJ. iRGD-coupled responsive fluorescent nanogel for targeted drug delivery. Biomaterials 2013;34:3523-33.

[201] Akiyoshi K, Kobayashi S, Shichibe S, Mix D, Baudys M, Kim SW, Sunamoto J. Self-assembled hydrogel nanoparticle of cholesterol-bearing pullulan as a carrier of protein drugs: Complexation and stabilization of insulin. J Controlled Release 1998;54:313-20.

[202] Kim JO, Sahay G, Kabanov AV, Bronich TK. Polymeric micelles with ionic cores containing biodegradable cross-links for delivery of chemotherapeutic agents. 
Biomacromolecules 2010;11:919-26.

[203] He J, Yan B, Tremblay L, Zhao Y. Both core- and shell-cross-linked nanogels:

photoinduced size change, intraparticle LCST, and interparticle UCST thermal behaviors. Langmuir 2011;27:436-44.

[204] Li YL, Zhu L, Liu Z, Cheng R, Meng F, Cui JH, Ji SJ, Zhong Z. Reversibly Stabilized Multifunctional Dextran Nanoparticles Efficiently Deliver Doxorubicin into the Nuclei of Cancer Cells. Angew Chem Int Ed 2009;48:9914-8.

[205] Ryu JH, Chacko RT, Jiwpanich S, Bickerton S, Babu RP, Thayumanavan S. Self-cross-linked polymer nanogels: a versatile nanoscopic drug delivery platform. J Am Chem Soc 2010;132:17227-35.

[206] Sanson N, Rieger J. Synthesis of nanogels/microgels by conventional and controlled radical crosslinking copolymerization. Polym Chem 2010;1:965-77.

[207] Gao H, Li W, Matyjaszewski K. Synthesis of polyacrylate networks by ATRP: Parameters influencing experimental gel points. Macromolecules 2008;41:2335-40.

[208] Gao H, Matyjaszewski K. Synthesis of functional polymers with controlled architecture by CRP of monomers in the presence of cross-linkers: From stars to gels. Prog Polym Sci 2009;34:317-50.

[209] Wiltshire JT, Qiao GG. Selectively degradable core cross-linked star polymers. Macromolecules 2006;39:9018-27.

[210] Xiong MH, Wu J, Wang YC, Li LS, Liu XB, Zhang GZ, Yan LF, Wang J. Synthesis of PEG-Armed and Polyphosphoester Core-Cross-Linked Nanogel by One-Step Ring-Opening Polymerization. Macromolecules 2009;42:893-6.

[211] Xiong MH, Li YJ, Bao Y, Yang XZ, Hu B, Wang J. Bacteria-responsive multifunctional nanogel for targeted antibiotic delivery. Adv Mater 2012;24:6175-80.

[212] Xing T, Lai B, Ye X, Yan L. Disulfide core cross-linked PEGylated polypeptide nanogel 
prepared by a one-step ring opening copolymerization of $\mathrm{N}$-carboxyanhydrides for drug delivery. Macromol Biosci 2011;11:962-9.

[213] Ding J, Shi F, Xiao C, Lin L, Chen L, He C, Zhuang X, Chen X. One-step preparation of reduction-responsive poly(ethylene glycol)-poly(amino acid)s nanogels as efficient intracellular drug delivery platforms. Polym Chem 2011;2:2857-64.

[214] Shi F, Ding J, Xiao C, Zhuang X, He C, Chen L, Chen X. Intracellular microenvironment responsive PEGylated polypeptide nanogels with ionizable cores for efficient doxorubicin loading and triggered release. J Mater Chem 2012;22:14168-79.

[215] Zhou FL, Gong RH. Manufacturing technologies of polymeric nanofibres and nanofibre yarns. Polym Int 2008;57:837-45.

[216] Nayak R, Padhye R, Kyratzis I, Truong YB, Arnold L. Recent advances in nanofibre fabrication techniques. Text Res J 2012;82:129-47.

[217] Burger C, Hsiao BS, Chu B. Nanofibrous materials and their applications. Ann Rev Mater Res 2006;36:333-68.

[218] Greiner A, Wendorff JH. Electrospinning: A fascinating method for the preparation of ultrathin fibres. Angew Chem Int Ed 2007;46:5670-703.

[219] Agarwal S, Wendorff JH, Greiner A. Use of electrospinning technique for biomedical applications. Polymer 2008;49:5603-21.

[220] Li D, Xia Y. Electrospinning of nanofibers: reinventing the wheel? Adv Mater 2004;16:1151-70.

[221] Jiang T, Carbone EJ, Lo KWH, Laurencin CT. Electrospinning of polymer nanofibers for tissue regeneration. Prog Polym Sci 2015;46:1-24.

[222] Tang Z, Qiu C, McCutcheon JR, Yoon K, Ma H, Fang D, Lee E, Kopp C, Hsiao BS, Chu B. Design and Fabrication of Electrospun Polyethersulfone Nanofibrous Scaffold for High-Flux Nanofiltration Membranes. J Polym Sci B Polym Phys 2009;47:2288-300. 
[223] Deitzel JM, Kleinmeyer J, Harris D, Tan NCB. The effect of processing variables on the morphology of electrospun nanofibers and textiles. Polymer 2001;42:261-72.

[224] Theron SA, Zussman E, Yarin AL. Experimental investigation of the governing parameters in the electrospinning of polymer solutions. Polymer 2004;45:2017-30.

[225] Tan SH, Inai R, Kotaki M, Ramakrishna S. Systematic parameter study for ultra-fine fiber fabrication via electrospinning process. Polymer 2005;46:6128-34.

[226] Larrondo L, St. John Manley R. Electrostatic fiber spinning from polymer melts. I. Experimental observations on fiber formation and properties. J Polymer Sci Polymer Phys Ed 1981;19:909-20.

[227] Li X, Zhang Y, Li H, Chen H, Ding Y, Yang W. Effect of oriented fiber membrane fabricated via needleless melt electrospinning on water filtration efficiency. Desalination 2014;344:266-73.

[228] Muerza-Cascante ML, Haylock D, Hutmacher DW, Dalton PD. Melt electrospinning and its technologization in tissue engineering. Tissue engineering Part B, Reviews 2015;21:187-202.

[229] Zhou HJ, Green TB, Joo YL. The thermal effects on electrospinning of polylactic acid melts. Polymer 2006;47:7497-505.

[230] Dalton PD, Grafahrend D, Klinkhammer K, Klee D, Moller M. Electrospinning of polymer melts: Phenomenological observations. Polymer 2007;48:6823-33.

[231] Ogata N, Yamaguchi S, Shimada N, Lu G, Iwata T, Nakane K, Ogihara T. Poly(lactide) nanofibers produced by a melt-electrospinning system with a laser melting device. J Appl Polym Sci 2007;104:1640-5.

[232] Zhou FL, Gong RH, Porat I. Mass production of nanofibre assemblies by electrostatic spinning. Polym Int 2009;58:331-42.

[233] Theron SA, Yarin AL, Zussman E, Kroll E. Multiple jets in electrospinning: experiment 
and modeling. Polymer 2005;46:2889-99.

[234] Yarin AL, Zussman E. Upward needleless electrospinning of multiple nanofibers. Polymer 2004;45:2977-80.

[235] Higham AK, Tang C, Landry AM, Pridgeon MC, Lee EM, Andrady AL, Khan SA. Foam electrospinning: A multiple jet, needle - less process for nanofiber production. AlChE J 2014;60:1355-64.

[236] Li D, Wu T, He N, Wang J, Chen W, He L, Huang C, EI-Hamshary HA, Al-Deyab SS, Ke Q. Three-dimensional polycaprolactone scaffold via needleless electrospinning promotes cell proliferation and infiltration. Colloids Surf B Biointerfaces 2014;121:432-43.

[237] Holopainen J, Penttinen T, Santala E, Ritala M. Needleless electrospinning with twisted wire spinneret. Nanotechnology 2015;26:025301/1-8.

[238] Xu XL, Zhuang XL, Chen XS, Wang XR, Yang LX, Jing XB. Preparation of core-sheath composite nanofibers by emulsion electrospinning. Macromol Rapid Commun 2006;27:1637-42.

[239] Xu XL, Yang LX, Xu XY, Wang X, Chen XS, Liang QZ, Zeng J, Jing XB. Ultrafine medicated fibers electrospun from W/O emulsions. J Controlled Release 2005;108:33-42.

[240] Sun ZC, Zussman E, Yarin AL, Wendorff JH, Greiner A. Compound core-shell polymer nanofibers by co-electrospinning. Adv Mater 2003;15:1929-32.

[241] Zhang YZ, Wang X, Feng Y, Li J, Lim CT, Ramakrishna S. Coaxial electrospinning of (fluorescein isothiocyanate-conjugated bovine serum albumin)-encapsulated poly(epsilon-caprolactone) nanofibers for sustained release. Biomacromolecules 2006;7:1049-57.

[242] Jiang H, Wang L, Zhu K. Coaxial electrospinning for encapsulation and controlled release of fragile water-soluble bioactive agents. J Controlled Release 2014;193:296-303.

[243] Yu H, Jia Y, Yao C, Lu Y. PCL/PEG core/sheath fibers with controlled drug release rate 
fabricated on the basis of a novel combined technique. Int J Pharm 2014;469:17-22.

[244] Jayaraman K, Kotaki M, Zhang YZ, Mo XM, Ramakrishna S. Recent advances in polymer nanofibers. J Nanosci Nanotechno 2004;4:52-65.

[245] Nam YS, Park TG. Biodegradable polymeric microcellular foams by modified thermally induced phase separation method. Biomaterials 1999;20:1783-90.

[246] Rowlands AS, Lim SA, Martin D, Cooper-White JJ. Polyurethane/poly(lactic-co-glycolic) acid composite scaffolds fabricated by thermally induced phase separation. Biomaterials 2007;28:2109-21.

[247] Li L, Ge J, Wang L, Guo B, Ma PX. Electroactive nanofibrous biomimetic scaffolds by thermally induced phase separation. J Mater Chem B Mater Biol Med 2014;2:6119-30.

[248] Beachley V, Wen X. Polymer nanofibrous structures: Fabrication, biofunctionalization, and cell interactions. Prog Polym Sci 2010;35:868-92.

[249] Siegel RW. Nanostructure science and technology: A global view. Abstr Pap Am Chem Soc 1998;216:U302-U302.

[250] Tao SL, Desai TA. Aligned arrays of biodegradable poly(epsilon-caprolactone) nanowires and nanofibers by template synthesis. Nano Lett 2007;7:1463-8.

[251] Chen Y, Zhang LN, Lu XY, Zhao N, Xu J. Morphology and crystalline structure of poly(epsilon-caprolactone) nanofiber via porous aluminium oxide template. Macromol Mater Eng 2006;291:1098-103.

[252] Li A, Hokugo A, Yalom A, Berns EJ, Stephanopoulos N, McClendon MT, Segovia LA, Spigelman I, Stupp SI, Jarrahy R. A bioengineered peripheral nerve construct using aligned peptide amphiphile nanofibers. Biomaterials 2014;35:8780-90.

[253] Cui H, Webber MJ, Stupp SI. Self-assembly of peptide amphiphiles: From molecules to nanostructures to biomaterials. Biopolymers 2010;94:1-18.

[254] Zhao XB, Pan F, Xu H, Yaseen M, Shan HH, Hauser CAE, Zhang SG, Lu JR. Molecular 
self-assembly and applications of designer peptide amphiphiles. Chem Soc Rev 2010;39:3480-98.

[255] Luo J, Tong YW. Self-assembly of collagen-mimetic peptide amphiphiles into biofunctional nanofiber. ACS Nano 2011;5:7739-47.

[256] Cooper A, Zhong C, Kinoshita Y, Morrison RS, Rolandi M, Zhang MQ. Self-assembled chitin nanofiber templates for artificial neural networks. J Mater Chem 2012;22:3105-9. O'Leary LER, Fallas JA, Bakota EL, Kang MK, Hartgerink JD. Multi-hierarchical self-assembly of a collagen mimetic peptide from triple helix to nanofibre and hydrogel. Nat Chem 2011;3:821-8.

[258] Kapllani A, Dillard C, Washington KE, Biewer MC, Stefan MC, Kalra V. Self-Assembly of Poly(3-hexylthiophene)-block-poly( $\gamma$-benzyl-L-glutamate) within Solution-Cast Films and Nanofibers. Macromol Mater Eng 2014;299:1484-93.

[259] Samad A, Alam MI, Saxena K. Dendrimers: a class of polymers in the nanotechnology for the delivery of active pharmaceuticals. Curr Pharm Des 2009;15:2958-69.

[260] Kesharwani P, Jain K, Jain NK. Dendrimer as nanocarrier for drug delivery. Prog Polym Sci 2014;39:268-307.

[261] Qiao Z, Shi XY. Dendrimer-based molecular imaging contrast agents. Prog Polym Sci $2015 ; 44: 1-27$.

[262] Sheiko SS, Sumerlin BS, Matyjaszewski K. Cylindrical molecular brushes: Synthesis, characterization, and properties. Prog Polym Sci 2008;33:759-85.

[263] Fréchet JMJ. Dendrimers and supramolecular chemistry. Proc Natl Acad Sci USA 2002;99:4782-7.

[264] Xu Z, Kahr M, Walker KL, Wilkins CL, Moore JS. Phenylacetylene Dendrimers by the Divergent, Convergent, and Double-Stage Convergent Methods. J Am Chem Soc 1994;116:4537-50. 
[265] Li A, Ma J, Sun G, Li Z, Cho S, Clark C, Wooley KL. One-pot, facile synthesis of well-defined molecular brush copolymers by a tandem RAFT and ROMP, “Grafting-through” strategy. J Polym Sci A Polym Chem 2012;50:1681-8.

[266] Li Z, Ma J, Lee NS, Wooley KL. Dynamic Cylindrical Assembly of Triblock Copolymers by a Hierarchical Process of Covalent and Supramolecular Interactions. J Am Chem Soc 2011;133:1228-31.

[267] Gao H, Matyjaszewski K. Synthesis of molecular brushes by "grafting onto" method: combination of ATRP and click reactions. J Am Chem Soc 2007;129:6633-9.

[268] Beers KL, Gaynor SG, Matyjaszewski K, Sheiko SS, Möller M. The synthesis of densely grafted copolymers by atom transfer radical polymerization. Macromolecules 1998;31:9413-5.

[269] Ishizu K, Satoh J. Synthesis of isopropenylbenzyl - terminated macromonomers and preparation of polymer brushes by anionic homopolymerization. J Appl Polym Sci 2003;87:1790-3.

[270] Yu Y, Chen CK, Law WC, Mok J, Zou J, Prasad PN, Cheng C. Well-defined degradable brush polymer-drug conjugates for sustained delivery of Paclitaxel. Mol Pharm 2013;10:867-74.

[271] Hörtz C, Birke A, Kaps L, Decker S, Wächtersbach E, Fischer K, Schuppan D, Barz M, Schmidt M. Cylindrical Brush Polymers with Polysarcosine Side Chains: A Novel Biocompatible Carrier for Biomedical Applications. Macromolecules 2015;48:2074-86.

[272] Johnson JA, Lu YY, Burts AO, Lim YH, Finn MG, Koberstein JT, Turro NJ, Tirrell DA, Grubbs RH. Core-Clickable PEG-Branch-Azide Bivalent-Bottle-Brush Polymers by ROMP: Grafting-Through and Clicking-To. J Am Chem Soc 2011;133:559-66.

[273] Zou J, Jafr G, Themistou E, Yap Y, Wintrob ZA, Alexandridis P, Ceacareanu AC, Cheng C. $\mathrm{pH}-$ Sensitive brush polymer-drug conjugates by ring-opening metathesis copolymerization. 
Chem Commun 2011;47:4493-5.

[274] Liao L, Liu J, Dreaden EC, Morton SW, Shopsowitz KE, Hammond PT, Johnson JA. A Convergent Synthetic Platform for Single-Nanoparticle Combination Cancer Therapy: Ratiometric Loading and Controlled Release of Cisplatin, Doxorubicin, and Camptothecin. J Am Chem Soc 2014;136:5896-9.

[275] Sowers MA, McCombs JR, Wang Y, Paletta JT, Morton SW, Dreaden EC, Boska MD,

Ottaviani MF, Hammond PT, Rajca A, Johnson JA. Redox-responsive branched-bottlebrush polymers for in vivo MRI and fluorescence imaging. Nat Commun 2014;5:5460/1-9.

[276] Du JZ, Tang LY, Song WJ, Shi Y, Wang J. Evaluation of Polymeric Micelles from Brush Polymer with Poly(e-caprolactone)-b-Poly(ethylene glycol) Side Chains as Drug Carrier. Biomacromolecules 2009;10:2169-74.

[277] Shi J, Schellinger JG, Johnson RN, Choi JL, Chou B, Anghel EL, Pun SH. Influence of histidine incorporation on buffer capacity and gene transfection efficiency of HPMA-co-oligolysine brush polymers. Biomacromolecules 2013;14:1961-70.

[278] Zhao P, Liu L, Feng X, Wang C, Shuai X, Chen Y. Molecular nanoworm with PCL core and PEO shell as a non - spherical carrier for drug delivery. Macromol Rapid Commun $2012 ; 33: 1351-5$

[279] Lee H-i, Pietrasik J, Sheiko SS, Matyjaszewski K. Stimuli-responsive molecular brushes. Prog Polym Sci 2010;35:24-44.

[280] Gaharwar AK, Peppas NA, Khademhosseini A. Nanocomposite hydrogels for biomedical applications. Biotechnol Bioeng 2014;111:441-53.

[281] Paul DR, Robeson LM. Polymer nanotechnology: Nanocomposites. Polymer 2008;49:3187-204.

[282] Ojijo V, Sinha Ray S. Processing strategies in bionanocomposites. Prog Polym Sci 
2013;38:1543-89.

[283] Bahrami M, Ranjbarian S. Production of micro- and nano-composite particles by supercritical carbon dioxide. J Supercrit Fluids 2007;40:263-83.

[284] Chronakis IS. Novel nanocomposites and nanoceramics based on polymer nanofibers using electrospinning process - A review. J Mater Process Technol 2005;167:283-93.

[285] Ringsdorf H. Structure and properties of pharmacologically active polymers. J Polym Sci Polym Symp 1975;51:135-53.

[286] Larson N, Ghandehari H. Polymeric Conjugates for Drug Delivery. Chem Mater 2012;24:840-53.

[287] Rademaker-Lakhai JM, Terret C, Howell SB, Baud CM, de Boer RF, Pluim D, Beijnen JH, Schellens JHM, Droz JP. A phase I and pharmacological study of the platinum polymer AP5280 given as an intravenous infusion once every 3 weeks in patients with solid tumors. Clin Cancer Res 2004;10:3386-95.

[288] Sarapa N, Britto MR, Speed W, Jannuzzo M, Breda M, James CA, Porro M, Rocchetti M, Wanders A, Mahteme H, Nygren P. Assessment of normal and tumor tissue uptake of MAG-CPT, a polymer-bound prodrug of camptothecin, in patients undergoing elective surgery for colorectal carcinoma. Cancer Chemother Pharmacol 2003;52:424-30.

[289] Wachters FM, Groen HJM, Maring JG, Gietema JA, Porro M, Dumez H, de Vries EGE, van Oosterom AT. A phase I study with MAG-camptothecin intravenously administered weekly for 3 weeks in a 4-week cycle in adult patients with solid tumours. Br J Cancer 2004;90:2261-7.

[290] Nowotnik DP. AP5346 (ProLindac ${ }^{\mathrm{TM}}$ ), A DACH platinum polymer conjugate in phase II trials against ovarian cancer. Curr Bioact Compd 2011;7:21-6.

[291] Seymour LW, Ferry DR, Kerr DJ, Rea D, Whitlock M, Poyner R, Boivin C, Hesslewood S, Twelves C, Blackie R. Phase II studies of polymer-doxorubicin (PK1, FCE28068) in the 
treatment of breast, lung and colorectal cancer. Int J Oncol 2009;34:1629-36.

[292] Seymour LW, Ferry DR, Anderson D, Hesslewood S, Julyan PJ, Poyner R, Doran J, Young AM, Burtles S, Kerr DJ, Cancer Research Campaign Phase IIICTc. Hepatic drug targeting: Phase I evaluation of polymer-bound doxorubicin. J Clin Oncol 2002;20:1668-76.

[293] Terwogt JMM, Huinink WWT, Schellens JHM, Schot M, Mandjes IAM, Zurlo MG, Rocchetti M, Rosing H, Koopman FJ, Beijnen JH. Phase I clinical and pharmacokinetic study of PNU166945, a novel water-soluble polymer-conjugated prodrug of paclitaxel. Anticancer Drugs 2001;12:315-23.

[294] Rowinsky EK, Rizzo J, Ochoa L, Takimoto CH, Forouzesh B, Schwartz G, Hammond LA, Patnaik A, Kwiatek J, Goetz A, Denis L, McGuire J, Tolcher AW. A phase I and pharmacokinetic study of pegylated camptothecin as a 1-hour infusion every 3 weeks in patients with advanced solid malignancies. J Clin Oncol 2003;21:148-57.

[295] Wolff R, Routt S, Riggs-Sauthier J, Zhang W, Persson H, Johnson RK. NKTR-105, a novel PEGylated-docetaxel, demonstrates superior anti-tumor activity compared to docetaxel in human non-small cell lung and colon cancer xenografts. Ejc Supplements 2008;6:141-141.

[296] Awada A, Leung ACF, Zhao C, Hannah A, Perez E. OT3-01-07: The BEACON Study (BrEAst Cancer Outcomes with NKTR-102): A Phase 3 Open-Label, Randomized, Multicenter Study of NKTR-102 Versus Treatment of Physician's Choice (TPC) in Patients (pts) with Locally Recurrent or Metastatic Breast Cancer (MBC) Previously Treated with an Anthracycline, a Taxane, and Capecitabine (ATC). Cancer Res 2011;71(Suppl 24): Abstr OT3-01-7.

[297] Al-Huniti N, Wang Y, Birmingham B. Population Pharmacokinetic (PPK) Modeling of NKTR-118 Using Phase 1 and Phase 2 Data. J Clin Pharmacol 2011;51:1329-1329.

[298] Kurzrock R, Goel S, Wheler J, Hong D, Fu SQ, Rezai K, Morgan-Linnell SK, Urien S, 
Mani S, Chaudhary I, Ghalib MH, Buchbinder A, Lokiec F, Mulcahy M. Safety, pharmacokinetics, and activity of EZN-2208, a novel conjugate of polyethylene glycol and SN38, in patients with advanced malignancies. Cancer 2012;118:6144-51.

[299] Homsi J, Simon GR, Garrett CR, Springett G, De Conti R, Chiappori AA, Munster PN, Burton MK, Stromatt S, Allievi C, Angiuli P, Eisenfeld A, Sullivan DM, Daud AI. Phase I trial of poly-L-glutamate camptothecin (CT-2106) administered weekly in patients with advanced solid malignancies. Clin Cancer Res 2007;13:5855-61.

[300] Langer CJ, O’Byrne KJ, Socinski MA, Mikhailov SM, Lesniewski-Kmak K, Smakal M, Ciuleanu TE, Orlov SV, Dediu M, Heigener D. Phase III trial comparing paclitaxel poliglumex (CT-2103, PPX) in combination with carboplatin versus standard paclitaxel and carboplatin in the treatment of PS 2 patients with chemotherapy-naive advanced non-small cell lung cancer. J Thorac Oncol 2008;3:623-30.

[301] O’Brien ME, Socinski MA, Popovich AY, Bondarenko IN, Tomova A, Bilynsky BT, Hotko YS, Ganul VL, Kostinsky IY, Eisenfeld AJ. Randomized phase III trial comparing single-agent paclitaxel Poliglumex (CT-2103, PPX) with single-agent gemcitabine or vinorelbine for the treatment of PS 2 patients with chemotherapy-naive advanced non-small cell lung cancer. J Thorac Oncol 2008;3:728-34.

[302] Soepenberg O, de Jonge MJ, Sparreboom A, de Bruin P, Eskens FA, de Heus G, Wanders J, Cheverton P, Ducharme MP, Verweij J. Phase I and pharmacokinetic study of DE-310 in patients with advanced solid tumors. Clin Cancer Res 2005;11:703-11.

[303] Danhauserriedl S, Hausmann E, Schick HD, Bender R, Dietzfelbinger H, Rastetter J, Hanauske AR. Phase I clinical and pharmacokinetic trial of dextran conjugated doxorubicin (AD-70, DOX-OXD). Invest New Drugs 1993;11:187-95.

[304] Fram RJ, Garbo LE, Weiss GJ, Shkolny D, Yurkovetskiy AV, Bethune C, Ramanathan RK, Edelman MJ, Sausville EA. Phase 1 study of XMT-1001, a novel water soluble 
camptothecin conjugate, given as an IV infusion every 3 weeks to patients with advanced solid tumors. Ejc Supplements 2010;8:180-180.

[305] Walsh MD, Hanna SK, Sen J, Rawal S, Cabral CB, Yurkovetskiy AV, Fram RJ, Lowinger TB, Zamboni WC. Pharmacokinetics and Antitumor Efficacy of XMT-1001, a Novel, Polymeric Topoisomerase I Inhibitor, in Mice Bearing HT-29 Human Colon Carcinoma Xenografts. Clin Cancer Res 2012;18:2591-602.

[306] Hamaguchi T, Doi T, Eguchi-Nakajima T, Kato K, Yamada Y, Shimada Y, Fuse N, Ohtsu A, Matsumoto S, Takanashi M, Matsumura Y. Phase I Study of NK012, a Novel SN-38-Incorporating Micellar Nanoparticle, in Adult Patients with Solid Tumors. Clin Cancer Res 2010;16:5058-66.

[307] Raefsky E, Spigel DR, Infante JR, Bendell JC, Jones SF, Lipman AJ, Trent D, Kawamura S, Greco FA, Hainsworth JD, Burris HA. Phase II study of NK012 in relapsed small cell lung cancer. J Clin Oncol (Meeting Abstracts) 2011;29:Abstr 7079.

[308] Matsumura Y, Hamaguchi T, Ura T, Muro K, Yamada Y, Shimada Y, Shirao K, Okusaka T, Ueno H, Ikeda M. Phase I clinical trial and pharmacokinetic evaluation of NK911, a micelle-encapsulated doxorubicin. Br J Cancer 2004;91:1775-81.

[309] Nakanishi T, Fukushima S, Okamoto K, Suzuki M, Matsumura Y, Yokoyama M, Okano T, Sakurai Y, Kataoka K. Development of the polymer micelle carrier system for doxorubicin. J Controlled Release 2001;74:295-302.

[310] Goodarzi N, Varshochian R, Kamalinia G, Atyabi F, Dinarvand R. A review of polysaccharide cytotoxic drug conjugates for cancer therapy. Carbohydr Polym 2013;92:1280-93.

[311] Mehvar R. Recent trends in the use of polysaccharides for improved delivery of therapeutic agents: Pharmacokinetic and pharmacodynamic perspectives. Curr Pharm Biotechnol 2003;4:283-302. 
[312] Liu Z, Wang Y, Zhang N. Micelle-like nanoassemblies based on polymer-drug conjugates as an emerging platform for drug delivery. Expert Opin Drug Deliv 2012;9:805-22.

[313] Oh EJ, Park K, Kim KS, Kim J, Yang JA, Kong JH, Lee MY, Hoffman AS, Hahn SK. Target specific and long-acting delivery of protein, peptide, and nucleotide therapeutics using hyaluronic acid derivatives. J Controlled Release 2010;141:2-12.

[314] Auzenne E, Ghosh SC, Khodadadian M, Rivera B, Farquhar D, Price RE, Ravoori M, Kundra V, Freedman RS, Klostergaard J. Hyaluronic acid-paclitaxel: Antitumor efficacy against CD44(+) human ovarian carcinoma xenografts. Neoplasia 2007;9:479-86.

[315] Laurent TC. Biochemistry of hyaluronan. Acta Otolaryngol Suppl 1987;442:7-24.

[316] Lee H, Lee K, Park TG. Hyaluronic acid-paclitaxel conjugate micelles: Synthesis, characterization, and antitumor activity. Bioconjugate Chem 2008;19:1319-25.

[317] Yang B, Zhang L, Turley EA. Identification of two hyaluronan-binding domains in the hyaluronan receptor RHAMM. J Biol Chem 1993;268:8617-23.

[318] Okuno S, Harada M, Yano T, Yano S, Kiuchi S, Tsuda N, Sakamura Y, Imai J, Kawaguchi T, Tsujihara K. Complete regression of xenografted human carcinomas by camptothecin analogue-carboxymethyl dextran conjugate (T-0128). Cancer Res 2000;60:2988-95.

[319] Sugahara SI, Kajiki M, Kuriyama H, Kobayashi TR. Complete regression of xenografted human carcinomas by a paclitaxel-carboxymethyl dextran conjugate (AZ10992). J Controlled Release 2007;117:40-50.

[320] Ueda Y, Munechika K, Kikukawa A, Kanoh Y, Yamanouchi K, Yokoyama K. Comparison of efficacy, toxicity and pharmacokinetics of free adriamycin and adriamycin linked to oxidized dextran in rats. Chem Pharm Bull (Tokyo) 1989;37:1639-41.

[321] Shih LB, Goldenberg DM, Xuan H, Lu H, Sharkey RM, Hall TC. Anthracycline immunoconjugates prepared by a site-specific linkage via an amino-dextran intermediate carrier. Cancer Res 1991;51:4192-8. 
[322] Prashanth KVH, Tharanathan RN. Depolymerized products of chitosan as potent inhibitors of tumor-induced angiogenesis. Biochim Biophys Acta - General Subjects $2005 ; 1722: 22-9$.

[323] Duncan R, Kopeček J, Rejmanová P, Lloyd JB. Targeting of $\mathrm{N}$-(2-hydroxypropyl)methacrylamide copolymers to liver by incorporation of galactose residues. Biochim Biophys Acta - General Subjects 1983;755:518-21.

[324] Vasey PA, Kaye SB, Morrison R, Twelves C, Wilson P, Duncan R, Thomson AH, Murray LS, Hilditch TE, Murray T, Burtles S, Fraier D, Frigerio E, Cassidy J, Canc Res Campaign Phase IIIC. Phase I clinical and pharmacokinetic study of PK1

[N-(2-hydroxypropyl)methacrylamide copolymer doxorubicin]: first member of a new class of chemotherapeutic agents-drug-polymer conjugates. Clin Cancer Res 1999;5:83-94.

[325] Pasut G, Veronese FM. PEG conjugates in clinical development or use as anticancer agents: An overview. Adv Drug Del Rev 2009;61:1177-88.

[326] Ding J, He C, Xiao C, Chen J, Zhuang X, Chen X. pH-responsive drug delivery systems based on clickable poly(L-glutamic acid)-grafted comb copolymers. Macromol Res 2012;20:292-301.

[327] Cheng YL, He CL, Xiao CS, Ding JX, Zhuang XL, Chen XS. Versatile synthesis of temperature-sensitive polypeptides by click grafting of oligo(ethylene glycol). Polym Chem 2011;2:2627-34.

[328] Ding J, Xiao C, Zhuang X, He C, Chen X. Direct formation of cationic polypeptide vesicle as potential carrier for drug and gene. Mater Lett 2012;73:17-20.

[329] Deming TJ. Synthetic polypeptides for biomedical applications. Prog Polym Sci 2007;32:858-75.

[330] Tomalia DA, Baker H, Dewald J, Hall M, Kallos G, Martin S, Roeck J, Ryder J, Smith P. 
Dendritic macromolecules: synthesis of starburst dendrimers. Macromolecules $1986 ; 19: 2466-8$.

Tomalia DA, Baker H, Dewald J, Hall M, Kallos G, Martin S, Roeck J, Ryder J, Smith P. A new class of polymers: starburst-dendritic macromolecules. Polym J 1985;17:117-32.

Hu X, Jing X. Biodegradable amphiphilic polymer-drug conjugate micelles. Expert Opin Drug Deliv 2009;6:1079-90.

Lv S, Tang Z, Zhang D, Song W, Li M, Lin J, Liu H, Chen X. Well-defined polymer-drug conjugate engineered with redox and $\mathrm{pH}$-sensitive release mechanism for efficient delivery of paclitaxel. J Controlled Release 2014;194:220-7.

Li Q, Lv S, Tang Z, Liu M, Zhang D, Yang Y, Chen X. A co-delivery system based on paclitaxel grafted mPEG-b-PLG loaded with doxorubicin: Preparation, in vitro and in vivo evaluation. Int J Pharm 2014;471:412-20.

Zhang X, Li Y, Chen X, Wang X, Xu X, Liang Q, Hu J, Jing X. Synthesis and characterization of the paclitaxel/MPEG-PLA block copolymer conjugate. Biomaterials 2005;26:2121-8.

[336] Xie Z, Lu T, Chen X, Lu C, Zheng Y, Jing X. Triblock poly(lactic acid)-b-poly(ethylene glycol)-b-poly(lactic acid)/paclitaxel conjugates: Synthesis, micellization, and cytotoxicity. J Appl Polym Sci 2007;105:2271-9.

[337] Yoo HS, Park TG. Biodegradable polymeric micelles composed of doxorubicin conjugated PLGA-PEG block copolymer. J Controlled Release 2001;70:63-70.

[338] Oledzka E, Horeglad P, Gruszczyńska Z, Plichta A, Nałęcz-Jawecki G, Sobczak M. Polylactide conjugates of camptothecin with different drug release abilities. Molecules 2014;19:19460-70.

[339] Yu Y, Chen CK, Law WC, Weinheimer E, Sengupta S, Prasad PN, Cheng C. Polylactide-graft-doxorubicin nanoparticles with precisely controlled drug loading for 
pH-triggered drug delivery. Biomacromolecules 2014;15:524-32.

[340] Tong R, Cheng J. Paclitaxel-Initiated, Controlled Polymerization of Lactide for the Formulation of Polymeric Nanoparticulate Delivery Vehicles. Angew Chem Int Ed 2008;47:4830-4.

[341] Tong R, Cheng J. Ring-Opening Polymerization-Mediated Controlled Formulation of Polylactide-Drug Nanoparticles. J Am Chem Soc 2009;131:4744-54.

[342] Tong R, Cheng J. Controlled Synthesis of Camptothecin-Polylactide Conjugates and Nanoconjugates. Bioconjugate Chem 2010;21:111-21.

[343] Chen Z, Zhang P, Cheetham AG, Moon JH, Moxley Jr JW, Lin YA, Cui H. Controlled release of free doxorubicin from peptide-drug conjugates by drug loading. J Controlled Release 2014;191:123-30.

[344] Weiner GJ. Building better monoclonal antibody-based therapeutics. Nat Rev Cancer 2015;15:361-70.

[345] Otsuka H, Nagasaki Y, Kataoka K. PEGylated nanoparticles for biological and pharmaceutical applications. Adv Drug Del Rev 2012;64:246-55.

[346] Li D, Ding JX, Tang ZH, Sun H, Zhuang XL, Xu JZ, Chen XS. In vitro evaluation of anticancer nanomedicines based on doxorubicin and amphiphilic Y-shaped copolymers. Int J Nanomedicine 2012;7:2687-97.

[347] Ding JX, Chen JJ, Li D, Xiao CS, Zhang JC, He CL, Zhuang XL, Chen XS. Biocompatible reduction-responsive polypeptide micelles as nanocarriers for enhanced chemotherapy efficacy in vitro. J Mater Chem B Mater Biol Med 2013;1:69-81.

[348] Kataoka K, Harada A, Nagasaki Y. Block copolymer micelles for drug delivery: Design, characterization and biological significance. Adv Drug Del Rev 2012;64:37-48.

[349] Gong J, Chen MW, Zheng Y, Wang SP, Wang YT. Polymeric micelles drug delivery system in oncology. J Controlled Release 2012;159:312-23. 
[350] Ding JX, Xiao CS, He CL, Li MQ, Li D, Zhuang XL, Chen XS. Facile preparation of a cationic poly(amino acid) vesicle for potential drug and gene co-delivery. Nanotechnology 2011;22:494012/1-9.

[351] Yu H, Tang Z, Li M, Song W, Zhang D, Zhang Y, Yang Y, Sun H, Deng M, Chen X. Cisplatin loaded poly(L-glutamic acid)-g-methoxy poly(ethylene glycol) complex nanoparticles for potential cancer therapy: preparation, in vitro and in vivo evaluation. $\mathbf{J}$ Biomed Nanotechnol 2016;12:69-78.

[352] Song W, Tang Z, Zhang D, Zhang Y, Yu H, Li M, Lv S, Sun H, Deng M, Chen X. Anti-tumor efficacy of c(RGDfK)-decorated polypeptide-based micelles co-loaded with docetaxel and cisplatin. Biomaterials 2014;35:3005-14.

[353] Li D, Sun H, Ding J, Tang Z, Zhang Y, Xu W, Zhuang X, Chen X. Polymeric topology and composition constrained polyether-polyester micelles for directional antitumor drug delivery. Acta Biomater 2013;9:8875-84.

[354] Song W, Tang Z, Li M, Lv S, Sun H, Deng M, Liu H, Chen X. Polypeptide-based combination of paclitaxel and cisplatin for enhanced chemotherapy efficacy and reduced side-effects. Acta Biomater 2014;10:1392-402.

[355] Song W, Tang Z, Zhang D, Li M, Gu J, Chen X. A cooperative polymeric platform for tumor-targeted drug delivery. Chem Sci 2016;7:728-36.

[356] Kim S, Shi YZ, Kim JY, Park K, Cheng JX. Overcoming the barriers in micellar drug delivery: loading efficiency, in vivo stability, and micelle-cell interaction. Expert Opin Drug Deliv 2010;7:49-62.

[357] Lee KS, Chung HC, Im SA, Park YH, Kim CS, Kim SB, Rha SY, Lee MY, Ro J. Multicenter phase II trial of Genexol-PM, a Cremophor-free, polymeric micelle formulation of paclitaxel, in patients with metastatic breast cancer. Breast Cancer Res Treat 2008;108:241-50. 
[358] Kim DW, Kim SY, Kim HK, Kim SW, Shin SW, Kim JS, Park K, Lee MY, Heo DS. Multicenter phase II trial of Genexol-PM, a novel Cremophor-free, polymeric micelle formulation of paclitaxel, with cisplatin in patients with advanced non-small-cell lung cancer. Ann Oncol 2007;18:2009-14.

[359] Valle JW, Armstrong A, Newman C, Alakhov V, Pietrzynski G, Brewer J, Campbell S, Corrie P, Rowinsky EK, Ranson M. A phase 2 study of SP1049C, doxorubicin in P-glycoprotein-targeting pluronics, in patients with advanced adenocarcinoma of the esophagus and gastroesophageal junction. Invest New Drugs 2011;29:1029-37.

[360] Von Hoff DD, Mita M, Eisenberg P, LoRusso P, Weiss G, Sachdev J, Mita A, Low S, Hrkach J, Summa J. Abstract LB-203: A phase I study of BIND-014, a PSMA-targeted nanoparticle containing docetaxel, in patients with refractory solid tumors. Cancer Res 2013;73:Abstr LB-203.

[361] Louage B, Zhang Q, Vanparijs N, Voorhaar L, Vande Casteele S, Shi Y, Hennink WE, Van Bocxlaer J, Hoogenboom R, De Geest BG. Degradable Ketal-Based Block Copolymer Nanoparticles for Anticancer Drug Delivery: A Systematic Evaluation. Biomacromolecules 2014;16:336-50.

[362] Plummer R, Wilson R, Calvert H, Boddy A, Griffin M, Sludden J, Tilby M, Eatock M, Pearson D, Ottley C. A Phase I clinical study of cisplatin-incorporated polymeric micelles (NC-6004) in patients with solid tumours. Br J Cancer 2011;104:593-8.

[363] Kato K, Chin K, Yoshikawa T, Yamaguchi K, Tsuji Y, Esaki T, Sakai K, Kimura M, Hamaguchi T, Shimada Y, Matsumura Y, Ikeda R. Phase II study of NK105, a paclitaxel-incorporating micellar nanoparticle, for previously treated advanced or recurrent gastric cancer. Invest New Drugs 2012;30:1621-7.

[364] Li Y, Gao GH, Lee DS. Stimulus-Sensitive Polymeric Nanoparticles and Their Applications as Drug and Gene Carriers. Adv Healthc Mater 2013;2:388-417. 
[365] Ding JX, Zhao L, Li D, Xiao CS, Zhuang XL, Chen XS. Thermo-responsive "hairy-rod" polypeptides for smart antitumor drug delivery. Polym Chem 2013;4:3345-56.

[366] Wei H, Zhuo RX, Zhang XZ. Design and development of polymeric micelles with cleavable links for intracellular drug delivery. Prog Polym Sci 2013;38:503-35.

[367] Ding J, Xiao C, Li Y, Cheng Y, Wang N, He C, Zhuang X, Zhu X, Chen X. Efficacious hepatoma-targeted nanomedicine self-assembled from galactopeptide and doxorubicin driven by two-stage physical interactions. J Controlled Release 2013;169:193-203.

[368] Felber AE, Dufresne MH, Leroux JC. pH-sensitive vesicles, polymeric micelles, and nanospheres prepared with polycarboxylates. Adv Drug Del Rev 2012;64:979-92.

[369] Kiparissides C, Kammona O. Nanoscale carriers for targeted delivery of drugs and therapeutic biomolecules. Can J Chem Eng 2013;91:638-51.

[370] Ding JX, Xiao CS, Yan LS, Tang ZH, Zhuang XL, Chen XS, Jing XB. pH and dual redox responsive nanogel based on poly(l-glutamic acid) as potential intracellular drug carrier. $\mathbf{J}$ Controlled Release 2011;152 (Suppl 1):E11-E13.

[371] Ding J, Shi F, Li D, Chen L, Zhuang X, Chen X. Enhanced endocytosis of acid-sensitive doxorubicin derivatives with intelligent nanogel for improved security and efficacy. Biomater Sci 2013;1:633-46.

[372] Oh JK, Drumright R, Siegwart DJ, Matyjaszewski K. The development of microgels/nanogels for drug delivery applications. Prog Polym Sci 2008;33:448-77.

[373] Kumbar SG, Nair LS, Bhattacharyya S, Laurencin CT. Polymeric nanofibers as novel carriers for the delivery of therapeutic molecules. J Nanosci Nanotechno 2006;6:2591-607.

[374] Toncheva A, Paneva D, Manolova N, Rashkov I, Mita L, Crispi S, Damiano GM. Dual vs. single spinneret electrospinning for the preparation of dual drug containing non-woven fibrous materials. Colloids Surf Physicochem Eng Aspects 2013;439:176-83.

[375] Briggs T, Arinzeh TL. Examining the formulation of emulsion electrospinning for 
improving the release of bioactive proteins from electrospun fibers. J Biomed Mater Res A 2014;102:674-84.

[376] Zhang H, Lou S, Williams GR, Branford-White C, Nie H, Quan J, Zhu LM. A systematic study of captopril-loaded polyester fiber mats prepared by electrospinning. Int J Pharm 2012;439:100-8.

[377] Singh D, Dubey P, Pradhan M, Singh MR. Ceramic nanocarriers: versatile nanosystem for protein and peptide delivery. Expert Opin Drug Deliv 2013;10:241-59.

[378] Kontos S, Hubbell JA. Drug development: longer-lived proteins. Chem Soc Rev 2012;41:2686-95.

[379] Gao WP, Liu WG, Mackay JA, Zalutsky MR, Toone EJ, Chilkoti A. In situ growth of a stoichiometric PEG-like conjugate at a protein's N-terminus with significantly improved pharmacokinetics. Proc Natl Acad Sci USA 2009;106:15231-6.

[380] Gao WP, Liu WG, Christensen T, Zalutsky MR, Chilkoti A. In situ growth of a PEG-like polymer from the $\mathrm{C}$ terminus of an intein fusion protein improves pharmacokinetics and tumor accumulation. Proc Natl Acad Sci USA 2010;107:16432-7.

[381] Vieira J, Clark MA, Shorr RGL, Abuchowski A, Goddard D. PEG adenosine deaminase as a therapeutic adjuvant in the treatment of human acquired immunodeficiency syndrome (AIDS). FASEB J 1996;10:755-755.

[382] Advani A, Earl M, Bleyer A, Douer D, Rytting M, Bleyer A. Toxicities of intravenous (IV) pegasparaginase (ONCASPAR (R)) in adults with acute lymphoblastic leukemia (ALL). Blood 2007;110:827a-827a.

[383] Toy EL, Vekeman F, Laliberte F, Dority B, Perlman D, Barghout V, Duh MS, Heaney ML. Comparison of Infection-Related Hospitalization Risk and Associated Costs among Patients Receiving Sargramostim (Leukine (R)), Filgrastim (Neupogen (R)), and Pegfilgrastim (Neulasta (R)) for Chemotherapy-Induced Neutropenia. Blood 
2008;112:248-9.

[384] Druyts E, Thorlund K, Wu P, Kanters S, Yaya S, Cooper CL, Mills EJ. Efficacy and safety of pegylated interferon alfa-2a or alfa- $2 b$ plus ribavirin for the treatment of chronic hepatitis $\mathrm{C}$ in children and adolescents: a systematic review and meta-analysis. Clin Infect Dis 2013;56:961-7.

[385] Vicent MJ, Duncan R. Polymer conjugates: nanosized medicines for treating cancer. Trends Biotechnol 2006;24:39-47.

[386] Schreiber I, Buchfelder M, Droste M, Forssmann K, Mann K, Saller B, Strasburger CJ. Treatment of acromegaly with the GH receptor antagonist pegvisomant in clinical practice: Safety and efficacy evaluation from the German Pegvisomant Observational Study. Eur J Endocrinol 2007;156:75-82.

[387] Connock M, Tubeuf S, Malottki K, Uthman A, Round J, Bayliss S, Meads C, Moore D. Certolizumab pegol (CIMZIA (R)) for the treatment of rheumatoid arthritis. Health Technol Assess 2010;14:1-10.

[388] De Martino MC, Auriemma RS, Brevetti G, Vitale G, Schiano V, Galdiero M, Grasso L, Lombardi G, Colao A, Pivonello R. The treatment with growth hormone receptor antagonist in acromegaly: Effect on vascular structure and function in patients resistant to somatostatin analogues. J Endocrinol Invest 2010;33:663-70.

[389] Cameron SJ, Gerhardt G, Engelstad M, Young MA, Norris EJ, Sokoll LJ. Interference in clinical chemistry assays by the hemoglobin-based oxygen carrier, Hemospan (R). Clin Biochem 2009;42:221-4.

[390] Gupta S, Bomalaski JS, Carver PD, Hansel DE. Argininosuccinate Synthetase Deficiency as a Possible Therapeutic Indicator for Pegylated Arginine Deiminase (ADI-PEG20) Therapy in Bladder Cancer. Lab Invest 2012;92:210A-1A.

[391] Mueller C, Al-Batran S, Derigs H, Bausch M, Unger C, Jaeger E. Results from a phase IIA 
study of pegylated glutaminase (PEG-PGA) plus 6-diazo-5-oxo-L-norleucine (DON) in patients with metastatic colorectal cancer (mCRC). Ann Oncol 2008;19:90-90.

[392] Ishii H, Furuse J, Nagase M, Maru Y, Yoshino M, Hayashi T. A phase I study of hepatic arterial infusion chemotherapy with zinostatin stimalamer alone for hepatocellular carcinoma. Jpn J Clin Oncol 2003;33:570-3.

[393] Cavazzana-Calvo M, Hacein-Bey S, de Saint Basile G, Gross F, Yvon E, Nusbaum P, Selz F, Hue C, Certain S, Casanova JL, Bousso P, Deist FL, Fischer A. Gene therapy of human severe combined immunodeficiency (SCID)-X1 disease. Science 2000;288:669-72.

[394] Olefsky JM. Diabetes. Gene therapy for rats and mice. Nature 2000;408:420-1.

[395] Kendirci M, Teloken PE, Champion HC, Hellstrom WJG, Bivalacqua TJ. Gene therapy for erectile dysfunction: fact or fiction? Eur Urol 2006;50:1208-22.

[396] Kaplitt MG, Feigin A, Tang C, Fitzsimons HL, Mattis P, Lawlor PA, Bland RJ, Young D, Strybing K, Eidelberg D, During MJ. Safety and tolerability of gene therapy with an adeno-associated virus (AAV) borne GAD gene for Parkinson's disease: an open label, phase I trial. Lancet 2007;369:2097-105.

[397] Zhang S, Zhao B, Jiang H, Wang B, Ma B. Cationic lipids and polymers mediated vectors for delivery of siRNA. J Controlled Release 2007;123:1-10.

[398] Dong X, Lin L, Chen J, Guo Z, Tian H, Li Y, Wei Y, Chen X. A serum-tolerant hydroxyl-modified polyethylenimine as versatile carriers of pDNA/siRNA. Macromol Biosci 2013;13:512-22.

[399] Lehrman S. Virus treatment questioned after gene therapy death. Nature 1999;401:517-8.

[400] Mintzer MA, Simanek EE. Nonviral vectors for gene delivery. Chem Rev 2009;109:259-302.

[401] Tian HY, Tang ZH, Zhuang XL, Chen XS, Jing XB. Biodegradable synthetic polymers: Preparation, functionalization and biomedical application. Prog Polym Sci 
2012;37:237-80.

[402] Dong X, Tian HY, Chen L, Chen J, Chen XS. Biodegradable mPEG-b-P(MCC-g-OEI) copolymers for efficient gene delivery. J Controlled Release 2011;152:135-42.

[403] Tros de Ilarduya C, Sun Y, Duzgunes N. Gene delivery by lipoplexes and polyplexes. Eur J Pharm Sci 2010;40:159-70.

[404] Wang W, Li W, Ma N, Steinhoff G. Non-viral gene delivery methods. Curr Pharm Biotechnol 2013;14:46-60.

[405] Dong X, Tian HY, Chen J, Xia JL, Chen XS, Wei Y. Synthesis and Characterization of Genipin Cross-Linked Oligoethylenimine for Gene Delivery. Acta Polym Sin 2011:1086-91.

[406] Tian HY, Xiong W, Wei JZ, Wang Y, Chen XS, Jing XB, Zhu QY. Gene transfection of hyperbranched PEI grafted by hydrophobic amino acid segment PBLG. Biomaterials 2007;28:2899-907.

[407] Wen YT, Pan SR, Luo X, Zhang X, Zhang W, Feng M. A biodegradable low molecular weight polyethylenimine derivative as low toxicity and efficient gene vector. Bioconjugate Chem 2009;20:322-32.

[408] Dong X, Lin L, Chen J, Tian H, Xiao C, Guo Z, Li Y, Wei Y, Chen X. Multi-armed poly(aspartate-g-OEI) copolymers as versatile carriers of pDNA/siRNA. Acta Biomater 2013;9:6943-52.

[409] Wu GY, Wu CH. Receptor-mediated in vitro gene transformation by a soluble DNA carrier system. J Biol Chem 1987;262:4429-32.

[410] Wagner E, Plank C, Zatloukal K, Cotten M, Birnstiel ML. Influenza virus hemagglutinin HA-2 N-terminal fusogenic peptides augment gene transfer by transferrin-polylysine-DNA complexes: toward a synthetic virus-like gene-transfer vehicle. Proc Natl Acad Sci USA 1992;89:7934-8. 
[411] Kakizawa Y, Harada A, Kataoka K. Glutathione-sensitive stabilization of block copolymer micelles composed of antisense DNA and thiolated poly(ethylene glycol)-block-poly(L-lysine): a potential carrier for systemic delivery of antisense DNA. Biomacromolecules 2001;2:491-7.

[412] Fukushima S, Miyata K, Nishiyama N, Kanayama N, Yamasaki Y, Kataoka K. PEGylated polyplex micelles from triblock catiomers with spatially ordered layering of condensed pDNA and buffering units for enhanced intracellular gene delivery. J Am Chem Soc 2005;127:2810-1.

[413] Marano RJ, Rakoczy PE. Treatments for choroidal and retinal neovascularization: a focus on oligonucleotide therapy and delivery for the regulation of gene function. Clin Experiment Ophthalmol 2005;33:81-9.

[414] Dai H, Jiang X, Tan GC, Chen Y, Torbenson M, Leong KW, Mao HQ. Chitosan-DNA nanoparticles delivered by intrabiliary infusion enhance liver-targeted gene delivery. Int $\mathrm{J}$ Nanomedicine 2006;1:507-22.

[415] Green JJ, Langer R, Anderson DG. A combinatorial polymer library approach yields insight into nonviral gene delivery. Acc Chem Res 2008;41:749-59.

[416] Fields RJ, Cheng CJ, Quijano E, Weller C, Kristofik N, Duong N, Hoimes C, Egan ME, Saltzman WM. Surface modified poly(beta amino ester)-containing nanoparticles for plasmid DNA delivery. J Controlled Release 2012;164:41-8.

[417] Cutlar L, Zhou DZ, Gao YS, Zhao TY, Greiser U, Wang W, Wang WX. Highly Branched Poly(beta-Amino Esters): Synthesis and Application in Gene Delivery.

Biomacromolecules 2015;16:2609-17.

[418] Gabrielson NP, Lu H, Yin LC, Li D, Wang F, Cheng JJ. Reactive and Bioactive Cationic a-Helical Polypeptide Template for Nonviral Gene Delivery. Angew Chem Int Ed 2012;51:1143-7. 
[419] Zheng N, Song ZY, Liu Y, Zhang RJ, Zhang RY, Yao C, Uckun FM, Yin LC, Cheng JJ. Redox-responsive, reversibly-crosslinked thiolated cationic helical polypeptides for efficient siRNA encapsulation and delivery. J Controlled Release 2015;205:231-9.

[420] Zhang RJ, Zheng N, Song ZY, Yin LC, Cheng JJ. The effect of side-chain functionality and hydrophobicity on the gene delivery capabilities of cationic helical polypeptides. Biomaterials 2014;35:3443-54.

[421] Chen CK, Jones CH, Mistriotis P, Yu Y, Ma XN, Ravikrishnan A, Jiang M, Andreadis ST, Pfeifer BA, Cheng C. Poly(ethylene glycol)-block-cationic polylactide nanocomplexes of differing charge density for gene delivery. Biomaterials 2013;34:9688-99.

[422] Li WJ, Szoka FC. Lipid-based nanoparticles for nucleic acid delivery. Pharm Res 2007;24:438-49.

[423] Lasic DD, Templeton NS. Liposomes in gene therapy. Adv Drug Del Rev 1996;20:221-66.

[424] Lonez C, Vandenbranden M, Ruysschaert JM. Cationic liposomal lipids: From gene carriers to cell signaling. Prog Lipid Res 2008;47:340-7.

[425] Hurley CA, Wong JB, Ho J, Writer M, Irvine SA, Lawrence MJ, Hart SL, Tabor AB, Hailes HC. Mono- and dicationic short PEG and methylene dioxyalkylglycerols for use in synthetic gene delivery systems. Org Biomol Chem 2008;6:2554-9.

[426] Malone RW, Felgner PL, Verma IM. Cationic liposome-mediated RNA transfection. Proc Natl Acad Sci USA 1989;86:6077-81.

[427] Judge AD, Robbins M, Tavakoli I, Levi J, Hu L, Fronda A, Ambegia E, McClintock K, MacLachlan I. Confirming the RNAi-mediated mechanism of action of siRNA-based cancer therapeutics in mice. J Clin Invest 2009;119:661-73.

[428] Fillion P, Desjardins A, Sayasith K, Lagace J. Encapsulation of DNA in negatively charged liposomes and inhibition of bacterial gene expression with fluid liposome-encapsulated antisense oligonucleotides. Biochim Biophys Acta - biomembranes 2001;1515:44-54. 
[429] Matsumoto S, Christie RJ, Nishiyama N, Miyata K, Ishii A, Oba M, Koyama H, Yamasaki Y, Kataoka K. Environment-responsive block copolymer micelles with a disulfide cross-linked core for enhanced siRNA delivery. Biomacromolecules 2009;10:119-27.

[430] Osada K, Christie RJ, Kataoka K. Polymeric micelles from poly(ethylene glycol)-poly(amino acid) block copolymer for drug and gene delivery. J R Soc Interface 2009;6:S325-S39.

[431] Kunath K, von Harpe A, Petersen H, Fischer D, Voigt K, Kissel T, Bickel U. The structure of PEG-modified poly(ethylene imines) influences biodistribution and pharmacokinetics of their complexes with NF-kappa B decoy in mice. Pharm Res 2002;19:810-7.

[432] Zheng CF, Zheng MB, Gong P, Deng JZ, Yi HQ, Zhang PF, Zhang YJ, Liu P, Ma YF, Cai LT. Polypeptide cationic micelles mediated co-delivery of docetaxel and siRNA for synergistic tumor therapy. Biomaterials 2013;34:3431-8.

[433] Sun TM, Du JZ, Yao YD, Mao CQ, Dou S, Huang SY, Zhang PZ, Leong KW, Song EW, Wang J. Simultaneous delivery of siRNA and paclitaxel via a "two-in-one" micelleplex promotes synergistic tumor suppression. ACS Nano 2011;5:1483-94.

[434] Cheng D, Cao N, Chen JF, Yu XS, Shuai XT. Multifunctional nanocarrier mediated co-delivery of doxorubicin and siRNA for synergistic enhancement of glioma apoptosis in rat. Biomaterials 2012;33:1170-9.

[435] Khan M, Ong ZY, Wiradharma N, Attia ABE, Yang YY. Advanced Materials for Co-Delivery of Drugs and Genes in Cancer Therapy. Adv Healthc Mater 2012;1:373-92.

[436] Creixell M, Peppas NA. Co-delivery of siRNA and therapeutic agents using nanocarriers to overcome cancer resistance. Nano Today 2012;7:367-79.

[437] Yu BJ, Tang C, Yin CH. Enhanced antitumor efficacy of folate modified amphiphilic nanoparticles through co-delivery of chemotherapeutic drugs and genes. Biomaterials 2014;35:6369-78. 
[438] Li ZR, Li JG, Huang JS, Zhang JJ, Cheng D, Shuai XT. Synthesis and Characterization of pH-Responsive Copolypeptides Vesicles for siRNA and Chemotherapeutic Drug Co-Delivery. Macromol Biosci 2015;15:1497-506.

[439] Wang Y, Gao SJ, Ye WH, Yoon HS, Yang YY. Co-delivery of drugs and DNA from cationic core-shell nanoparticles self-assembled from a biodegradable copolymer. Nature Mater 2006;5:791-6.

[440] Garbuzenko OB, Saad M, Pozharov VP, Reuhl KR, Mainelis G, Minko T. Inhibition of lung tumor growth by complex pulmonary delivery of drugs with oligonucleotides as suppressors of cellular resistance. Proc Natl Acad Sci USA 2010;107:10737-42.

[441] Chen CK, Law WC, Aalinkeel R, Yu Y, Nair B, Wu JC, Mahajan S, Reynolds JL, Li YK, Lai CK, Tzanakakis ES, Schwartz SA, Prasad PN, Cheng C. Biodegradable cationic polymeric nanocapsules for overcoming multidrug resistance and enabling drug-gene co-delivery to cancer cells. Nanoscale 2014;6:1567-72.

[442] Jilek S, Merkle HP, Walter E. DNA-loaded biodegradable microparticles as vaccine delivery systems and their interaction with dendritic cells. Adv Drug Del Rev 2005;57:377-90.

[443] Xu ZP, Zeng QH, Lu GQ, Yu AB. Inorganic nanoparticles as carriers for efficient cellular delivery. Chem Eng Sci 2006;61:1027-40.

[444] Liu YP, Meyer-Zaika W, Franzka S, Schmid G, Tsoli M, Kuhn H. Gold-cluster degradation by the transition of B-DNA into A-DNA and the formation of nanowires. Angew Chem Int Ed 2003;42:2853-7.

[445] Figueroa ER, Lin AY, Yan J, Luo L, Foster AE, Drezek RA. Optimization of PAMAM-gold nanoparticle conjugation for gene therapy. Biomaterials 2014;35:1725-34.

[446] Bahadur K, Thapa B, Bhattarai N. Gold nanoparticle-based gene delivery: promises and challenges. Nanotechnol Rev 2014;3:269-80. 
[447] Kim JH, Park K, Nam HY, Lee S, Kim K, Kwon IC. Polymers for bioimaging. Prog Polym Sci 2007;32:1031-53.

[448] Liu Y, Chen Z, Liu C, Yu D, Lu Z, Zhang N. Gadolinium-loaded polymeric nanoparticles modified with Anti-VEGF as multifunctional MRI contrast agents for the diagnosis of liver cancer. Biomaterials 2011;32:5167-76.

[449] Vivero-Escoto JL, Rieter WJ, Lau H, Huxford-Phillips RC, Lin W. Biodegradable polysilsesquioxane nanoparticles as efficient contrast agents for magnetic resonance imaging. Small 2013;9:3523-31.

[450] Mi P, Cabral H, Kokuryo D, Rafi M, Terada Y, Aoki I, Saga T, Takehiko I, Nishiyama N, Kataoka K. Gd-DTPA-loaded polymer-metal complex micelles with high relaxivity for MR cancer imaging. Biomaterials 2013;34:492-500.

[451] Bock JC, Kaufmann F, Felix R. Comparison of gadolinium-DTPA and macromolecular gadolinium-DTPA-polylysine for contrast-enhanced pulmonary time-of-flight magnetic resonance angiography. Invest Radiol 1996;31:652-7.

[452] Shiraishi K, Kawano K, Minowa T, Maitani Y, Yokoyama M. Preparation and in vivo imaging of PEG-poly(L-lysine)-based polymeric micelle MRI contrast agents. J Controlled Release 2009;136:14-20.

[453] Kim KS, Park W, Hu J, Bae YH, Na K. A cancer-recognizable MRI contrast agents using pH-responsive polymeric micelle. Biomaterials 2014;35:337-43.

[454] Kobayashi H, Kawamoto S, Choyke PL, Sato N, Knopp MV, Star RA, Waldmann TA, Tagaya Y, Brechbiel MW. Comparison of dendrimer-based macromolecular contrast agents for dynamic micro-magnetic resonance lymphangiography. Magn Reson Med 2003;50:758-66.

[455] Xu RZ, Wang YL, Wang XL, Jeong EK, Parker DL, Lu ZR. In Vivo evaluation of a PAMAM-cystamine-(Gd-DO3A) conjugate as a biodegradable macromolecular MRI 
contrast agent. Exp Biol Med 2007;232:1081-9.

[456] Laus S, Sour A, Ruloff R, Tóth É, Merbach AE. Rotational dynamics account for pH-dependent relaxivities of PAMAM dendrimeric, Gd-based potential MRI contrast agents. Chemistry 2005;11:3064-76.

[457] Kobayashi H, Brechbiel MW. Nano-sized MRI contrast agents with dendrimer cores. Adv Drug Del Rev 2005;57:2271-86.

[458] Misselwitz B, Schmitt-Willich H, Ebert W, Frenzel T, Weinmann HJ. Pharmacokinetics of Gadomer-17, a new dendritic magnetic resonance contrast agent. Magn Reson Mater Phys, Biol Med 2001;12:128-34.

[459] Zhu Q, Qiu F, Zhu BS, Zhu XY. Hyperbranched polymers for bioimaging. Rsc Adv 2013;3:2071-83.

[460] Bashir MR, Bhatti L, Marin D, Nelson RC. Emerging applications for ferumoxytol as a contrast agent in MRI. J Magn Reson Imaging 2015;41:884-98.

[461] Zhang S, Gong M, Zhang D, Yang H, Gao F, Zou L. Thiol-PEG-carboxyl-stabilized Fe 2 O 3/Au nanoparticles targeted to CD105: Synthesis, characterization and application in MR imaging of tumor angiogenesis. Eur J Radiol 2014;83:1190-8.

[462] Gupta AK, Gupta M. Synthesis and surface engineering of iron oxide nanoparticles for biomedical applications. Biomaterials 2005;26:3995-4021.

[463] Jung CW, Jacobs P. Physical and chemical properties of superparamagnetic iron oxide MR contrast agents: Ferumoxides, ferumoxtran, ferumoxsil. Magn Reson Imaging 1995;13:661-74.

[464] Briley-Saebo KC, Johansson LO, Hustvedt SO, Haldorsen AG, Bjornerud A, Fayad ZA, Ahlstrom HK. Clearance of iron oxide particles in rat liver: effect of hydrated particle size and coating material on liver metabolism. Invest Radiol 2006;41:560-71.

[465] Shan GB, Xing JM, Luo MF, Liu HZ, Chen JY. Immobilization of Pseudomonas 
delafieldii with magnetic polyvinyl alcohol beads and its application in biodesulfurization. Biotechnol Lett 2003;25:1977-81.

[466] Zhang Y, Kohler N, Zhang MQ. Surface modification of superparamagnetic magnetite nanoparticles and their intracellular uptake. Biomaterials 2002;23:1553-61.

[467] Wang YX. Superparamagnetic iron oxide based MRI contrast agents: Current status of clinical application. Quant Imaging Med Surg 2011;1:35-40.

[468] Paulus MJ, Gleason SS, Kennel SJ, Hunsicker PR, Johnson DK. High resolution X-ray computed tomography: An emerging tool for small animal cancer research. Neoplasia 2000;2:62-70.

[469] Liu Y, Ai K, Lu L. Nanoparticulate X-ray computed tomography contrast agents: from design validation to in vivo applications. Acc Chem Res 2012;45:1817-27.

[470] Yu SB, Watson AD. Metal-based X-ray contrast media. Chem Rev 1999;99:2353-77.

[471] Shilo M, Reuveni T, Motiei M, Popovtzer R. Nanoparticles as computed tomography contrast agents: current status and future perspectives. Nanomedicine 2012;7:257-69.

[472] Leng H, Wang X, Ross RD, Niebur GL, Roeder RK. Micro-computed tomography of fatigue microdamage in cortical bone using a barium sulfate contrast agent. J Mech Behav Biomed Mater 2008;1:68-75.

[473] Yin Q, Yap FY, Yin L, Ma L, Zhou Q, Dobrucki LW, Fan TM, Gaba RC, Cheng J. Poly(iohexol) nanoparticles as contrast agents for in vivo X-ray computed tomography imaging. J Am Chem Soc 2013;135:13620-3.

[474] Ai K, Liu Y, Liu J, Yuan Q, He Y, Lu L. Large-Scale Synthesis of Bi2S3 Nanodots as a Contrast Agent for In Vivo X-ray Computed Tomography Imaging. Adv Mater 2011;23:4886-91.

[475] Rabin O, Manuel Perez J, Grimm J, Wojtkiewicz G, Weissleder R. An X-ray computed tomography imaging agent based on long-circulating bismuth sulphide nanoparticles. 
Nature Mater 2006;5:118-22.

[476] Liu Y, Ai K, Liu J, Yuan Q, He Y, Lu L. A high-performance ytterbium-based nanoparticulate contrast agent for in vivo X-ray computed tomography imaging. Angew Chem Int Ed 2012;51:1437-42.

[477] Lusic H, Grinstaff MW. X-ray-computed tomography contrast agents. Chem Rev 2012;113:1641-66.

[478] de Vries A, Custers E, Lub J, van den Bosch S, Nicolay K, Grüll H. Block-copolymer-stabilized iodinated emulsions for use as CT contrast agents. Biomaterials 2010;31:6537-44.

[479] Hallouard F, Anton N, Choquet P, Constantinesco A, Vandamme T. Iodinated blood pool contrast media for preclinical X-ray imaging applications - A review. Biomaterials 2010;31:6249-68.

[480] Hyafil F, Cornily JC, Feig JE, Gordon R, Vucic E, Amirbekian V, Fisher EA, Fuster V, Feldman LJ, Fayad ZA. Noninvasive detection of macrophages using a nanoparticulate contrast agent for computed tomography. Nat Med 2007;13:636-41.

[481] Ho Kong W, Jae Lee W, Yun Cui Z, Hyun Bae K, Gwan Park T, Hoon Kim J, Park K, Won Seo S. Nanoparticulate carrier containing water-insoluble iodinated oil as a multifunctional contrast agent for computed tomography imaging. Biomaterials 2007;28:5555-61.

[482] Jain S, Hirst DG, O'Sullivan JM. Gold nanoparticles as novel agents for cancer therapy. Br J Radiol 2012;85:101-13.

[483] Cai QY, Kim SH, Choi KS, Kim SY, Byun SJ, Kim KW, Park SH, Juhng SK, Yoon KH. Colloidal gold nanoparticles as a blood-pool contrast agent for x-ray computed tomography in mice. Invest Radiol 2007;42:797-806.

[484] Popovtzer R, Agrawal A, Kotov NA, Popovtzer A, Balter J, Carey TE, Kopelman R. Targeted Gold Nanoparticles Enable Molecular CT Imaging of Cancer. Nano Lett 
2008;8:4593-6.

[485] Wheatley MA. Polymeric Vesicles/Capsules for Diagnostic Applications in Medicine. In: Broz P, editor. Polymer-based Nanostructures: Medical Applications. 1st ed: The Royal Society of Chemistry; 2010. p. 237-58.

[486] Eck W, Nicholson AI, Zentgraf H, Semmler W, Bartling Sn. Anti-CD4-targeted gold nanoparticles induce specific contrast enhancement of peripheral lymph nodes in X-ray computed tomography of live mice. Nano Lett 2010;10:2318-22.

[487] Kim D, Park S, Lee JH, Jeong YY, Jon S. Antibiofouling polymer-coated gold nanoparticles as a contrast agent for in vivo X-ray computed tomography imaging. J Am Chem Soc 2007;129:7661-5.

[488] Sun IC, Eun DK, Koo H, Ko CY, Kim HS, Yi DK, Choi K, Kwon IC, Kim K, Ahn CH. Tumor-targeting gold particles for dual computed tomography/optical cancer imaging. Angew Chem Int Ed Engl 2011;123:9520-3.

[489] Kim D, Jeong YY, Jon S. A drug-loaded aptamer-gold nanoparticle bioconjugate for combined CT imaging and therapy of prostate cancer. ACS Nano 2010;4:3689-96.

[490] Liu Y, Ai K, Liu J, Yuan Q, He Y, Lu L. Hybrid BaYbF(5) nanoparticles: novel binary contrast agent for high-resolution in vivo X-ray computed tomography angiography. Adv Healthc Mater 2012;1:461-6.

[491] Licha K, Hessenius C, Becker A, Henklein P, Bauer M, Wisniewski S, Wiedenmann B, Semmler W. Synthesis, characterization, and biological properties of cyanine-labeled somatostatin analogues as receptor-targeted fluorescent probes. Bioconjugate Chem 2001;12:44-50.

[492] Funovics M, Weissleder R, Tung CH. Protease sensors for bioimaging. Anal Bioanal Chem 2003;377:956-63.

[493] Guo Z, Park S, Yoon J, Shin I. Recent progress in the development of near-infrared 
fluorescent probes for bioimaging applications. Chem Soc Rev 2014;43:16-29.

[494] Lee S, Ryu JH, Park K, Lee A, Lee SY, Youn IC, Ahn CH, Yoon SM, Myung SJ, Moon DH, Chen X, Choi K, Kwon IC, Kim K. Polymeric nanoparticle-based activatable near-infrared nanosensor for protease determination in vivo. Nano Lett 2009;9:4412-6.

[495] Luo S, Zhang E, Su Y, Cheng T, Shi C. A review of NIR dyes in cancer targeting and imaging. Biomaterials 2011;32:7127-38.

[496] Adjili S, Favier A, Fargier G, Thomas A, Massin J, Monier K, Favard C, Vanbelle C, Bruneau S, Peyrieras N, Andraud C, Muriaux D, Charreyre MT. Biocompatible photoresistant far-red emitting, fluorescent polymer probes, with near-infrared two-photon absorption, for living cell and zebrafish embryo imaging. Biomaterials 2015;46:70-81.

[497] Hong G, Zou Y, Antaris AL, Diao S, Wu D, Cheng K, Zhang X, Chen C, Liu B, He Y, Wu JZ, Yuan J, Zhang B, Tao Z, Fukunaga C, Dai H. Ultrafast fluorescence imaging in vivo with conjugated polymer fluorophores in the second near-infrared window. Nat Commun $2014 ; 5: 4206 / 1-9$.

[498] Weissleder R, Tung CH, Mahmood U, Bogdanov A. In vivo imaging of tumors with protease-activated near-infrared fluorescent probes. Nat Biotechnol 1999;17:375-8.

[499] Campo MA, Gabriel D, Kucera P, Gurny R, Lange N. Polymeric Photosensitizer Prodrugs for Photodynamic Therapy. Photochem Photobiol 2007;83:958-65.

[500] Bremer C, Tung CH, Bogdanov A, Weissleder R. Imaging of Differential Protease Expression in Breast Cancers for Detection of Aggressive Tumor Phenotypes. Radiology 2002;222:814-8.

[501] Yan S, Sameni M, Sloane BF. Cathepsin B and human tumor progression. Biol Chem 1998;379:113-23.

[502] Gondi CS, Rao JS. Cathepsin B as a cancer target. Expert Opin Ther Targets 2013;17:281-91. 
[503] Hashimoto Y, Kakegawa H, Narita Y, Hachiya Y, Hayakawa T, Kos J, Turk V, Katunuma N. Significance of Cathepsin B Accumulation in Synovial Fluid of Rheumatoid Arthritis. Biochem Biophys Res Commun 2001;283:334-9.

[504] Chen J, Tung CH, Mahmood U, Ntziachristos V, Gyurko R, Fishman MC, Huang PL, Weissleder R. In Vivo Imaging of Proteolytic Activity in Atherosclerosis. Circulation 2002;105:2766-71.

[505] Jaffer FA, Kim DE, Quinti L, Tung CH, Aikawa E, Pande AN, Kohler RH, Shi GP, Libby P, Weissleder R. Optical visualization of cathepsin K activity in atherosclerosis with a novel, protease-activatable fluorescence sensor. Circulation 2007;115:2292-8.

[506] Jaffer FA, Tung CH, Gerszten RE, Weissleder R. In vivo Imaging of thrombin activity in experimental thrombi with thrombin-sensitive near-infrared molecular probe. Arterioscler Thromb Vasc Biol 2002;22:1929-35.

[507] Jaffer FA, Tung CH, Wykrzykowska JJ, Ho NH, Houng AK, Reed GL, Weissleder R. Molecular imaging of factor XIIIa activity in thrombosis using a novel, near-infrared fluorescent contrast agent that covalently links to thrombi. Circulation 2004;110:170-6.

[508] Kim K, Lee M, Park H, Kim JH, Kim S, Chung H, Choi K, Kim IS, Seong BL, Kwon IC. Cell-permeable and biocompatible polymeric nanoparticles for apoptosis imaging. $\mathrm{J} \mathrm{Am}$ Chem Soc 2006;128:3490-1.

[509] Zhang XX, Eden HS, Chen X. Peptides in cancer nanomedicine: Drug carriers, targeting ligands and protease substrates. J Controlled Release 2012;159:2-13.

[510] Lee S, Park K, Kim K, Choi K, Kwon IC. Activatable imaging probes with amplified fluorescent signals. Chem Commun 2008:4250-60.

[511] Pelley JL, Daar AS, Saner MA. State of academic knowledge on toxicity and biological fate of quantum dots. Toxicol Sci 2009;112:276-96.

[512] Kušić H, Leszczynska D. Altered toxicity of organic pollutants in water originated from 
simultaneous exposure to UV photolysis and CdSe/ZnS quantum dots. Chemosphere 2012;89:900-6.

Wu X, Liu H, Liu J, Haley KN, Treadway JA, Larson JP, Ge N, Peale F, Bruchez MP. Immunofluorescent labeling of cancer marker Her2 and other cellular targets with semiconductor quantum dots. Nat Biotechnol 2003;21:41-6.

[514] Gao X, Cui Y, Levenson RM, Chung LWK, Nie S. In vivo cancer targeting and imaging with semiconductor quantum dots. Nat Biotechnol 2004;22:969-76.

Fernández-Argüelles MT, Yakovlev A, Sperling RA, Luccardini C, Gaillard S, Sanz Medel A, Mallet JM, Brochon JC, Feltz A, Oheim M, Parak WJ. Synthesis and characterization of polymer-coated quantum dots with integrated acceptor dyes as FRET-based nanoprobes. Nano Lett 2007;7:2613-7.

[516] Pellegrino T, Manna L, Kudera S, Liedl T, Koktysh D, Rogach AL, Keller S, Rädler J, Natile G, Parak WJ. Hydrophobic nanocrystals coated with an amphiphilic polymer shell: A general route to water soluble nanocrystals. Nano Lett 2004;4:703-7.

[517] Cai WB, Shin DW, Chen K, Gheysens O, Cao QZ, Wang SX, Gambhir SS, Chen XY. Peptide-labeled near-infrared quantum dots for imaging tumor vasculature in living subjects. Nano Lett 2006;6:669-76.

[518] Ghoroghchian PP, Frail PR, Susumu K, Blessington D, Brannan AK, Bates FS, Chance B, Hammer DA, Therien MJ. Near-infrared-emissive polymersomes: Self-assembled soft matter for in vivo optical imaging. Proc Natl Acad Sci USA 2005;102:2922-7.

[519] Smith WE, Brownell J, White CC, Afsharinejad Z, Tsai J, Hu X, Polyak SJ, Gao X, Kavanagh TJ, Eaton DL. In vitro toxicity assessment of amphiphillic polymer-coated CdSe/ZnS quantum dots in two human liver cell models. ACS Nano 2012;6:9475-84. [520] Speranskaya ES, Beloglazova NV, Lenain P, De Saeger S, Wang Z, Zhang S, Hens Z, Knopp D, Niessner R, Potapkin DV, Goryacheva IY. Polymer-coated fluorescent 
CdSe-based quantum dots for application in immunoassay. Biosens Bioelectron 2014;53:225-31.

[521] Sun G, Xu J, Hagooly A, Rossin R, Li Z, Moore DA, Hawker CJ, Welch MJ, Wooley KL. Strategies for optimized radiolabeling of nanoparticles for in vivo PET Imaging. Adv Mater 2007;19:3157-62.

[522] Anderson CJ, Welch MJ. Radiometal-Labeled Agents (Non-Technetium) for Diagnostic Imaging. Chem Rev 1999;99:2219-34.

[523] Van Den Bossche B, Van de Wiele C. Receptor imaging in oncology by means of nuclear medicine: current status. J Clin Oncol 2004;22:3593-607.

[524] Jensen AI, Binderup T, Kumar EK P, Kjær A, Rasmussen PH, Andresen TL. Positron emission tomography based analysis of long-circulating cross-linked triblock polymeric micelles in a U87MG mouse xenograft model and comparison of DOTA and CB-TE2A as chelators of copper-64. Biomacromolecules 2014;15:1625-33.

[525] Sun X, Rossin R, Turner JL, Becker ML, Joralemon MJ, Welch MJ, Wooley KL. An assessment of the effects of shell cross-linked nanoparticle size, core composition, and surface PEGylation on in vivo biodistribution. Biomacromolecules 2005;6:2541-54.

[526] Rossin R, Pan DPJ, Qi K, Turner JL, Sun XK, Wooley KL, Welch MJ. Cu-64-labeled folate-conjugated shell cross-linked nanoparticles for tumor imaging and radiotherapy: Synthesis, radiolabeling, and biologic evaluation. J Nucl Med 2005;46:1210-8.

[527] Mitra A, Nan A, Line BR, Ghandehari H. Nanocarriers for nuclear imaging and radiotherapy of cancer. Curr Pharm Des 2006;12:4729-49.

[528] Prince JF, Smits ML, Krijger GC, Zonnenberg BA, van den Bosch MA, Nijsen JF, Lam MG. Radiation Emission from Patients Treated with Holmium-166 Radioembolization. J Vasc Interv Radiol 2014;25:1956-63. e1.

[529] Lindenberg L, Adler S, Thomas A, Maltzman J, Wallin B, Mena E, Kurdziel K, Paik C, 
Choyke P, Hassan R. Initial clinical experience of 111In radiolabelled amatuximab in patients with mesothelin-expressing cancers. J Nucl Med 2014;55 (Suppl 1):1138-1138.

[530] Wu H, Lou C, Huang Z, Shi G. SPECT imaging of dopamine transporters with (99m)Tc-TRODAT-1 in major depression and Parkinson's disease. J Neuropsychiatry Clin Neurosci 2011;23:63-7.

[531] Zhang R, Xiong C, Huang M, Zhou M, Huang Q, Wen X, Liang D, Li C.

Peptide-conjugated polymeric micellar nanoparticles for Dual SPECT and optical imaging of EphB4 receptors in prostate cancer xenografts. Biomaterials 2011;32:5872-9.

[532] Srinivasan S, Jayakumar R, Chennazhi KP, Levorson EJ, Mikos AG, Nair SV. Multiscale

Fibrous Scaffolds in Regenerative Medicine. In: Jayakumar R, Nair SV, editors.

Biomedical Applications of Polymeric Nanofibers: Springer Science \& Business Media; 2012. p. 1-20.

[533] Holmes B, Castro NJ, Zhang LG, Zussman E. Electrospun Fibrous Scaffolds for Bone and Cartilage Tissue Generation: Recent Progress and Future Developments. Tissue Eng Part B Rev 2012;18:478-86.

[534] Eap S, Ferrand A, Palomares CM, Hebraud A, Stoltz JF, Mainard D, Schlatter G,

Benkirane-Jessel N. Electrospun nanofibrous 3D scaffold for bone tissue engineering. Bio-Med Mater Eng 2012;22:137-41.

[535] Dahlin RL, Kasper FK, Mikos AG. Polymeric nanofibers in tissue engineering. Tissue Eng Part B Rev 2011;17:349-64.

[536] Buckwalter JA, Glimcher MJ, Cooper RR, Recker R. Bone biology. 1. Structure, blood-supply, cells, matrix, and mineralization. J Bone Joint Surg Am 1995;77A:1256-75.

[537] Hasan A, Memic A, Annabi N, Hossain M, Paul A, Dokmeci MR, Dehghani F, Khademhosseini A. Electrospun scaffolds for tissue engineering of vascular grafts. Acta Biomater 2014;10:11-25. 
[538] Akbarzadeh R, Yousefi AM. Effects of processing parameters in thermally induced phase separation technique on porous architecture of scaffolds for bone tissue engineering. $\mathbf{J}$ Biomed Mater Res B Appl Biomater 2014;102:1304-15.

[539] He B, Yuan X, Zhou A, Zhang H, Jiang D. Designer functionalised self-assembling peptide nanofibre scaffolds for cartilage tissue engineering. Expert Rev Mol Med 2014;16:e12/1-9.

[540] Yoshimoto H, Shin YM, Terai H, Vacanti JP. A biodegradable nanofiber scaffold by electrospinning and its potential for bone tissue engineering. Biomaterials $2003 ; 24: 2077-82$.

[541] Ruckh TT, Kumar K, Kipper MJ, Popat KC. Osteogenic differentiation of bone marrow stromal cells on poly(epsilon-caprolactone) nanofiber scaffolds. Acta Biomater 2010;6:2949-59.

[542] Shin M, Yoshimoto H, Vacanti JP. In vivo bone tissue engineering using mesenchymal stem cells on a novel electrospun nanofibrous scaffold. Tissue Eng 2004;10:33-41.

[543] Hartgerink JD, Beniash E, Stupp SI. Self-assembly and mineralization of peptide-amphiphile nanofibers. Science 2001;294:1684-8.

[544] Zhang YZ, Reddy VJ, Wong SY, Li X, Su B, Ramakrishna S, Lim CT. Enhanced biomineralization in osteoblasts on a novel electrospun biocomposite nanofibrous substrate of hydroxyapatite/collagen/chitosan. Tissue engineering Part A 2010;16:1949-60.

[545] Li CM, Vepari C, Jin HJ, Kim HJ, Kaplan DL. Electrospun silk-BMP-2 scaffolds for bone tissue engineering. Biomaterials 2006;27:3115-24.

[546] Tautzenberger A, Kovtun A, Ignatius A. Nanoparticles and their potential for application in bone. Int J Nanomedicine 2012;7:4545-57.

[547] Arora P, Sindhu A, Dilbaghi N, Chaudhury A, Rajakumar G, Rahuman AA. Nano-regenerative medicine towards clinical outcome of stem cell and tissue engineering in humans. J Cell Mol Med 2012;16:1991-2000. 
[548] Allemann E, Gurny R, Doelker E. Drug-loaded nanoparticles: preparation methods and drug targeting issues. Eur J Pharm Biopharm 1993;39:173-91.

[549] Cade D, Ramus E, Rinaudo M, Auzely-Velty R, Delair T, Hamaide T. Tailoring of bioresorbable polymers for elaboration of sugar-functionalized nanoparticles.

Biomacromolecules 2004;5:922-7.

[550] Wang HA, Leeuwenburgh SCG, Li YB, Jansen JA. The use of micro- and nanospheres as functional components for bone tissue regeneration. Tissue Eng Part B Rev 2012;18:24-39.

[551] Temenoff JS, Mikos AG. Review: tissue engineering for regeneration of articular cartilage. Biomaterials 2000;21:431-40.

[552] Li WJ, Danielson KG, Alexander PG, Tuan RS. Biological response of chondrocytes cultured in three-dimensional nanofibrous poly(epsilon-caprolactone) scaffolds. J Biomed Mater Res A 2003;67A:1105-14. da Silva MA, Crawford A, Mundy J, Martins A, Araujo JV, Hatton PV, Reis RL, Neves NM. Evaluation of Extracellular Matrix Formation in Polycaprolactone and Starch-Compounded Polycaprolactone Nanofiber Meshes When Seeded with Bovine Articular Chondrocytes. Tissue engineering Part A 2009;15:377-85.

[554] Li WJ, Tuli R, Huang XX, Laquerriere P, Tuan RS. Multilineage differentiation of human mesenchymal stem cells in a three-dimensional nanofibrous scaffold. Biomaterials 2005;26:5158-66.

[555] Li WJ, Tuli R, Okafor C, Derfoul A, Danielson KG, Hall DJ, Tuan RS. A three-dimensional nanofibrous scaffold for cartilage tissue engineering using human mesenchymal stem cells. Biomaterials 2005;26:599-609.

[556] Santo VE, Gomes ME, Mano JF, Reis RL. From nano- to macro-scale: nanotechnology approaches for spatially controlled delivery of bioactive factors for bone and cartilage engineering. Nanomedicine 2012;7:1045-66. 
[557] Chung E, Ricles LM, Stowers RS, Nam SY, Emelianov SY, Suggs LJ. Multifunctional nanoscale strategies for enhancing and monitoring blood vessel regeneration. Nano Today 2012;7:514-31.

[558] Dvir T, Timko BP, Kohane DS, Langer R. Nanotechnological strategies for engineering complex tissues. Nat Nanotechnol 2011;6:13-22.

[559] Tsang KY, Cheung MCH, Chan D, Cheah KSE. The developmental roles of the extracellular matrix: beyond structure to regulation. Cell Tissue Res 2010;339:93-110.

[560] Zhang G, Drinnan CT, Geuss LR, Suggs LJ. Vascular differentiation of bone marrow stem cells is directed by a tunable three-dimensional matrix. Acta Biomater 2010;6:3395-403.

[561] Hung HS, Chen HC, Tsai CH, Lin SZ. Novel approach by nanobiomaterials in vascular tissue engineering. Cell Transplant 2011;20:63-70.

[562] Goh YF, Shakir I, Hussain R. Electrospun fibers for tissue engineering, drug delivery, and wound dressing. J Mater Sci 2013;48:3027-54.

[563] Zahedi P, Rezaeian I, Ranaei-Siadat SO, Jafari SH, Supaphol P. A review on wound dressings with an emphasis on electrospun nanofibrous polymeric bandages. Polym Adv Technol 2010;21:77-95.

[564] Singh AV, Aditi AS, Gade WN, Vats T, Lenardi C, Milani P. Nanomaterials: New Generation Therapeutics in Wound Healing and Tissue Repair. Curr Nanosci 2010;6:577-86.

[565] Abdelgawad AM, Hudson SM, Rojas OJ. Antimicrobial wound dressing nanofiber mats from multicomponent (chitosan/silver-NPs/polyvinyl alcohol) systems. Carbohydr Polym 2014;100:166-78.

[566] Huang Y, Zhong Z, Duan B, Zhang L, Yang Z, Wang Y, Ye Q. Novel fibers fabricated directly from chitin solution and their application as wound dressing. J Mater Chem B Mater Biol Med 2014;2:3427-32. 
[567] Shahverdi S, Hajimiri M, Esfandiari MA, Larijani B, Atyabi F, Rajabiani A, Dehpour AR, Gharehaghaji AA, Dinarvand R. Fabrication and structure analysis of poly (lactide-co-glycolic acid)/silk fibroin hybrid scaffold for wound dressing applications. Int J Pharm 2014;473:345-55.

[568] Lee CH, Shin HJ, Cho IH, Kang YM, Kim IA, Park KD, Shin JW. Nanofiber alignment and direction of mechanical strain affect the ECM production of human ACL fibroblast Biomaterials. 2005;26:1261-70.

[569] Dvir T, Timko BP, Brigham MD, Naik SR, Karajanagi SS, Levy O, Jin HW, Parker KK, Langer R, Kohane DS. Nanowired three-dimensional cardiac patches. Nat Nanotechnol 2011;6:720-5. 
Fig. 1. Polymeric nanostructured materials for biomedical applications.

Fig. 2. Schematic illustration of the preparation of the DOX-NPs. [59], Copyright 2013. Reproduced with permission from Elsevier Ltd.

Fig. 3. Schematic description of the preparation procedure and structure of polymersomes of asymmetrical bilayer membrane. Left diagram: preparation procedure by adding two block copolymers, $\mathrm{DEX}_{22}-\mathrm{PCL}_{66}$ and $\mathrm{PEG}_{45}-\mathrm{PCL}_{30}$, into a dextran/PEG aqueous two-phase system; right diagram: polymersome structure consisting of a dextran core in which bio-macromolecules are packed by preferential partition and an asymmetric block copolymer bilayer shell with the dextran block facing the core and PEG block facing the PEG continuous phase. [88], Copyright 2010. Reproduced with permission from Elsevier Ltd.

Fig. 4. Illustration of reversibly crosslinked temperature-responsive nano-sized polymersomes. (a) Formation of polymersomes by simply increasing temperature; (b) stabilization of the vesicular structure via crosslinking at the interface between the membrane and hydrophilic PEG layer; (c) formation of swollen polymersomes by decreasing temperature below its LCST; (d) destabilization of polymersomes by decreasing temperature below its LCST in the presence of $10 \mathrm{mM}$ 1,4-dithio-DL-threitol (DTT). [108], Copyright 2009. Reproduced with permission from the Royal Society of Chemistry.

Fig. 5. Synthesis of polyvalent propargyl ether nanopods. [161], Copyright (2010). Reprinted with permission from the American Chemical Society.

Fig. 6. Synthesis and stimuli-responsive properties of the nanogels. [183], Copyright 2011. Reproduced with permission from Elsevier Ltd.

Fig. 7. (A) Molecular structure of CPA1 that contains four segments: lipophilic, $\beta$ sheet, spacer, and epitope segments. A bioactive GFOGER sequence is inserted within the repeating structural unit GPO as the epitope segment. (B) CPA1 self-assembly process: three collagen-mimetic peptide head groups self- 
assemble into a triple helix, while the hydrophobic tails and $\beta$-sheet-type hydrogen bonding drive and guide the assembly of triple-helical CPA1 into nanofibers. The peptide portion is exposed on the periphery of the nanofiber. [255], Copyright (2011). Reprinted with permission from the American Chemical Society.

Fig. 8. Chemical structures of natural and synthetic polymers used for constructions of polymer-drug conjugates.

Fig. 9. Schematic diagram of the principles of gene therapy. Therapeutic genes of interest or growth factors that influence cellular function can be placed in viral or nonviral vectors that enter a targeted cell to significantly alter its function. [395], Copyright 2006. Reproduced with permission from Elsevier Ltd.

Fig. 10. (a) Schematic representation of the preparation of the cancer-recognizable MRI contrast agents (CR-CAs); Amphiphilic block copolymers (i.e., PEG-p(L-LA)DTPA-Gd and PEG-p(L-His) self-assemble into micelles in an aqueous solution at $\mathrm{pH}$ 7.4. (b) Schematic representation of $\mathrm{pH}$-dependent structural transformation and related MR signal change in CR-CAs. Inset: Chemical structural representation of the protonation of imidazole groups in PEG-p(LHis) at acidic $\mathrm{pH}$. (c) Schematic representation of the tumor-accumulation behavior of (1) conventional micelle-based CAs and (2) CR-CAs. [453], Copyright 2014. Reproduced with permission from Elsevier Ltd.

Fig. 11. Multifunctional nanoscale strategies, including scaffolding, imaging, and bioactive molecule delivery systems for vascular tissue engineering. [557], Copyright 2012. Reproduced with permission from Elsevier Ltd. 


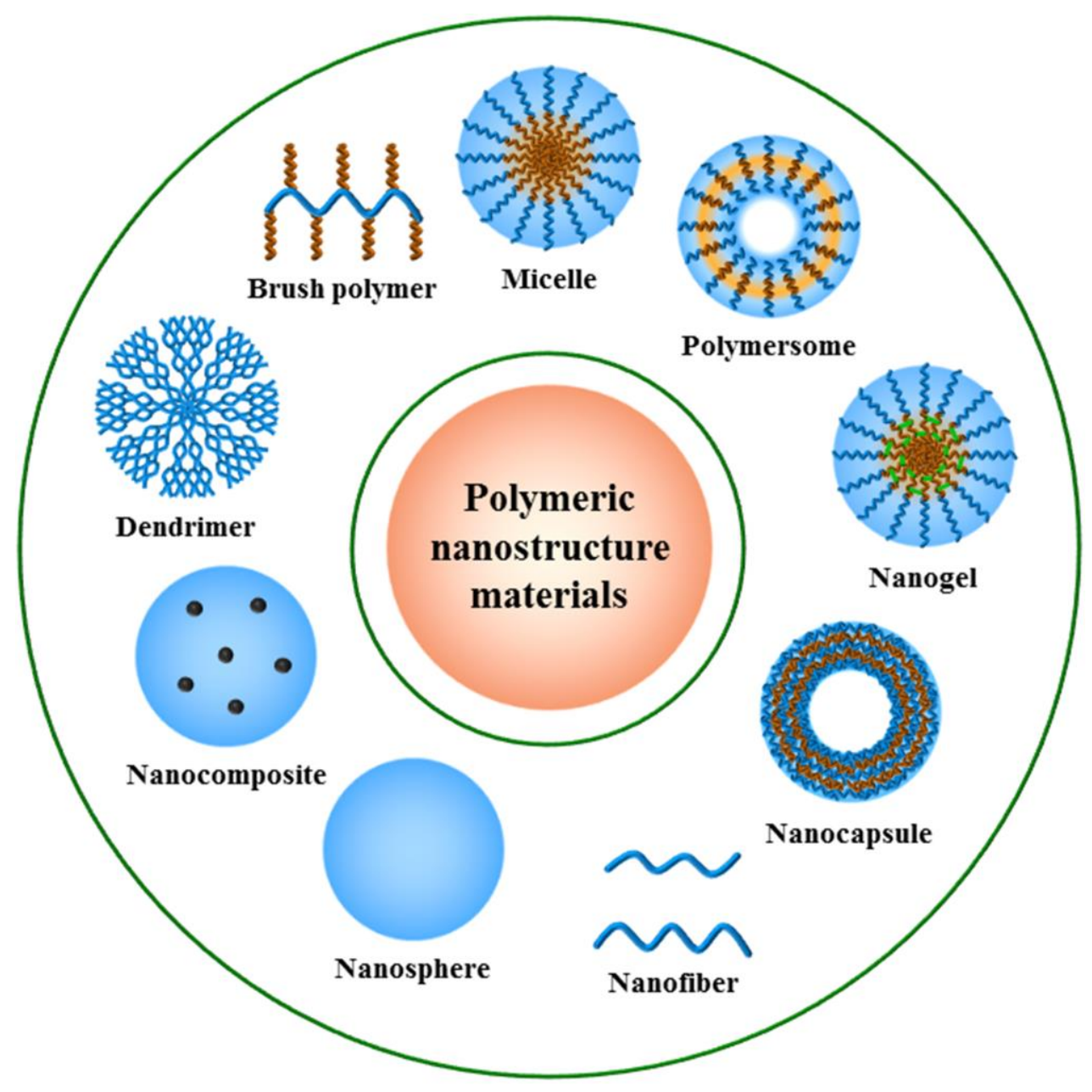

Figure 1 

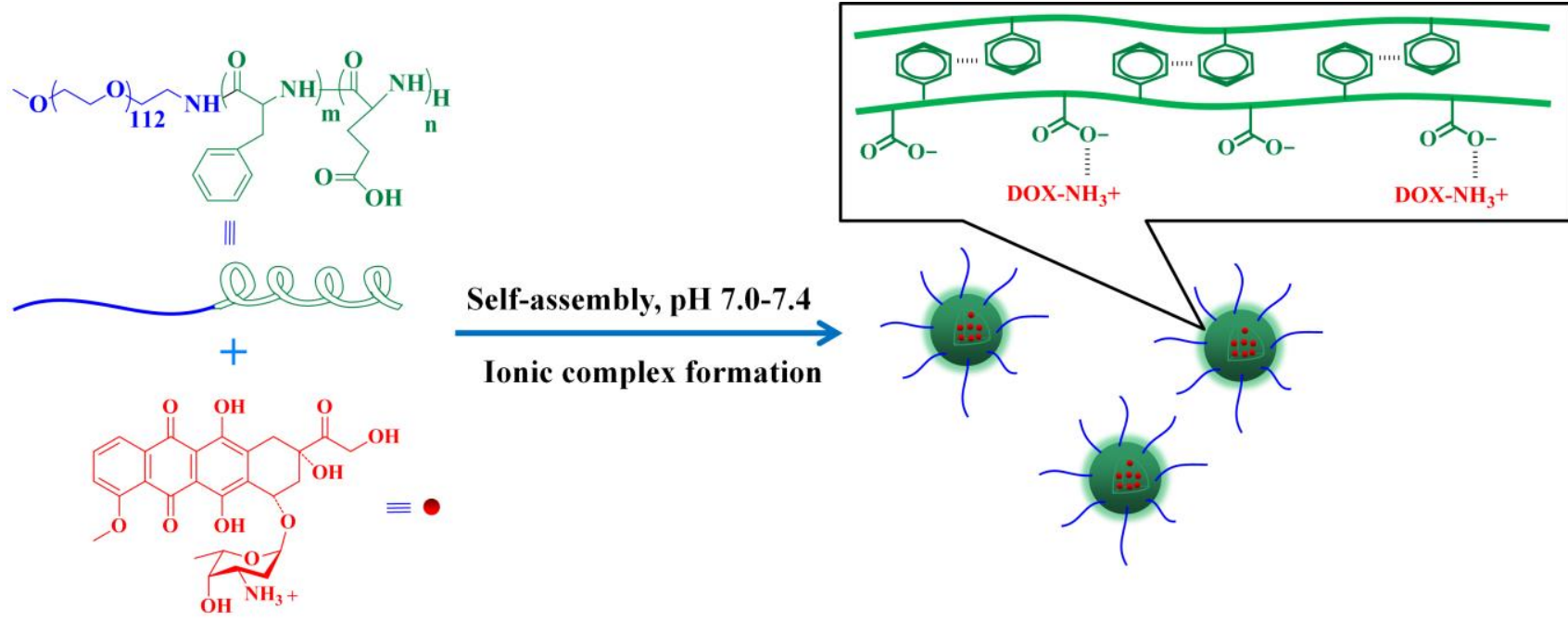

Self-assembly, pH 7.0-7.4

Ionic complex formation

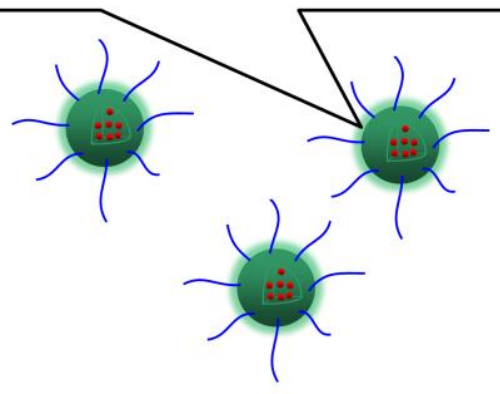

Figure 2 


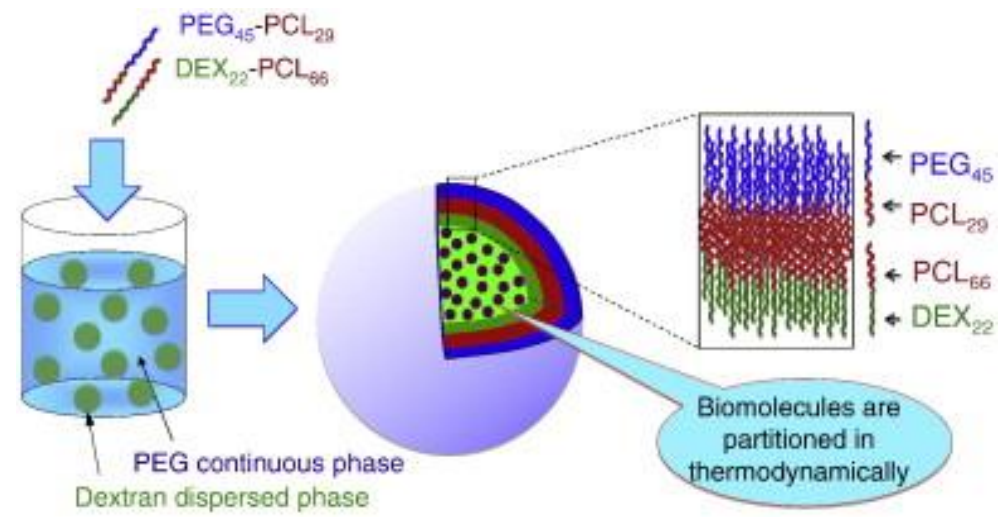

Figure 3 


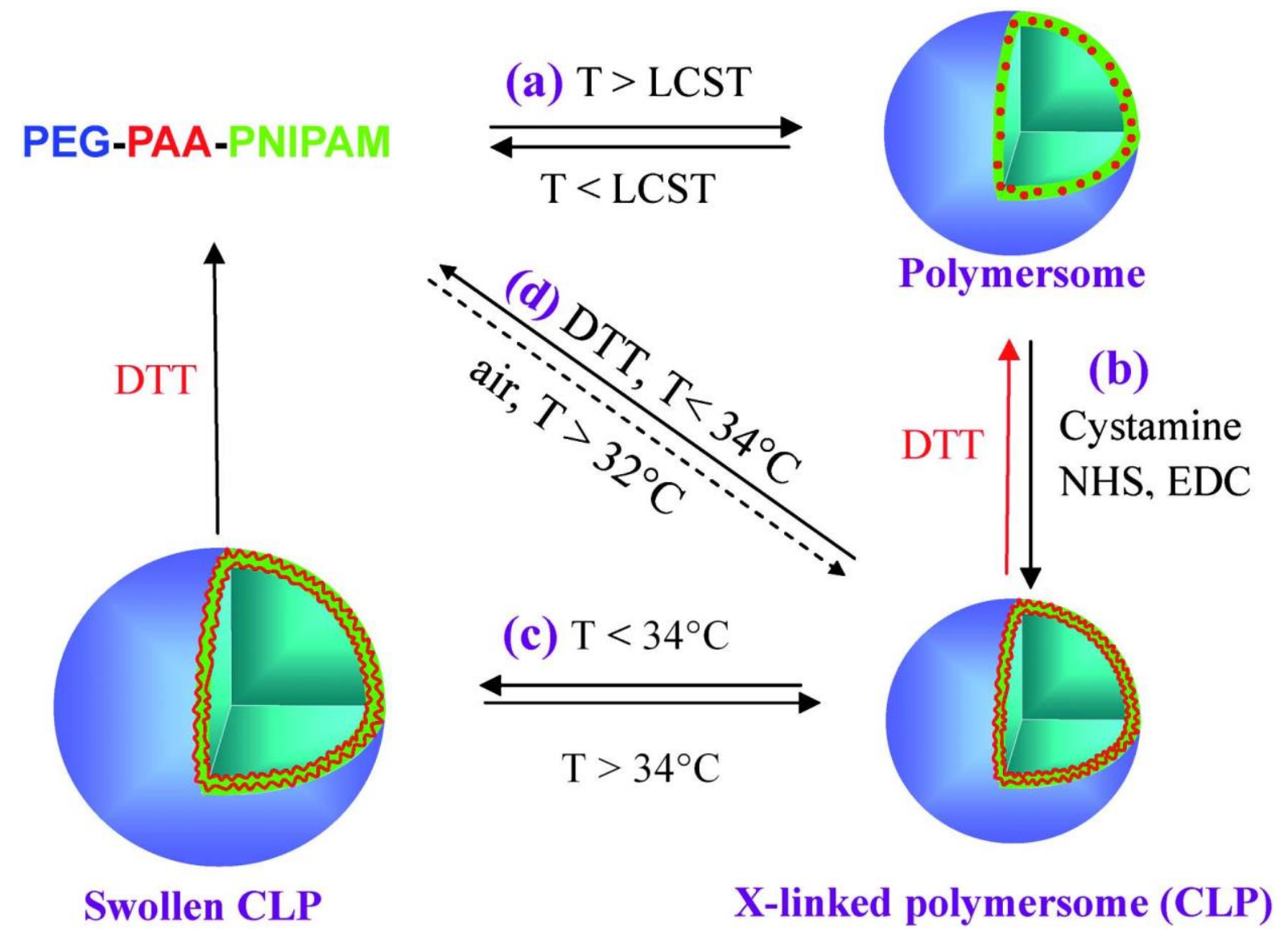

Figure 4 


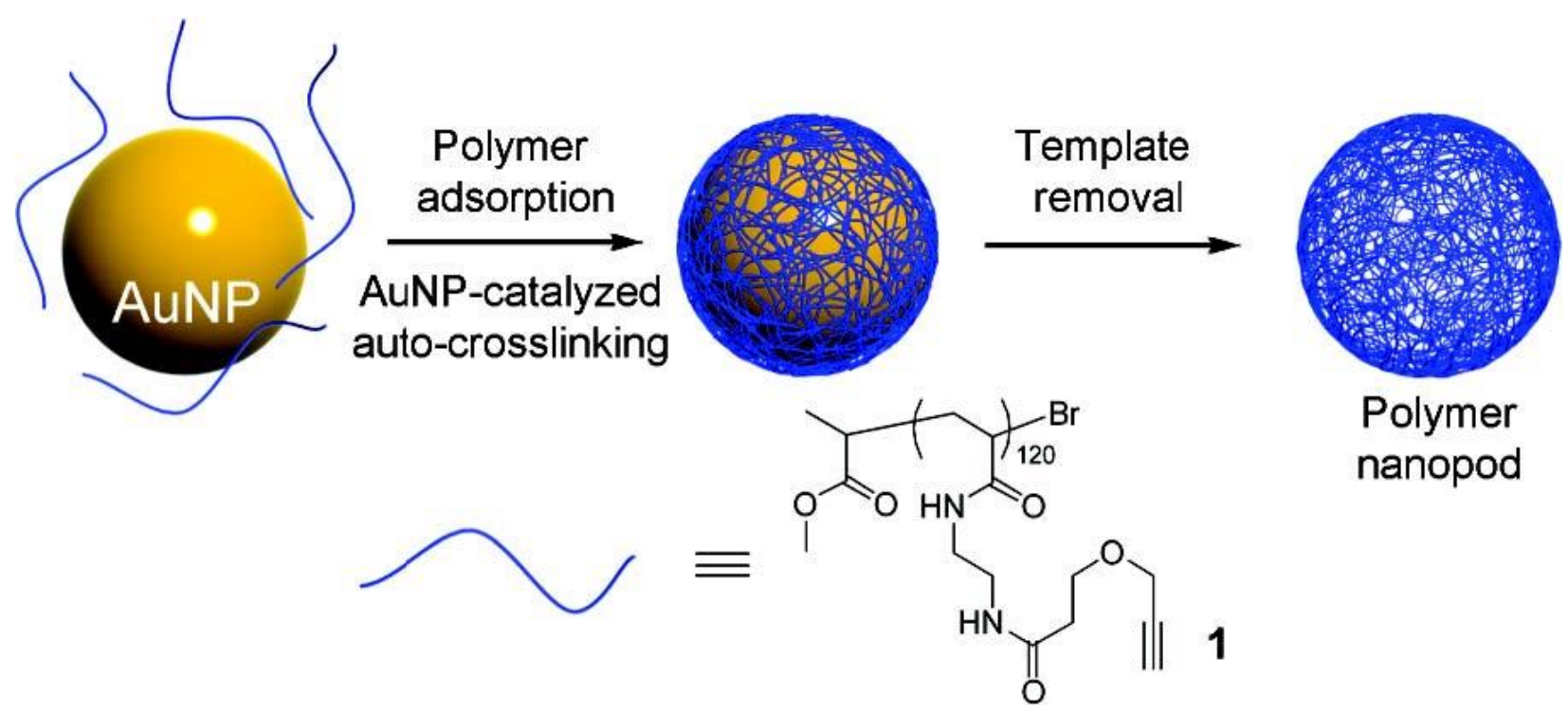

Figure 5 


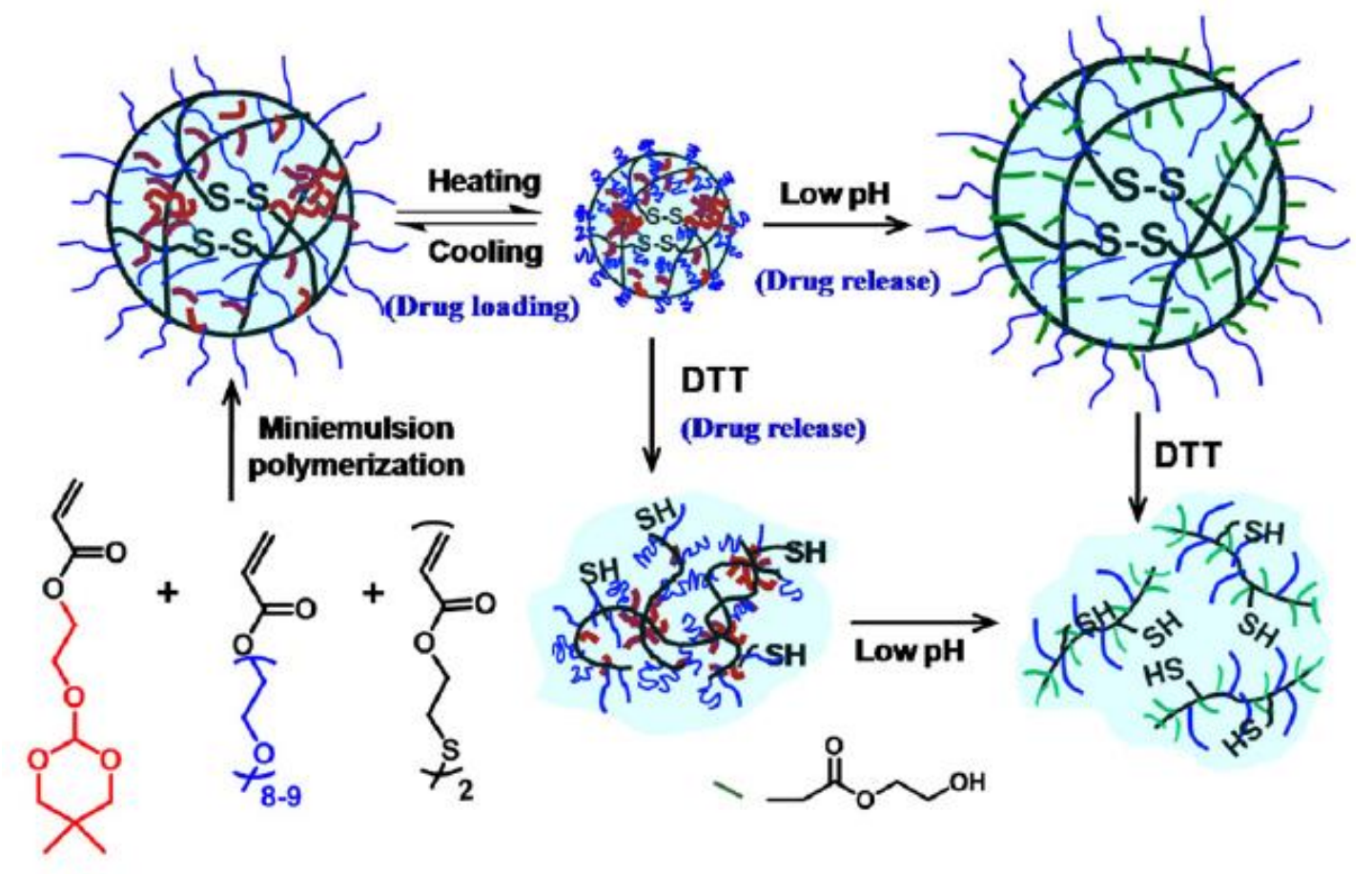

Figure 6 
(A)

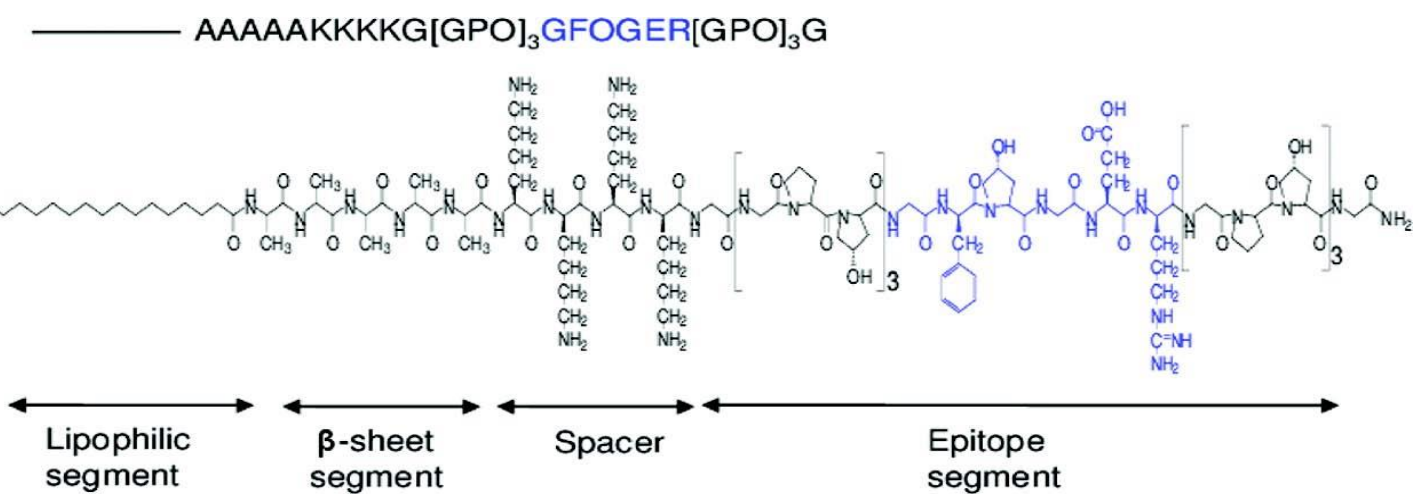

(B)
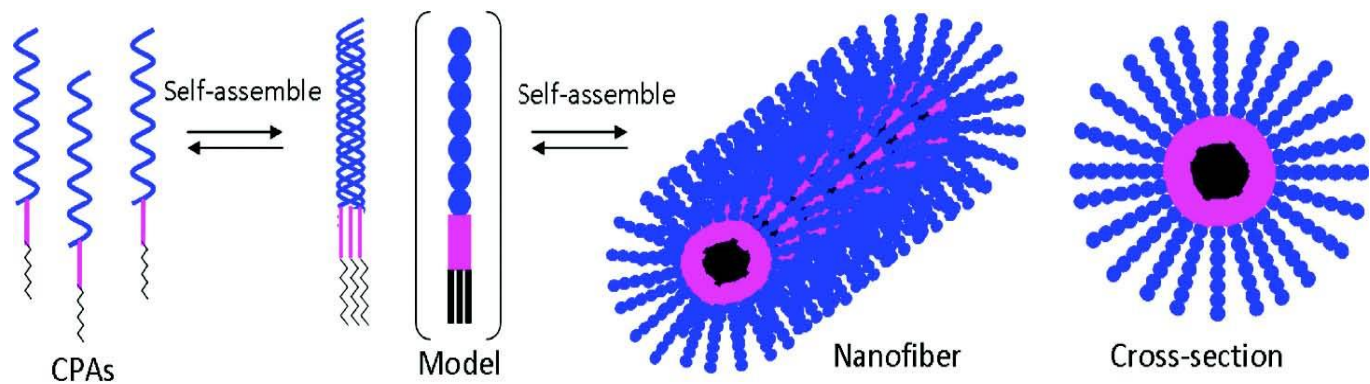

Figure 7 
A) Polysaccarides
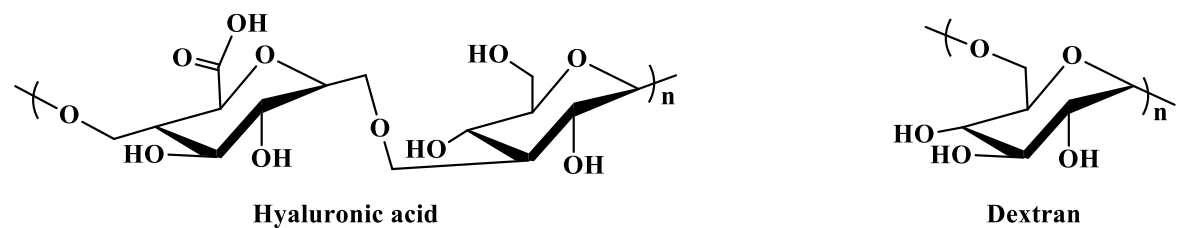

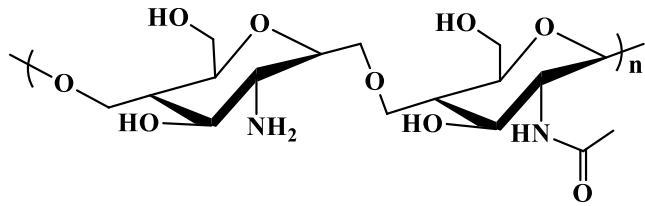

Chitosan

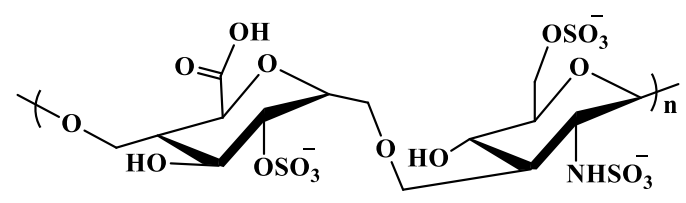

Heparin

B) Liner hydrophilic synthetic polymers<smiles>CC(O)CNC(=O)C1CC1CC1CC1</smiles>

HPMA (copolymer)

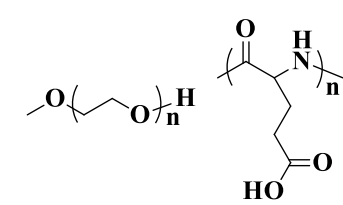

PEG

PGA<smiles>C=CC(CC(=O)O)NC(C)=O</smiles>

PAA

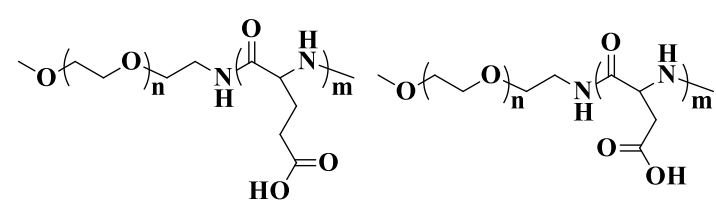

PEG-b-PGA PEG-b-PAA

C) Dendrimers

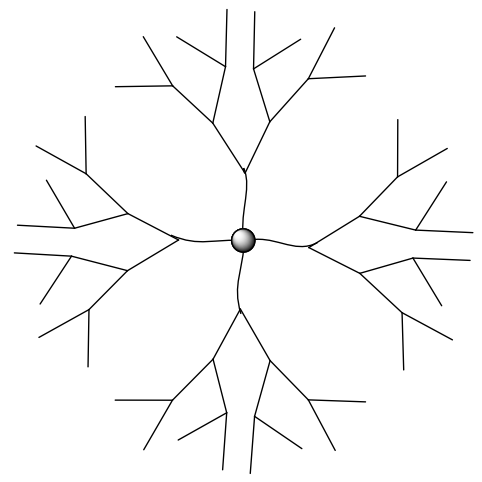

Dendrimer

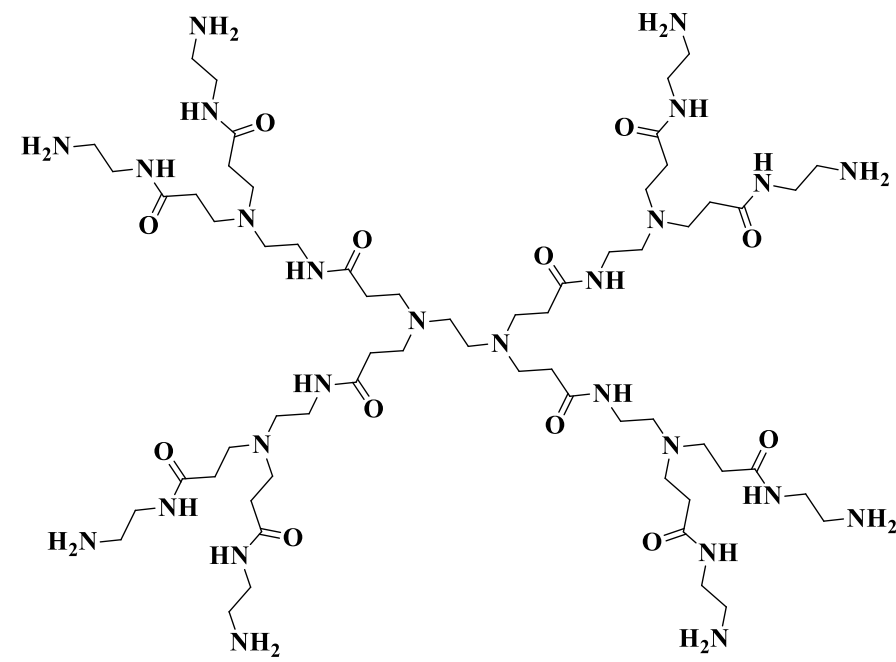

PAMAM

D) Amphiphilic block copolymers

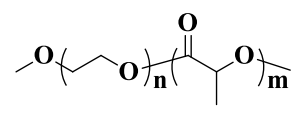

PEG-b-PLA

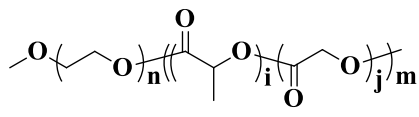

PEG- $b$-PLGA

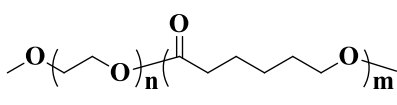

PEG-b-PCL

\section{Figure 8}




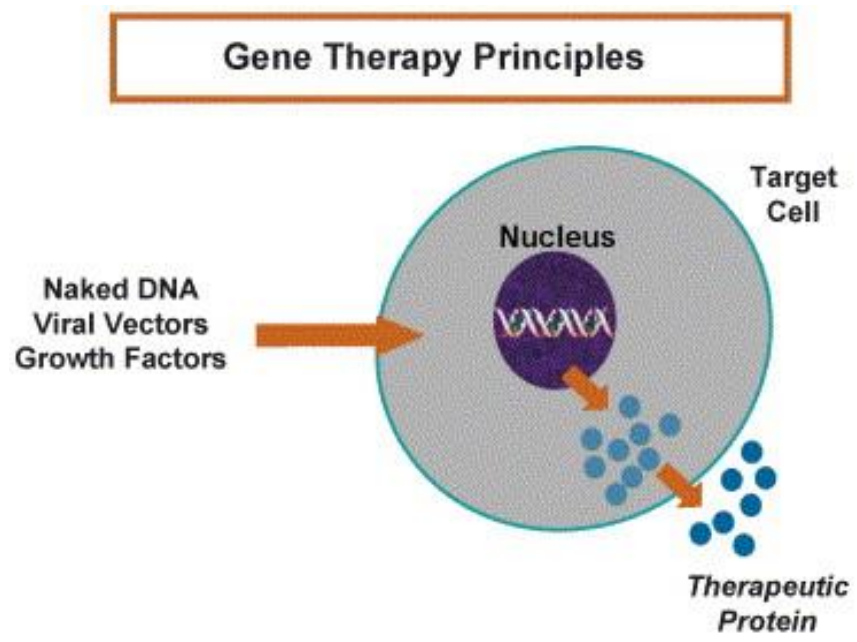

Figure 9 
a

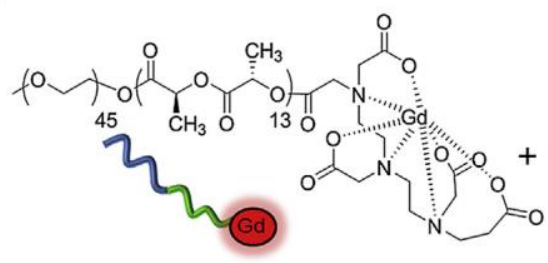

PEG-p(L-LA)-DTPA-Gd

b

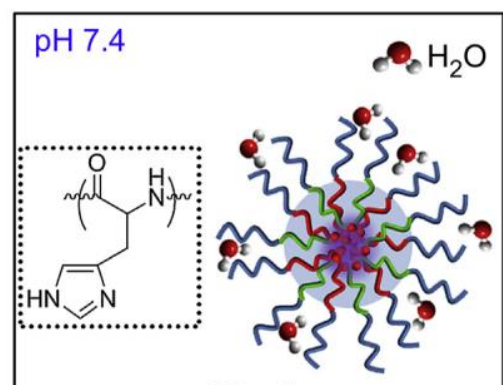

Micelle

Weak MR signal
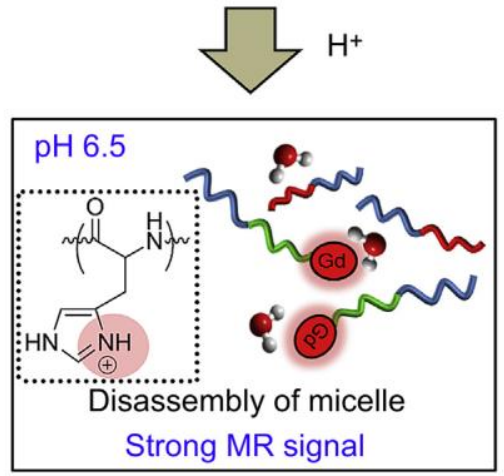

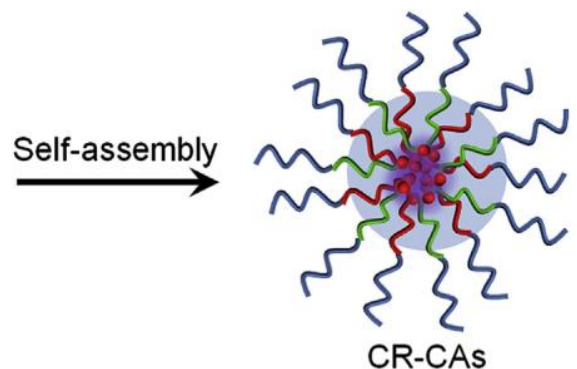

C

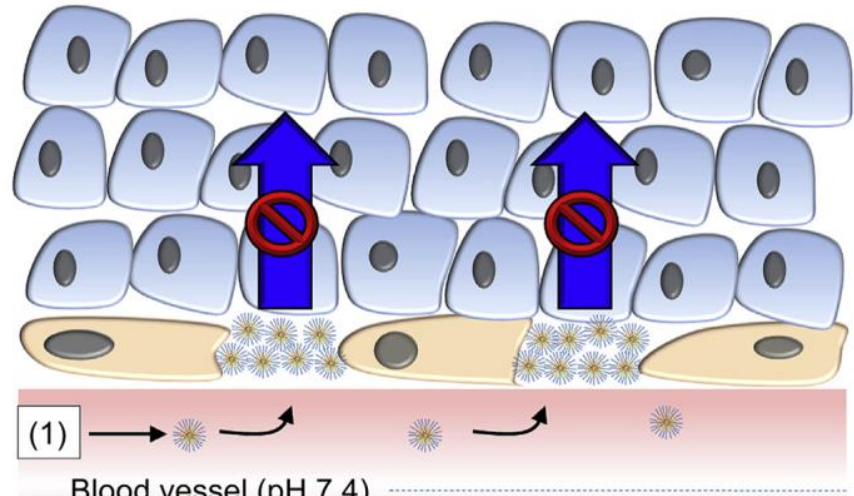

Blood vessel $(\mathrm{pH} 7.4)$

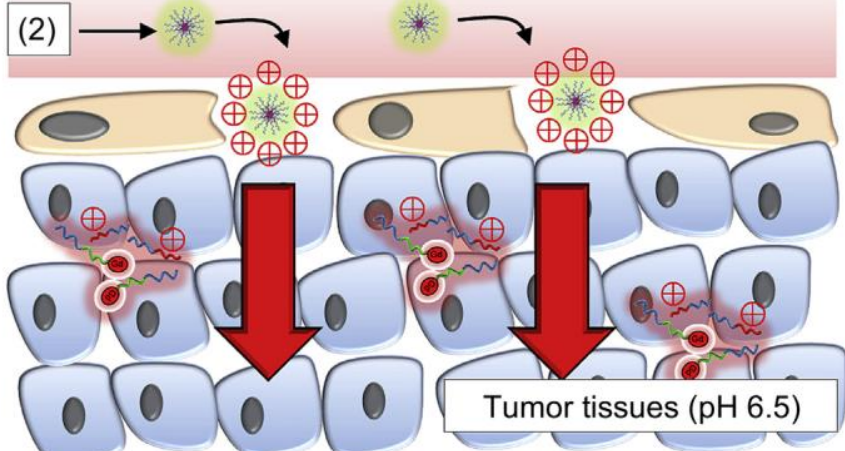

Figure 10 


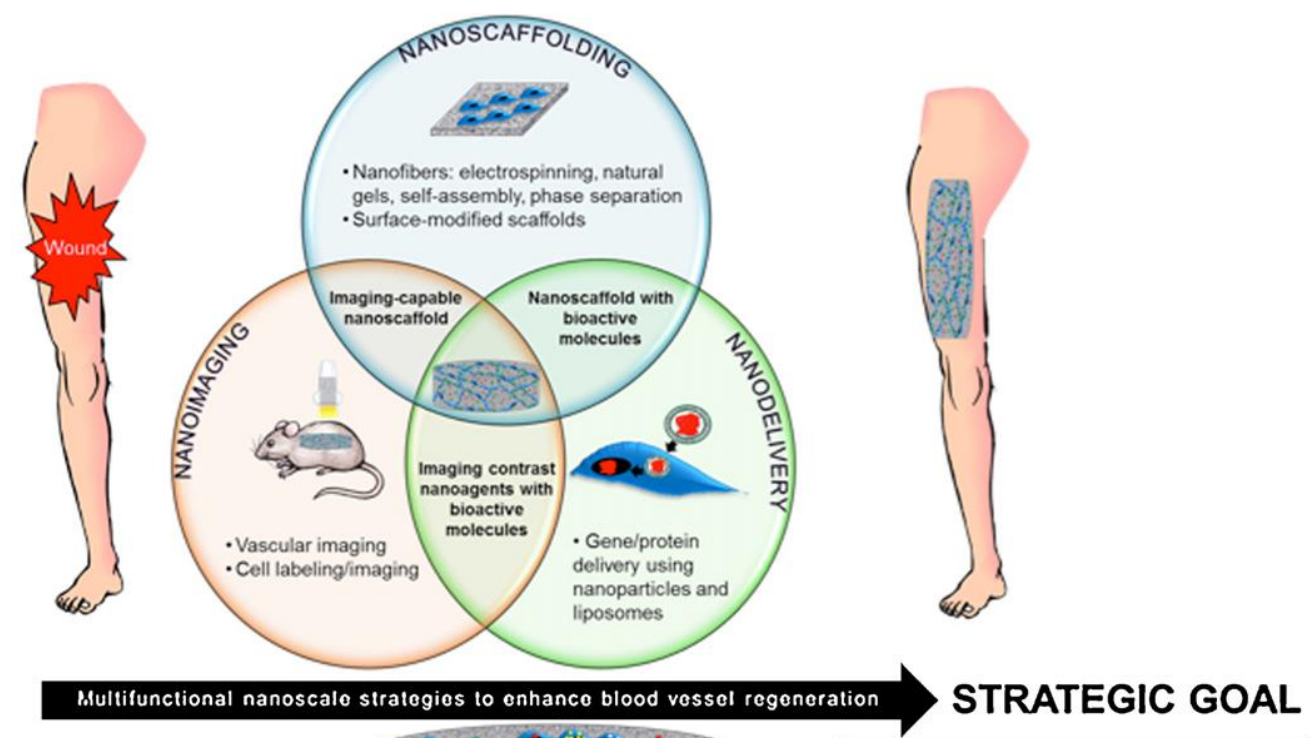

Seeded cell

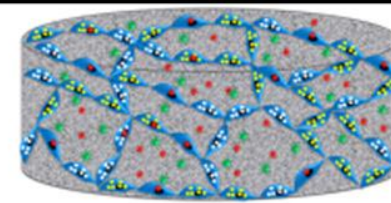

- Biomimetic environment for encapsulated cells

- Monitoring vascular regeneration

Gene nanodelivery system

Peptide/protein nanodelivery system

Imaging contrast nanoagent

Imaging contrast nanoagent with bioactive molecules

- Enhanced angiogenic protein production

Figure 11 
Table 1. Selective methods to prepare polymeric micelles with different compositions

\begin{tabular}{|c|c|c|c|}
\hline $\begin{array}{l}\text { Preparation } \\
\text { method }\end{array}$ & Composition & Driving force & Ref. \\
\hline \multirow{6}{*}{$\begin{array}{l}\text { Direct } \\
\text { dissolution }\end{array}$} & Pluronic block copolymer & Hydrophobic interaction & {$[42,43]$} \\
\hline & Poly(2-isopropyl-2-oxazoline)-b-poly(L-lysine); & Electrostatic interaction & [44] \\
\hline & Poly(2-isopropyl-2-oxazoline)- $b$-poly(aspartic acid) & & \\
\hline & Methoxy poly(ethylene glycol)-b-poly(L-glutamic acid); & Electrostatic interaction & {$[45]$} \\
\hline & Doxorubicin hydrochloride & & \\
\hline & Poly(ethylene glycol)-poly(glutamic acid) + cisplatin & Complexation & [46]. \\
\hline \multirow{6}{*}{$\begin{array}{l}\text { Film } \\
\text { casting }\end{array}$} & Methoxy poly(ethylene glycol)-block-poly( $\delta$-valerolactone $)$ & Hydrophobic interaction & [47] \\
\hline & Methoxy poly(ethylene glycol)-block-poly(D,L-lactide) & Hydrophobic interaction & {$[48]$} \\
\hline & $\begin{array}{l}\text { Acid-functionalized poly(carbonate) and poly(ethylene glycol) diblock } \\
\text { copolymer; }\end{array}$ & $\begin{array}{l}\text { Hydrophobic and electrostatic } \\
\text { interaction }\end{array}$ & [49] \\
\hline & $\begin{array}{l}\text { Urea-functionalized poly(carbonate) and poly(ethylene glycol) diblock } \\
\text { copolymer; }\end{array}$ & & \\
\hline & Thioridazine hydrochloride & & \\
\hline & $\begin{array}{l}\text { Methoxy poly(ethylene glycol)-block-decylamine-grafted poly(L-aspartic acid); } \\
\text { Cypate }\end{array}$ & Hydrophobic interaction & {$[50]$} \\
\hline \multirow[t]{3}{*}{ Dialysis } & Poly(ethylene oxide)-poly( $\beta$-benzyl L-aspartate) & Hydrophobic interaction & {$[51]$} \\
\hline & Poly(2-(4-vinylbenzyloxy)-N,N-diethylnicotinamide); & Hydrophobic interaction & {$[52]$} \\
\hline & Paclitaxel & & \\
\hline
\end{tabular}


Poly(ethylene oxide)- $b$-poly( $\varepsilon$-caprolactone)

PEG-dendritic polylysine-camptothecin

poly(ethylene glycol)- $b$-poly( $\varepsilon$-caprolactone);

Poly( $\varepsilon$-caprolactone)- $b$-poly(2-aminoethyl ethylene phosphate);

siRNA

Oil-in-water Poly(ethylene glycol)-block-poly(alkyl acrylate-co-methacrylic acid)s; emulsion

\section{Fenofibrate}

Poly(ethylene glycol)- $b$-poly(alkyl acrylate-co-t-butyl methacrylate);

Fenofibrate

Methoxy poly(ethylene glycol)-block-poly(D,L-lactide);

Poly(D,L-lactide);

Docetaxel
Hydrophobic interaction

Hydrophobic interaction

Hydrophobic and electrostatic

[55] interaction

Hydrophobic interaction

Hydrophobic interaction

Hydrophobic interaction 
Table 2. Selective polymer-drug conjugates under clinical trials

\begin{tabular}{|c|c|c|c|c|c|c|c|}
\hline Trade name & Excipient & LMW drug & $\begin{array}{l}\text { Diameter } \\
(\mathrm{nm})\end{array}$ & $\begin{array}{l}\text { Drug loading } \\
\text { content } \\
(\mathrm{wt} \%)\end{array}$ & Indications & Status & Ref. \\
\hline AP5280 & $\begin{array}{l}\text { HPMA } \\
\text { copolymer }\end{array}$ & $\begin{array}{l}\text { Carboplatin } \\
\text { palatinate } \\
\text { malonato-platinate }\end{array}$ & $-{ }^{\mathrm{a}}$ & $8.5(7)$ & Various cancers & $\begin{array}{l}\text { Phase I/II } \\
\text { (Netherlands) }\end{array}$ & [287] \\
\hline $\begin{array}{l}\text { MAG-CPT, } \\
\text { PNU166148 }\end{array}$ & $\begin{array}{l}\text { HPMA } \\
\text { copolymer }\end{array}$ & $\mathrm{CPT}$ & $-{ }^{\mathrm{a}}$ & 10 & Various cancers & $\begin{array}{l}\text { Phase I (UK, } \\
\text { discontinued) }\end{array}$ & $\begin{array}{l}{[288,} \\
289]\end{array}$ \\
\hline $\begin{array}{l}\text { AP5346, } \\
\text { ProLindac }^{\mathrm{TM}}\end{array}$ & $\begin{array}{l}\text { HPMA } \\
\text { copolymer }\end{array}$ & DACH platinate & $-{ }^{\mathrm{a}}$ & $-{ }^{\mathrm{a}}$ & $\begin{array}{l}\text { Various cancers, particularly } \\
\text { ovarian and colorectal cancers }\end{array}$ & Phase II (France) & [290] \\
\hline $\begin{array}{l}\text { PK1, } \\
\text { FCE28068 }\end{array}$ & $\begin{array}{l}\text { HPMA } \\
\text { copolymer }\end{array}$ & DOX & $-{ }^{\mathrm{a}}$ & 8.5 & $\begin{array}{l}\text { Various cancers, particularly } \\
\text { lung and breast cancer }\end{array}$ & Phase II (UK) & [291] \\
\hline $\begin{array}{l}\text { PK2, } \\
\text { FCE28069 }\end{array}$ & $\begin{array}{l}\text { HPMA } \\
\text { copolymer-galact } \\
\text { osamine }\end{array}$ & DOX & 8.4 & 7.5 & $\begin{array}{l}\text { Particularly hepatocellular } \\
\text { carcinoma }\end{array}$ & $\begin{array}{l}\text { Phase I/II (UK, } \\
\text { discontinued) }\end{array}$ & [292] \\
\hline PNU166945 & $\begin{array}{l}\text { HPMA } \\
\text { copolymer }\end{array}$ & PTX & $-{ }^{\mathrm{a}}$ & 5 & Various cancers & $\begin{array}{l}\text { Phase I } \\
\text { (Netherlands, } \\
\text { discontinued) }\end{array}$ & [293] \\
\hline $\begin{array}{l}\text { Pegamotecan, } \\
\text { Prothecan }^{\mathrm{TM}}\end{array}$ & PEG & $\mathrm{CPT}$ & $-^{\mathrm{a}}$ & 1.7 & Various cancers & $\begin{array}{l}\text { Phase II (USA } \\
\text { discontinued) }\end{array}$ & [294] \\
\hline NKTR-105 & PEG & DTX & $-^{\mathrm{a}}$ & $-^{\mathrm{a}}$ & Various cancers & Phase I (USA) & [295] \\
\hline NKTR-102 & PEG & Irinotecan & $-{ }^{\mathrm{a}}$ & $-{ }^{\mathrm{a}}$ & $\begin{array}{l}\text { Particularly ovarian and } \\
\text { colorectal cancers }\end{array}$ & Phase III (USA) & [296] \\
\hline
\end{tabular}




\begin{tabular}{|c|c|c|c|c|c|c|c|}
\hline $\begin{array}{l}\text { NKTR-118 } \\
\text { (oral) }\end{array}$ & PEG & Naloxone & $--^{a}$ & $-{ }^{\mathrm{a}}$ & Opioid-induced constipation & Phase II (USA) & [297] \\
\hline EZN-2208 & PEG & $\mathrm{SN}-38$ & $-{ }^{\mathrm{a}}$ & $-^{\mathrm{a}}$ & Various cancers & Phase I (USA) & [298] \\
\hline CT-2106 & PGA & СРТ & $-^{\mathrm{a}}$ & $33 \sim 35$ & $\begin{array}{l}\text { Various cancers, particularly } \\
\text { lung, ovarian and colorectal } \\
\text { cancers }\end{array}$ & Phase I/II (USA) & [299] \\
\hline $\begin{array}{l}\text { CT-2103, } \\
\text { Xyotax }^{\mathrm{TM}} \\
\text { Opaxio }^{\circledR}\end{array}$ & PGA & PTX & $-^{\mathrm{a}}$ & 37 & $\begin{array}{l}\text { Various cancers, particularly } \\
\text { NSCLC, ovarian cancer as a } \\
\text { single agent or in combination } \\
\text { therapy }\end{array}$ & Phase III (USA) & $\begin{array}{l}{[300,} \\
301]\end{array}$ \\
\hline DE-310 & $\begin{array}{l}\text { Carboxymethylde } \\
\text { xtran }\end{array}$ & Exatecan & $-^{\mathrm{a}}$ & 6.6 & Various cancers & $\begin{array}{l}\text { Phase I } \\
\text { (Netherlands) }\end{array}$ & [302] \\
\hline $\begin{array}{l}\text { AD-70, } \\
\text { DOX-OXD }\end{array}$ & Dextran & DOX & $-^{\mathrm{a}}$ & $-^{\mathrm{a}}$ & Various cancers & $\begin{array}{l}\text { Phase I } \\
\text { (Germany) }\end{array}$ & [303] \\
\hline $\begin{array}{l}\text { XMT-1001, } \\
\text { PHF-CPT }\end{array}$ & Polyacetal & CPT & $-{ }^{a}$ & $5 \sim 7$ & Various cancers & Phase I (USA) & $\begin{array}{l}{[304,} \\
305]\end{array}$ \\
\hline NK012 & PEG- $b$-PGA & $\mathrm{SN}-38$ & 20 & 20.0 & $\begin{array}{l}\text { Colorectal, advanced } \\
\text { metastatic triple negative } \\
\text { breast cancer, relapsed small } \\
\text { cell lung cancer and SCLC }\end{array}$ & $\begin{array}{l}\text { Phase II (Japan } \\
\text { and USA) }\end{array}$ & $\begin{array}{l}{[306,} \\
307]\end{array}$ \\
\hline NK911 & PEG- $b$-PAA & DOX & 40 & $-^{\mathrm{a}}$ & Metastatic pancreatic cancer & Phase II (Japan) & $\begin{array}{l}{[308,} \\
309]\end{array}$ \\
\hline
\end{tabular}

DACH, diaminocyclohexane; SN-38, 7-ethyl-10-hydroxycamptothecin; NSCLC, non-small cell lung cancer; SCLC, small cell lung cancer; ${ }^{a}$ Not available. 
Table 3. Selective polymeric micelle formulations for cancer therapeutics under clinical trials

\begin{tabular}{|c|c|c|c|c|c|c|c|c|}
\hline Trade name & Excipient & LMW drug & $\begin{array}{l}\text { Loading } \\
\text { mode }\end{array}$ & $\begin{array}{l}\text { Diamete } \\
\mathrm{r}(\mathrm{nm})\end{array}$ & $\begin{array}{l}\text { Drug loading } \\
\text { content } \\
\text { (wt.\%) }\end{array}$ & Indications & Status & Ref. \\
\hline SP1049C & $\begin{array}{l}\text { Pluronic }{ }^{\circledR} \text { L61, } \\
\text { F127 }\end{array}$ & DTX & $\begin{array}{l}\text { Physical } \\
\text { entrapme } \\
\text { nt }\end{array}$ & 30 & 8.2 & $\begin{array}{l}\text { Advanced gastric and } \\
\text { esophageal cancer }\end{array}$ & Phase II (Canada) & [359] \\
\hline $\begin{array}{l}\text { Genexol }^{\circledR}-\mathrm{P} \\
\mathrm{M}\end{array}$ & PEG- $b$-PDLLA & PTX & $\begin{array}{l}\text { Physical } \\
\text { entrapme } \\
\text { nt }\end{array}$ & $<50$ & 16.7 & $\begin{array}{l}\text { NSCLC, ovarian, breast and } \\
\text { gastric cancers }\end{array}$ & $\begin{array}{l}\text { Approved (Korea) and } \\
\text { Phase II (USA and } \\
\text { Russia) }\end{array}$ & $\begin{array}{l}{[357,} \\
358]\end{array}$ \\
\hline BIND-014 & $\begin{array}{l}\text { PEG- } b \text {-PDLLA } \\
\text { or } \\
\text { PEG- } b \text {-PLGA }\end{array}$ & DTX & $\begin{array}{l}\text { Physical } \\
\text { entrapme } \\
\text { nt }\end{array}$ & 100 & 10 & $\begin{array}{l}\text { Advanced or metastatic } \\
\text { solid cancers }\end{array}$ & Phase II (USA) & {$[360]$} \\
\hline NC-4016 & PEG- $b$-PGA & Oxaliplatin & $\begin{array}{l}\text { Coordinat } \\
\text { e bonding }\end{array}$ & 40 & $-^{\mathrm{a}}$ & Various solid cancers & Phase I (Japan) & {$[361]$} \\
\hline NC-6004 & PEG- $b$-PGA & Cisplatin & $\begin{array}{l}\text { Coordinat } \\
\text { e bonding }\end{array}$ & 30 & 39.0 & Advance solid cancers & Phase II/III (East Asia) & {$[362]$} \\
\hline NK105 & PEG- $b$-PPBA & PTX & $\begin{array}{l}\text { Physical } \\
\text { entrapme } \\
\text { nt }\end{array}$ & 85 & 23.0 & Advanced stomach cancer & Phase III (Japan) & {$[363]$} \\
\hline
\end{tabular}

PPBA, polyaspartate modified with 4-phenyl-butanol; ${ }^{\text {a }}$ Not available. 
Table 4. Selective polymer-peptide/protein conjugates in the market or under clinical trials

\begin{tabular}{|c|c|c|c|c|c|c|}
\hline Trade name & Excipient & Peptide/Protein & Administration & Indications & Status & Ref. \\
\hline Adagen $^{\circledR}$ & PEG & Adenosine deaminase & Intramuscular injection & $\begin{array}{l}\text { Severe combined immunodeficiency } \\
\text { disease }\end{array}$ & Market & [381] \\
\hline Oncaspar $^{\circledR}$ & PEG & L-asparaginase & Intramuscular injection & Acute lymphoblastic leukemia & Market & [382] \\
\hline Neulasta $^{\mathrm{TM}}$ & PEG & $\mathrm{G}-\mathrm{CSF}$ & Subcutaneous injection & Neutropenia & Market & [383] \\
\hline PEG-Asys ${ }^{\circledR}$ & PEG & Interferon $\alpha-2 \mathrm{a}$ & Subcutaneous injection & Hepatitis B and C & Market & [384] \\
\hline \multirow{3}{*}{ PEG-Intron ${ }^{\mathrm{TM}}$} & \multirow{3}{*}{ PEG } & \multirow{3}{*}{ Interferon $\alpha-2 b$} & \multirow{3}{*}{ Subcutaneous injection } & $\begin{array}{l}\text { Melanoma, chronic myeloid } \\
\text { leukaemia and renal-cell carcinoma }\end{array}$ & Phase I/II & [385] \\
\hline & & & & Hepatitis C & Market & [384] \\
\hline & & & & $\begin{array}{l}\text { Melanoma, multiple myeloma and } \\
\text { renal-cell carcinoma }\end{array}$ & Phase I/II & [385] \\
\hline Somavert $^{\circledR}$ & PEG & HGH antagonist & Intraperitoneal injections & Acromegaly & Market & [386] \\
\hline $\begin{array}{l}\text { Cimzia }^{\circledR}, \\
\text { CD870 }\end{array}$ & PEG & Anti-TNF- $\alpha-F a b$ & Subcutaneous injection & Crohn's disease, arthritis & Market & [387] \\
\hline Pegvisomant & PEG & $\begin{array}{l}\text { Human growth } \\
\text { hormone }\end{array}$ & Subcutaneous injection & Acromegaly & Market & [388] \\
\hline Hemospan $^{\circledR}$ & PEG & Hemoglobin & Intravenous infusions & $\begin{array}{l}\text { Delivery of } \mathrm{CO} \text { and } \mathrm{O}_{2} \text { in trauma } \\
\text { patients }\end{array}$ & $\begin{array}{l}\text { Phase II } \\
\text { (Swedish) }\end{array}$ & [389] \\
\hline ADI-PEG20 & PEG & Arginine deiminase & Intramuscular injection & Hepatocellular carcinoma & Phase II & [390] \\
\hline $\begin{array}{l}\text { PEG-PGA } \\
\text { and DON }\end{array}$ & PEG & $\begin{array}{l}\text { Glutaminase combined } \\
\text { with DON }\end{array}$ & Intravenous infusions & Various cancers & $\begin{array}{l}\text { Phase IIA } \\
\text { (Germany) }\end{array}$ & [391] \\
\hline $\begin{array}{l}\text { Zinostatin } \\
\text { Stimalamer }\end{array}$ & $\begin{array}{l}\text { Styrene maleic } \\
\text { anhydride }\end{array}$ & Neocarzinostatin & Intra-arterial infusions & Hepatocellular carcinoma & Market & [392] \\
\hline
\end{tabular}


G-CSF, granulocyte colony-stimulating factor; Anti-TNF- $\alpha-F a b$, antibody fragments against tumor necrosis factor $\alpha$; DON, 6-diazo-5-oxo-L-norleucine. ${ }^{a}$ Not available 